\title{
Developmental electrophysiological studies of letter - speech sound processing in normal reading and dyslexia
}

Citation for published version (APA):

Froyen, D. J. W. (2009). Developmental electrophysiological studies of letter - speech sound processing in normal reading and dyslexia. [Doctoral Thesis, Maastricht University]. Datawyse / Universitaire Pers Maastricht. https://doi.org/10.26481/dis.20091112df

Document status and date:

Published: 01/01/2009

DOI:

10.26481/dis.20091112df

Document Version:

Publisher's PDF, also known as Version of record

Please check the document version of this publication:

- A submitted manuscript is the version of the article upon submission and before peer-review. There can be important differences between the submitted version and the official published version of record.

People interested in the research are advised to contact the author for the final version of the publication, or visit the DOI to the publisher's website.

- The final author version and the galley proof are versions of the publication after peer review.

- The final published version features the final layout of the paper including the volume, issue and page numbers.

Link to publication

\footnotetext{
General rights rights.

- You may freely distribute the URL identifying the publication in the public portal. please follow below link for the End User Agreement:

www.umlib.nl/taverne-license

Take down policy

If you believe that this document breaches copyright please contact us at:

repository@maastrichtuniversity.nl

providing details and we will investigate your claim.
}

Copyright and moral rights for the publications made accessible in the public portal are retained by the authors and/or other copyright owners and it is a condition of accessing publications that users recognise and abide by the legal requirements associated with these

- Users may download and print one copy of any publication from the public portal for the purpose of private study or research.

- You may not further distribute the material or use it for any profit-making activity or commercial gain

If the publication is distributed under the terms of Article $25 \mathrm{fa}$ of the Dutch Copyright Act, indicated by the "Taverne" license above, 
Developmental electrophysiological studies of letter - speech sound processing in normal reading and dyslexia

Dries Froyen 
(C) Dries Froyen, Maastricht 2009

ISBN 978905278 XXX X

Universitaire Pers Maastricht 


\title{
Developmental electrophysiological studies of letter - speech sound processing in normal reading and dyslexia
}

\author{
Proefschrift
}

Ter verkrijging van de graad van doctor aan de Universiteit Maastricht, op gezag van de Rector Magnificus, Prof. mr. G. P. M. F. Mols volgens het besluit van het College van Decanen, in het openbaar te verdedigen op donderdag 12 november 2009 om 14.00 uur

$$
\text { door }
$$

\section{Dries Froyen}

geboren op 4 april 1980 te Genk 


\section{Promotor}

Prof. dr. L. Blomert

\section{Beoordelingscommissie}

Prof. dr. B. Jansma (voorzitter)

Prof. dr. V. Csépe (Hungarian Academy of Sciences, Budapest)

Prof. dr. R. Goebel

Prof. dr. B. McCandliss (Vanderbilt University, Nashville) 


\section{Table of contents}

$\begin{array}{lll}\text { Chapter } 1 & \text { General introduction }\end{array}$

Chapter 2 Cross-modal enhancement of the MMN to speech sounds indicates early and automatic integration of letters and speech sounds

Chapter 3 The Long road to automation: Neurocognitive development of letter - speech sound processing

Chapter $4 \quad$ Evidence for a specific cross-modal association deficit in dyslexia: An MMN-study of letter - speech sound processing

Chapter $5 \quad$ Exploring the role of low level visual processing in letter - speech sound integration: a visual MMN study

Chapter $6 \quad$ Letter-specificity of previously reported cross-modal effects on MMN to speech sounds 

Chapter 1

\section{General introduction}


The importance of written language in today's society can hardly be overestimated. It allows us to transcend our communication limits in space and time. Once something is written down, it can be preserved, copied and distributed. This makes it possible to acquire large amounts of knowledge at a high speed, and thus to learn from others' successes and failures. Consequently, our knowledge of the world has rapidly increased and our society has changed dramatically since literacy was taught worldwide. Nowadays, proficient literacy has become an essential prerequisite to obtain social and economic success in our knowledge-technology based society.

A crucial phase during the development towards successful acquisition of reading and writing skills is learning the associations between letters and speech sounds (Ehri, 2005; Frith, 1985; Marsh, Friedman, Welch, \& Desberg, 1981), i.e. the smallest units of respectively written and spoken language. Moreover, it is suggested that difficulties with associating letters and speech sounds are a cause of difficulties in learning to read in developmental dyslexia (Snowling, 1980; Vellutino, Fletcher, Snowling, \& Scanlon, 2004). Despite the assumed crucial role of letter - speech sound associations during learning to read, our understanding of single letter speech sound processing is still limited. The main aim of this thesis is to contribute to our understanding of letter - speech sound processing by investigating the automaticity and timing aspects of letter - speech sound processing in adult readers, during development and in children with dyslexia.

The first part of the introduction will focus on a neural model for letter - speech sound processing in literate adults (Van Atteveldt, Formisano, Goebel, \& Blomert, 2004). This model served as a starting point for the research presented in this thesis. Besides fully developed reading, typical reading development will be discussed which emphasizes the acquisition of letter - speech sound associations as a crucial step in reading acquisition (Ehri, 2005; Frith, 1985; Marsh et al., 1981). Although these associations are learned within months of reading instruction, recent behavioral reaction time data indicate protracted development of letter - speech sound processing (Blomert \& Vaessen, 2009). Declarative knowledge, i.e. knowing which letter goes with which speech sound, seems not the same as the automatic processing and integration of letters and speech sounds, presumably necessary for fluent reading development. Next to typical reading, developmental dyslexia is introduced. That is because, according to the phonological deficit theory of dyslexia, deficient letter - speech sound associations are considered to be the link between often observed phonological deficits and difficulties with learning to read (Snowling, 1980; Vellutino et al., 2004). However, direct experimental evidence for this assumption is currently lacking.

The last part of the introduction focuses on the methods applied in this thesis. The auditory mismatch negativity (aMMN), a well established ERP-component (Näätänen, 2000; Picton, Alain, Otten, Ritter, \& Achim, 2000), appeared a suitable tool to investigate the timing and automaticity of letter influences on speech sound 
processing. Finally, the visual counterpart of the aMMN, the visual mismatch negativity or vMMN (Czigler, 2007; Pazo-Alvarez, Cadaveira, \& Amenedo, 2003), will be discussed, since in one study the influence of speech sounds on letter processing is investigated with the use of the vMMN.

\section{Neural model for letter - speech sound processing:}

In an alphabetic writing system, single speech sounds are visually represented by written symbols, i.e. letters. Although some speech sounds are represented by more than one letter, e.g. the sound / $\mathrm{u}$ / is represented in Dutch by a compound of the two letters "o" and "e", most speech sounds represent one letter and vice versa. In this thesis the concept "letter" will be used to refer to a single letter representing a single speech sound, and the concept "speech sound" will be used to refer to a single speech sound representing a single letter.

Single letter - speech sound processing became only recently a frequent subject of scientific study. Multiple factors might have contributed to the previous lack of interest. The associations between letters and speech sounds are learned relatively easy and fast. Even in deep orthographies in which the relation between letters and their sounds is less consistent, a large number of letter - speech sound relations are learned within a year of reading instruction (Hardy, Smythe, Stennet, \& Wilson, 1972; Siegel \& Faux, 1989; Snowling, 1980). Aside from that, the process of coupling single letters with their respective speech sounds appears far from the actual reading process, making it seem less relevant for our understanding of reading, reading deficiencies and reading acquisition. The dominant reading theory, the Dual Route theory considers letter-speech sound processing only relevant for reading nonwords, but not for fluent word reading (Coltheart, Rastle, Perry, Langdon, \& Ziegler, 2001).

Nonetheless, since the first indications for real integration of letters with speech sounds (Dijkstra, Schreuder, \& Frauenfelder, 1989), the number of studies that have directly investigated letter - speech sound processing rapidly increased. An MEG study revealed the involvement of multisensory superior temporal areas (STS/STG) during letter - speech sound processing (Raij et al., 2000), These results were supported by the results from several fMRI studies (Blau et al., 2008; Hashimoto \& Sakai, 2004; Herdman et al., 2006; Van Atteveldt, Formisano, Blomert et al., 2007; Van Atteveldt et al., 2004; Van Atteveldt, Formisano, Goebel et al., 2007). Additionally, Van Atteveldt and coauthors (2004) showed that not only heteromodal areas in STS, but also early auditory areas (Heschl's sulcus and Planum Temporale) are involved in letter - speech sound integration. Congruent letter - speech sound pairs activated heteromodal as well as early auditory areas more in comparison with incongruent pairs. An effect of congruency reflects a content related link defined by previous experience between otherwise arbitrary symbols and speech sounds. Con- 
sequently, a congruency effect indicates real integration. Based on these results the following neural mechanism for letter - speech sound processing was proposed: Sensory specific input in early sensory areas and letter - speech sound integration in STS, followed by feedback to "sensory specific" auditory areas modulating speech sound processing depending on the congruency of the letter-speech sound combinations (Van Atteveldt et al., 2004).

Interestingly, however, whereas van Atteveldt and coauthors (2004; 2007a; 2007b) did not find congruency effects in low level visual areas, two studies reported the involvement of low level visual areas during letter - speech sound processing (Blau et al., 2008; Herdman et al., 2006). This discrepancy and eventual consequences for the current neural model of letter - speech sound processing will be discussed in chapter 5, in which the involvement of low level visual processing during letter speech sound processing is investigated using the visual MMN.

The previously discussed studies provided new insights in the network of brain areas involved in letter - speech sound processing. Until now, however, far less is known about its timing and automaticity. Two magnetoencephalography (MEG) studies provided high temporal resolution information, indicating letter - speech sound integration effects within 250 to 300 ms after stimulus onset (Herdman et al., 2006; Raij et al., 2000). However, in these MEG studies a stimulus related task was included to ensure participants constant focus on the stimuli. In a recent fMRI study, it was found that neural correlates of automatic letter - speech sound processing were overruled by task effects; low level auditory cortex activation, present during a passive condition, was not present when subjects were required to judge the congruency of letter - speech sound pairs (Van Atteveldt, Formisano, Goebel et al., 2007). This indicates that the neural network involved during an active letter speech sound task, might differ from passive/automatic letter - speech sound processing which is addressed in a natural reading or writing context. In chapter $\mathbf{2}$, a cross-modal MMN paradigm is proposed to investigate neural correlates of letter speech sound processing with high temporal resolution and without the requirement of attention. The validity of this cross-modal MMN paradigm for investigating letter - speech sound processing was shown in a study presented in chapter 6 .

\section{Development of letter - speech sound processing}

While spoken language is acquired spontaneously from birth, learning written language requires explicit instruction. That might be because the relation between letters and speech sounds is basically arbitrary in nature and merely based on a culturally defined consensus, which stands in contrast with the natural association between auditory and visual information in for example audiovisual speech (Calvert, Brammer, \& Iversen, 1998). In most countries written language starts to be explicitly instructed when children are six years old, by teaching the correspondences be- 
tween the letters of the alphabet and their respective speech sounds. It is generally accepted that learning the associations between letters and speech sounds is crucial to develop fluent reading at a later stage (Ehri, 2005; Frith, 1985; Marsh et al., 1981).

Most models of learning to read describe a developmental sequence in which the child develops from pre-alphabetic reading over alphabetic reading towards fluent reading by entire words or chunks of words (Ehri, 2005; Frith, 1985; Marsh et al., 1981). Only when the associations between letters and speech sounds are fully mastered, one can read unfamiliar words. Gradually, when words are read sufficiently frequently, they become accessible trough a direct and thus faster word recognition route. Although fluent word reading takes years to develop, mastery of letter - speech sound associations is assumed to be accomplished within a few years of reading instruction (Hardy et al., 1972; Siegel \& Faux, 1989; Snowling, 1980). In a relatively transparent language like Dutch, in which the letter - speech sound associations are mostly unambiguous, complete mastery of letter - speech sound associations is accomplished within one year of reading instruction (Aarnoutse, van Leeuwe, \& Verhoeven, 2000; Blomert \& Vaessen, 2009).

Recent data, however, indicate that knowing the associations between letters and speech sounds might not be the same as the instrumental use of this association during reading. In a study on a large nationwide sample of Dutch primary school children from six to twelve years old, not only accuracy but also reaction time data on letter - speech sound association were collected (Blomert \& Vaessen, 2009). Reaction times of letter - speech sound discrimination decisions steadily decreased during the whole range of Dutch primary school reading instruction. Since faster processing is one of the main criteria for the automaticity of a process (Schneider \& Chein, 2003), the extended decrease of processing time for letter - speech sound discrimination might indicate an extended development towards automatic letter speech sound association. While early acquisition of letter - speech sound associations is generally assumed in transparent languages, exactly when and how these associations develop in the human brain remains an open question, and is the topic of chapter 3.

\section{Letter - speech sound processing in dyslexia}

Most people acquire literacy skills without many difficulties. About ten percent of the Dutch population encounters difficulties with learning to read and/or write (Blomert, 2005). In most cases this is due to more general causes like overal learning problems or a combination of ineffective education and socio-economic problems. But about four percent of the total population (Blomert, 2005), suffers from literacy problems that cannot be explained by any of these factors and are diagnosed with developmental dyslexia. Developmental dyslexia is defined as a specific learning 
disability, characterized by reading and/or writing skills that are below what is predicted from their age and cognitive abilities (Lyon, Shaywitz, \& Shaywitz, 2003) The underlying cause of developmental dyslexia, from now on called dyslexia, is still under debate (Castles \& Coltheart, 2004; Ziegler \& Goswami, 2005).

The most commonly accepted hypothesis about dyslexia proposes a phonological deficit in which the phoneme representations are assumed to be degraded (Vellutino et al., 2004; Ziegler \& Goswami, 2005). It is believed that these assumedly degraded phoneme representations hamper the acquisition of stable letter speech sound associations, which is considered a prerequisite for the development of fluent reading and writing skills (Ehri, 2005; Frith, 1985). This is explicitly proposed as a cascade effect in a recent review of studies on the development of dyslexia (McCandliss \& Noble, 2003). However, the causal role of a phonological deficit in dyslexic reading problems has been questioned (Castles \& Coltheart, 2004; Morais, Bertelson, Cary, \& Alegria, 1986; Morais, Cary, Alegria, \& Bertelson, 1979; Perfetti, Beck, Bell, \& Hughes, 1987; Wagner, 1987; Wimmer, Landerl, Linortner, \& Hummer, 1991). It has even been advanced that phonological problems are rather a consequence than a cause of deficient reading in dyslexia (Wimmer et al., 1991). In a longitudinal study, auditory and phonological perception of pre-school children with low and high risk for dyslexia was tested (Boets, Wouters, Wieringen, \& Ghesquiere, 2007). Despite clear group differences, analyses on the individual level showed that phonological processing was neither a sufficient nor a necessary condition to cause reading problems. In line with this, a recent pre-school training study reported that a core group of children genetically at risk for dyslexia had specific letter - speech sound processing problems, while their phonological awareness was at the level of children not at risk of dyslexia (Willems, Poelmans, Richardson, \& Blomert, submitted).

Remarkably, since letter - speech sound processing plays an important role in the widely accepted phonological deficit theory as the cause of reading problems in dyslexia, only two studies have directly investigated letter - speech sound processing in dyslexia directly (Blau et al., 2009; Blomert \& Vaessen, 2009). Moreover, to our knowledge, until now there is no neural record of letter - speech sound processing in children with dyslexia. The study presented in chapter 4 therefore aims at adding to our understanding of dyslexia by presenting electrophysiological correlates of letter - speech sound processing in children with dyslexia.

\section{MMN}

When certain brain areas are activated, e.g. during speech perception, a small current crosses the neural membrane of neurons within this area. This evokes an electric potential in the conductive media in- and outside the neurons. When synchronous activation of neuronal populations within an area is sufficiently high, the common electric potential resulting from the currents generated by the activated 
neurons can be measured by placing electrodes at the scalp and executing an electroencephalogram (EEG). An event related potential (ERP) is obtained by averaging all those electric potentials time-locked to specific single events repeated a number of times during an experiment, e.g. speech sound presentation. Each ERP is a twodimensional wave-form which can be described by the amplitude of each positive or negative peak at a certain latency in this wave-form. The advantages of measuring ERPs is that it can be performed non-invasively and has a high temporal resolution. Unfortunately, the spatial resolution is low. Although source localization can indicate possible areas involved, the "inverse problem" makes it hard to determine the exact source of the electrical signal (Kutas \& Dale, 1997; Kutas \& Schmitt, 2003).

A number of ERP components have been described based on their latency and topographical distribution over the scalp, e.g. early components like P1, N1 and P2 reflecting obligatory, exogenous processing, more endogenous components like $\mathrm{N} 2 \mathrm{~b}$ and P3 reflecting attention related processing or later components like N400, P600 and LAN reflecting content related processing (Kutas \& Dale, 1997; Kutas \& Schmitt, 2003). A well established and frequently used ERP component is the mismatch negativity (MMN). The MMN is an automatic deviance detection mechanism, mostly described in the auditory domain (Näätänen, 1995). The MMN is known to be evoked between 100 and $200 \mathrm{~ms}$ when, in a sequence of auditory stimuli, a rarely presented sound (the deviant) deviates in one or more aspects from the sound that is frequently presented (the standard). The MMN is considered to reflect the neurophysiological correlate of a comparison process between an incoming auditory stimulus and the memory trace formed by the repetitive aspect of the standard stimulus (Näätänen, 2000; Picton et al., 2000; Schröger, 1998).

The MMN has repeatedly been shown to be sensitive to language-specific speech sound representations in adults and in children (Bonte, Mitterer, Zellagui, Poelmans, \& Blomert, 2005; Bonte, Poelmans, \& Blomert, 2007; Mitterer \& Blomert, 2003; Näätänen, 2001; Winkler et al., 1999). The child MMN is furthermore suggested to be a stable component resembling the adult MMN (Csépe, 2003), and is particularly interesting and useful for research with children, because its evocation does not require sustained attention or a task. Moreover, the MMN has been used before to investigate phonological and auditory processing deficits in children and adults with dyslexia (Bishop, 2007; Bonte et al., 2007; Csépe, 2003; Kujala \& Naatanen, 2001). A clear advantage of employing the $M M N$ is that a sound discrimination function can be determined in a passive way and more directly than with behavioral measures.

The MMN has been used before to investigate neural correlates of audiovisual integration (Besle, Fort, \& Giard, 2005; Colin et al., 2002; de Gelder, Bocker, Tuomainen, Hensen, \& Vroomen, 1999; Möttönen, Krause, Tiippana, \& Sams, 2002; Sams et al., 1991; Stekelenburg, Vroomen, \& de Gelder, 2004). Until now, however, these studies investigated audiovisual processing in which a deviant visual stimulus evoked an 
MMN by illusorily changing the percept of the standard sound (Besle et al., 2005). Consequently, the MMN reflects what is also measurable at a behavioral level. In the present thesis, a cross-modal MMN paradigm appears furthermore a valid tool to investigate neural correlates of more subtle cross-modal integration in the human brain.

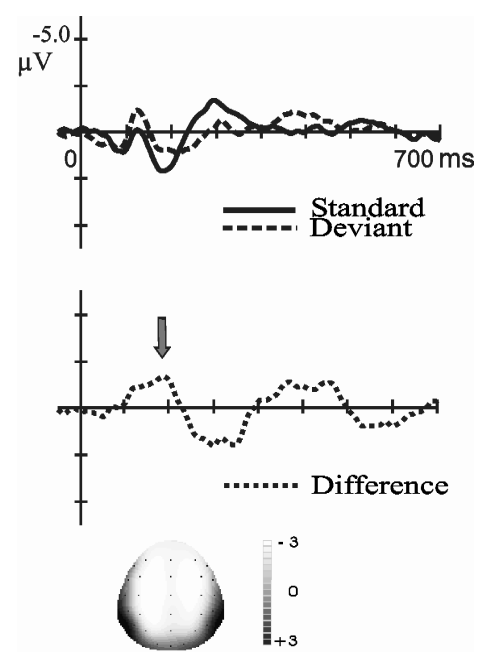

Figure 1. Example of the MMN as measured at electrode Fz: differences between grand average waves to standard and deviant sounds (top) are reflected in a difference wave (middle) not equal to zero. The topographical distribution of the entire scalp at the MMN peak (indicated with the arrow) reveals the typical reversal of polarity at the mastoids (bottom).

Recently a number of studies have found converging evidence for the existence of a visual counterpart of the aMMN (Berti \& Schröger, 2003; Czigler, 2007; Czigler, Balazs, \& Pato, 2004; Heslenfeld, 2003; Kimura, Katayama, Ohira, \& Schröger, 2009; Maekawa et al., 2005; Pazo-Alvarez et al., 2003; Tales, Newton, Troscianko, \& Butler, 1999). The visual MMN (vMMN) is described as a negativity measured at the occipital electrodes between 150 and 350 msec after the onset of an infrequent (deviant) visual stimulus in a sequence of frequently presented (standard) visual stimuli (Pazo-Alvarez et al., 2003). The vMMN is suggested to have similar properties as the aMMN. It can be evoked pre-attentively and it reflects the use of a memory representation of regularities in visual stimulation (Czigler, 2007). The neural sources are suggested to reside in the extrastriate areas of the brain (Czigler et al., 2004). The properties of the $\mathrm{VMMN}$ make it an appropriate tool to look for automatic influences of speech sounds on letter processing and investigate the time course of these influences. 
In sum, in the present thesis one visual and four auditory MMN studies are presented in which the time course and automaticity of letter - speech sound processing are investigated.

Chapter two: In the study presented in this chapter the automaticity and time course of letter - speech sound processing is investigated in fluently reading adults. The auditory MMN is employed since it is known to be evoked automatically between 100 to $250 \mathrm{~ms}$ after sound onset. A speech sound oddball was presented either in isolation or simultaneously with letters. If the experienced reader automatically integrates letters and speech sounds, we expect this to be reflected in a cross-modal influence of the letter on the MMN to speech sounds. Further, the time window of integration was investigated by presenting letters either simultaneously or $\mathbf{1 0 0}$ or $\mathbf{2 0 0}$ ms before speech sound onset. That is because in an fMRI-study investigating the neural network for letter - speech sound processing, indications were found for a narrow time window of integration.

Chapter three: In a subsequent study the development of letter - speech sound processing was investigated. Two age groups participated; eight year old children with at least one year of reading instruction and eleven year old children with at least four years of reading instruction. In a transparent language like Dutch, letter speech sound knowledge is acquired within months of reading instruction, so we expected to find letter - speech sound integration effects in both age groups. However, since reaction times to letter - speech sound identification and discrimination still develop after years of reading instruction, we also expected this development to be reflected in the cross-modal effect of letters on the MMN to speech sounds. Again we investigated the time window of integration; in the audiovisual condition, letters were presented either simultaneously or $200 \mathrm{~ms}$ before speech sound onset.

Chapter four: To directly investigate letter - speech sound processing in children with dyslexia, once more the same cross-modal MMN paradigm was employed. We investigated dyslexic children of eleven years old, in this way obtaining an age match with the oldest group in the developmental study (presented in chapter three) and a reading level match with the youngest group in that study. We focused on the early MMN time window, but also on a later time window that revealed to be interesting in the developmental study. Correlations with behavioral measures were calculated. Eventual correlations would underpin the letter - specificity of the reported effects. Comparing these data with age and reading level matched groups allowed to disentangle maturational from reading level dependent effects.

Chapter five: In this chapter, we used the visual counterpart of the auditory MMN, the visual $\mathrm{MMN}$, to investigate the influence of speech sounds on letter processing. If there is a symmetric involvement of both the auditory and visual low level sensory areas, we expect to find the reverse from what was found in the cross-modal auditory MMN studies, namely a cross-modal influence of speech sounds on letter proc- 
essing. Results are discussed in perspective of earlier findings on audiovisual speech processing and general audiovisual processing.

Chapter six: In order to validate the cross-modal MMN paradigm, used in three of the four previous studies, in this chapter we replicated and extended the previous results with different stimuli and a visual control stimulus. Three conditions were created; a speech sound only condition, the same speech sounds presented with letters and the same speech sounds presented with non-letters. The non-letter control stimulus is a scrambled version of the letter used in the audiovisual letter condition. If the previously measured cross-modal effect on the MMN was genuine, we expect again an influence of the letter on speech sound processing. If this crossmodal effect is letter - specific, we expect no or less influence of the non-letter on the MMN to speech sounds. Additionally, correlations between the letter - specific MMN effect and behavioral reading measures were calculated. Eventual correlations would add to the relevance of the effect and help with interpretating it.

\section{References}

Aarnoutse, C., van Leeuwe, J., \& Verhoeven, L. (2000). Ontwikkeling van beginnende geletterdheid (development of beginning literacy). Pedagogische Studiën, 77, 307 - 325.

Berti, S., \& Schröger, E. (2003). Distraction effects in vision: Behavioral and event-related potential effects. Neuroreport, 15, 665-669.

Besle, J., Fort, A., \& Giard, M. (2005). Is the auditory memory sensitive to visual information. Experimental Brain Research, 166, 337-344.

Bishop, D. V. M. (2007). Using mismatch negativity to study central auditory processing in developmental language and literacy impairments: Where are we, and where should we be going? Psychological bulletin, 133, 651-672.

Blau, V., Atteveldt, N., Ekkebus, M., Goebel, R., \& Blomert, L. (2009). Reduced neural integration of letters and speech sounds links phonological and reading deficits in adult dyslexia. Current Biology, 19, 503 508.

Blau, V., van Atteveldt, N., Formisano, E., Goebel, R., \& Blomert, L. (2008). Task-irrelevant visual letters interact with the processing of speech sounds in heteromodal and unimodal cortex. European journal of neuroscience, 28(3), 500-509.

Blomert, L. (2005). Dyslexie in Nederland - theorie, praktijk en beleid (Dyslexia in The Netherlands - theory, practice and policy). Amsterdam: Nieuwezijds Publishers.

Blomert, L., \& Vaessen, A. (2009). 3DM Differential diagnostics for dyslexia: Cognitive analysis of reading and spelling. Amsterdam: Boom Test Publishers.

Boets, B., Wouters, J., Wieringen, A., \& Ghesquiere, P. (2007). Auditory processing, speech perception and phonological ability in pre-school children at high-risk for dyslexia: A longitudinal study of the auditory temporal processing theory. Neuropsychologia, 45, 1608-1620.

Bonte, M., Mitterer, H., Zellagui, N., Poelmans, H., \& Blomert, L. (2005). Auditory cortical tuning to statistical regularities in phonology. Clinical Neurophysiology, 116(12), 2765-2774.

Bonte, M., Poelmans, H., \& Blomert, L. (2007). Deviant neurophysiological responses to phonological regularities in speech in dyslexic children. Neuropsychologia, 45, 1427-1437.

Calvert, G. A., Brammer, M. J., \& Iversen, S. D. (1998). Crossmodal identification. Trends in Cognitive Sciences, 2, 247-253.

Castles, A., \& Coltheart, M. (2004). Is there a causal link from phonological awareness to succes in learning to read? Cognition, 91, 77 - 111. 
Colin, C., Radeau, M., Soquet, A., Demolin, D., Colin, F., \& Deltenre, P. (2002). Mismatch negativity evoked by the McGurk-MacDonald effect: a phonetic representation within short-term memory. Clin Neurophysiol, 113(4), 495-506.

Coltheart, M., Rastle, K., Perry, C., Langdon, R., \& Ziegler, J. (2001). DRC: A dual route cascaded model of visual word recognition and reading aloud. Psychological review, 108, 204 - 256.

Csépe, V. (2003). Dyslexia: different Brain, Different Behavior. New York: Kluwer Academic/ Plenum Publishers.

Czigler, I. (2007). Visual mismatch negativity; Violation of nonattended environmental regularities. Journal of Psychophysiology, 21, 224-230.

Czigler, I., Balazs, L., \& Pato, L. (2004). Visual change detection: event-related potentials are dependent on stimulus location in humans. Neuroscience letters, 364, 149-153.

de Gelder, B., Bocker, K. B., Tuomainen, J., Hensen, M., \& Vroomen, J. (1999). The combined perception of emotion from voice and face: early interaction revealed by human electric brain responses. Neuroscience Letters, 260(2), 133-136.

Dijkstra, T., Schreuder, R., \& Frauenfelder, U. H. (1989). Grapheme Context Effects on Phonemic Processing. Language and Speech, 32, 89-108.

Ehri, L. C. (2005). Development of sight word reading: phases and findings. In M. J. Snowling \& C. Hulme (Eds.), The science of reading: A handbook (pp. 135 - 145). Oxford: Blackwell Publishing.

Frith, U. (1985). Beneath the surface of developmental dyslexia. In K. E. Patterson, J. C. Marshall \& M. Coltheart (Eds.), Surface dyslexia. London: Routledge \& Kegan-Paul.

Hardy, M. H., Smythe, P. C., Stennet, R. G., \& Wilson, H. R. (1972). Developmental patterns in elemental reading skills: phoneme-grapheme and grapheme-phoneme correspondences. Journal of Educational Psychology, 63, 433-436.

Hashimoto, R., \& Sakai, K. L. (2004). Learning letters in adulthood: direct visualization of cortical plasticity for forming a new link between orthography and phonology. Neuron, 42(2), 311-322.

Herdman, A. T., Fujioka, T., Chau, W., Ross, B., Pantev, C., \& Picton, T. W. (2006). Cortical oscillations related to processing congruent and incongruent grapheme-phoneme pairs. Neuroscience Letters, 399, 61 - 66.

Heslenfeld, D. J. (2003). Visual mismatch negativity. In J. Polich (Ed.), Detection of change: Event related potential and fMRI findings. Boston: Kluver Academic Press.

Kimura, M., Katayama, J., Ohira, H., \& Schröger, E. (2009). visual mismatch negativity: New evidence from the equiprobable paradigm. Psychophysiology, 46, 402-409.

Kujala, T., \& Naatanen, R. (2001). The mismatch negativity in evaluating central auditory dysfunction in dyslexia. Neurosci Biobehav Rev, 25(6), 535-543.

Kutas, M., \& Dale, A. (1997). Electrical and magnetic readings of mental functions. In M. D. Rugg (Ed.), Cognitive Neuroscience (pp. 197 - 243). Hove East Sussex: Psychology Press.

Kutas, M., \& Schmitt, B. (2003). Language in Microvolts. In M. T. Banich \& M. A. Mach (Eds.), Mind, Brain \& Language: multidisciplinary perspectives (pp. 171-203). San Diego: Erlbaum Ass. Inc.

Lyon, G. R., Shaywitz, S. E., \& Shaywitz, B. A. (2003). Towards a definition of dyslexia. Annals of Dyslexia, 53, 1-14.

Maekawa, T., Goto, Y., Kinukawa, N., Taniwaki, T., Kanba, S., \& Tobimatsu, S. (2005). Functional characterization of mismatch negativity to a visual stimulus. Clinical neurophysiology, 116, 2392-2402.

Marsh, G., Friedman, M., Welch, V., \& Desberg, P. (1981). A cognitive-developmental theory of reading acquisition. In G. E. MacKinnon \& T. G. Waller (Eds.), Reading research: advances in theory and practice. New York: Academic Press.

McCandliss, B. D., \& Noble, K. G. (2003). The development of reading impairment: a cognitive neuroscience model. Ment Retard Dev Disabil Res Rev, 9(3), 196-204.

Mitterer, H., \& Blomert, L. (2003). Coping with phonological assimilation in speech perception: evidence for early compensation. Percept Psychophys, 65, 956-969.

Morais, J., Bertelson, P., Cary, L., \& Alegria, J. (1986). Literacy training and speech segmentation. cognition, $24,45-64$. 
Morais, J., Cary, L., Alegria, J., \& Bertelson, P. (1979). Does awareness of speech as a saquence of phones arise spontaneously? Cognition, 49, 957 - 958.

Möttönen, R., Krause, C. M., Tiippana, K., \& Sams, M. (2002). Processing of changes in visual speech in the human auditory cortex. Brain Res Cogn Brain Res, 13(3), 417-425.

Näätänen, R. (1995). The Mismatch Negativity: A powerful tool for cognitive neuroscience. Ear and Hearing, 16, 6-18.

Näätänen, R. (2000). Mismatch negativity (MMN): perspectives for application. Int J Psychophysiol., 37, 3-10.

Näätänen, R. (2001). The perception of speech sounds by the human brain as reflected by the mismatch negativity (MMN) and its magnetic equivalent (MMNm). Psychophysiology, 38, 1-21.

Pazo-Alvarez, P., Cadaveira, F., \& Amenedo, E. (2003). MMN in the visual modality: a review. Biol Psychol, 63(3), 199-236.

Perfetti, C. A., Beck, I., Bell, L., \& Hughes, C. (1987). Phonemic knowledge and learning to read are reciprocal: A longitudinal study of first grade children. Merrill-Palmer Quarterly, 33, 283-319.

Picton, T. W., Alain, C., Otten, L., Ritter, W., \& Achim, A. (2000). Mismatch negativity: different water in the same river. Audiol Neurootol, 5, 111-139.

Raij, T., Uutela, K., \& Hari, R. (2000). Audiovisual integration of letters in the human brain. Neuron, 28, 617625.

Sams, M., Aulanko, R., Hamalainen, M., Hari, R., Lounasmaa, O. V., Lu, S. T., et al. (1991). Seeing speech: visual information from lip movements modifies activity in the human auditory cortex. Neurosci Lett, 127(1), 141-145.

Schneider, W., \& Chein, J. M. (2003). Controlled \& automatic processing: behavior, theory and biological mechanisms. Cognitive Science, 27, 525-559.

Schröger, E. (1998). Measurement and interpretation of the mismatch negativity. Behavior Research Methods Instruments \& Computers, 30, 131-145.

Siegel, L. S., \& Faux, D. (1989). Aquisition of certain grapheme-phoneme correspondences in normally achieving and diabled readers. Reading and Writing, an interdisciplinary journal, 1, 37-52.

Snowling, M. J. (1980). The development of grapheme-phoneme correspondence in normal and dyslexic readers. Journal of Experimental Child Psychology, 29, 294-305.

Stekelenburg, J. J., Vroomen, J., \& de Gelder, B. (2004). Illusory sound shifts induced by the ventriloquist illusion evoke the mismatch negativity. Neuroscience Letters, 357, 163-166.

Tales, A., Newton, P., Troscianko, T., \& Butler, S. (1999). Mismatch negativity in the visual modality. Neuroreport, 10(16), 3363-3367.

Van Atteveldt, N., Formisano, E., Blomert, L., \& Goebel, R. (2007). The effect of temporal asynchrony on the multisensory integration of letters and speech sounds. Cerebral Cortex, 13, 962-974.

Van Atteveldt, N., Formisano, E., Goebel, R., \& Blomert, L. (2004). Integration of letters and speech sounds in the human brain. Neuron, 43, 271-282.

Van Atteveldt, N., Formisano, E., Goebel, R., \& Blomert, L. (2007). Top-down task effects overrule automatic multisensory responses to letter-sound pairs in auditory association cortex. Neurolmage, 36, 13451360.

Vellutino, F. R., Fletcher, J. M., Snowling, M. J., \& Scanlon, D. M. (2004). Specific reading disability (dyslexia): what have we learned in the past four decades? J Child Psychol Psychiatry, 45(1), 2-40.

Wagner, R. K., Torgesen, J.K. (1987). The nature of phonological processing and its causal role in the acquisition of reading skills. Psychological Bulletin, 101, 192-212.

Willems, G., Poelmans, H., Richardson, U., \& Blomert, L. (submitted). What preschool intervention in children at familial risk of dyslexia reveals about their core deficits and the way to treat them.

Wimmer, H., Landerl, K., Linortner, R., \& Hummer, P. (1991). The relationship of phonemic arareness to reading acquisition: More consequence than precondition but still important. Cognition, 40, 219-249.

Winkler, I., Kujala, A., Tiitinen, H., Sivonen, P., Alku, P., Lehtokoski, A., et al. (1999). Brain responses reveal the learning of foreign language phonemes. psychophysiology, 36, 638-642.

Ziegler, J. C., \& Goswami, U. (2005). Reading acquisition, developmental dyslexia, and skilled reading across languages: a psycholinguistic grain size theory. Psychol Bull, 131(1), 3-29. 
Chapter 2

\section{Cross-modal enhancement of the MMN to speech sounds indicates early and automatic integration of letters and speech sounds} the MMN to speech sounds indicates early and automatic integration of letters and speech sounds. Neuroscience Letters, 430, $23-28$. 


\begin{abstract}
Recently brain imaging evidence indicated that letter - speech sound integration, necessary for establishing fluent reading, takes place in auditory association areas and that the integration is influenced by stimulus onset asynchrony (SOA) between the letter and the speech-sound. In the present study we used a specific ERP measure known for its automatic character, the mismatch negativity (MMN), to investigate the time course and automaticity of letter - speech sound integration. We studied the effect of visual letters and SOA on the MMN elicited by a deviant speech-sound. We found a clear enhancement of the MMN by simultaneously presenting a letter, but without changing the auditory stimulation. This enhancement diminishes linearly with increasing SOA. These results suggest that letters and speech sounds are processed as compound stimuli early and automatically in the auditory association cortex of fluent readers and that this processing is strongly dependent on timing.
\end{abstract}




\section{Introduction}

Learning to read is a multistage process that presupposes the establishment of associations between letters and speech sounds. Most models describing the process of learning to read emphasize the generation of letter to speech-sound connections as a crucial first step in the learning process (Ehri, 2005). At least $90 \%$ of school children learn the relation between letter - speech sound pairs within a few months without exceptional effort (Blomert, 2005). At the same time we also know that failure of this step in reading acquisition is considered a main cause for reading problems like in developmental dyslexia (Vellutino, Fletcher, Snowling, \& Scanlon, 2004). Snowling (1980) showed that dyslexic readers revealed a weaker developmental performance increase on a letter - speech sound matching task in comparison with their normally reading peers (Snowling, 1980), indicating that fluent reading probably requires full automatization of letter - speech sound associations.

Despite the importance for acquiring fluent reading skills, the behavioral and neural characteristics of letter - speech sound associations have hardly been investigated. Dijkstra and colleagues (1989) tested the influence of congruent and incongruent letter primes on the performance in a speech-sound monitoring task. The results revealed faster identification of congruent in comparison with incongruent letter primes, thus indicating automatic influences of letters on speech-sound processing (Dijkstra, Schreuder, \& Frauenfelder, 1989). Raij, Uutela \& Hari (2000) conducted a magneto-encephalography (MEG) study to investigate the neural time course of letter - speech sound integration (Raij, Uutela, \& Hari, 2000). Simultaneously presented congruent letters and speech sounds suppressed brain activations in the superior temporal sulcus (STS) around $345 \mathrm{~ms}$ after stimulus onset in comparison with the sum of brain activations in response to the unimodal stimuli, indicating letter - speech sound integration in this area. Recently, letter - speech sound processing was investigated with $\mathrm{fMRI}$ to obtain a more precise localization of the neural mechanisms involved in their integration (Van Atteveldt, Formisano, Blomert, \& Goebel, 2007; Van Atteveldt, Formisano, Goebel, \& Blomert, 2004). The results of a first fMRI study showed that not only STS and superior temporal gyrus (STG) responding to heteromodal stimuli, but also auditory association cortex (planum temporale, PT) responding only to auditory stimuli, were crucially involved in the neural binding of letters and speech sounds (Van Atteveldt et al., 2004). Besides enhanced activation in response to congruent and incongruent presentations in STS and STG, enhancement for congruent and suppression for incongruent presentations were found in PT. This result was replicated in a subsequent study in which the stimulus onset asynchrony (SOA) between letters and speech sounds was varied (Van Atteveldt et al., 2007). The results revealed significant interactions between SOA and congruency in the PT, indicating temporal synchrony to be critical for the integration of letters and speech sounds in this auditory area. The modulation of auditory proc- 
essing by letters in the PT occurred within a very narrow time window with already declining influence at $150 \mathrm{~ms}$ SOA.

In sum, letter - speech sound integration seems to be performed by a network of the PT and the STS/STG, and seems dependent on a narrow time-window of integration. Given the passive nature of the tasks used in the fMRI studies (passive viewing/listening), letter - speech sound integration seems to occur automatically.

In the present study we further investigate the automaticity and SOA-dependency of letter - speech sound integration. We used the mismatch negativity (MMN), because it is evoked early and automatically (Näätänen, Paavilainen, Tiitinen, Jiang, \& Alho, 1993). This allows investigating the automatic character of letter - speech sound associations with high temporal accuracy. If in a sequence of auditory stimuli a rarely presented sound (the deviant) deviates in one or more aspects from the sound that is frequently presented (the standard), an MMN is evoked between 100 and $200 \mathrm{~ms}$ after stimulus onset. The MMN is considered to reflect the neurophysiological correlate of a comparison process between an incoming auditory stimulus and the memory trace formed by the repetitive aspect of the standard stimulus (Schröger, 1998). It is generally assumed that a major MMN source is located in the primary auditory cortex, which might be related to the sensory memory mechanism, but additional frontal generators have been described (Ahlo, 1995; Giard, 1990). The MMN has repeatedly been shown to be sensitive to phonemic processing (Näätänen, 2001).

In the present report, we presented an oddball paradigm with speech sounds in two different contexts. We compared the MMN evoked by deviant speech sounds (auditory experiment) with the MMN evoked by the same deviant speech sounds combined with visually presented letters (audiovisual experiment). We conducted 4 different experiments, one auditory and three audiovisual experiments. In all four experiments subjects passively listened to the speech sounds /a/ (standard, 90\%) and /o/ (deviant, 10\%). In the auditory experiment subjects listened to these speech sounds while watching a silent movie. In the three audiovisual experiments subjects listened to the same speech sounds, while watching single letters. The three audiovisual experiments differ only with regard to the stimulus onset asynchrony (SOA) between the presentation of the letter and speech-sound. The letter always appeared first and the speech-sound respectively 0, 100 or 200 ms later. We will from now on refer to the three audiovisual experiments as AV_0, AV_100 and AV_200.

We first hypothesized that the deviant speech-sound would elicit a typical MMN (auditory experiment). Second, we expected that the letter - speech sound pair is automatically processed as a compound stimulus in the auditory cortex. As a consequence, we hypothesized that the presence of a visual letter may influence the MMN response to the deviant speech-sound (audiovisual experiment). Third, if there is an influence, we predicted that it may be sensitive to a temporal asyn- 
chrony between the letter and speech-sound as indicated by the strong SOA effect found in van Atteveldt et al. (Van Atteveldt et al., 2007). We chose $100 \mathrm{~ms}$ and 200 ms SOA since these SOAs include the significantly effective SOA of $150 \mathrm{~ms}$ used in Van Atteveldt et al. (Van Atteveldt et al., 2007). The fact that the major MMN source is located in the early auditory cortex (Ahlo, 1995; Giard, 1990), including the planum temporale where the reported effect of SOA was most pronounced (Van Atteveldt et al., 2007), underpinned our prediction.

\section{Methods}

\section{Participants}

Fourteen subjects participated in the auditory experiment (11 female, range 18 to 33 , mean age 21.92 years). Twelve subjects participated in AV_0 ( 9 female, range 20 to 33, mean age 24.69 years). Thirteen subjects participated in AV_100 (9 female, range 19 to 29, mean age 22.38 years). Seventeen subjects participated in AV_200 (13 female, range 18 to 33, mean age 23.05 years). Three subjects participated in all four experiments. Another 11 subjects participated both in the auditory experiment and in AV_200. Subjects gave informed written consent and were paid for their participation. All subjects were undergraduate university students without any history of hearing or reading problems and with normal or corrected to normal vision. One subject was left handed and participated in the auditory experiment and in AV_200.

\section{Stimuli}

Stimuli were natural speech sounds, /a/ (384 ms) and /o/ (348 ms), and the visually presented single letter "a". speech sounds were digitally recorded (sampling rate $44.1 \mathrm{kHz}, 16$ bit quantization) from a female speaker. Recordings were band-pass filtered (180-10.000 Hz) and resample at $22.05 \mathrm{kHz}$ and matched for loudness with Praat software (Boersma \& Weenink, 2002). The sounds were presented binaurally through loudspeakers at about $65 \mathrm{~dB}$ SPL. The letter was presented in white on a black background in the centre of a computer screen for $500 \mathrm{~ms}$, printed in lower case font "Arial" at letter size 40. Between the presentations of the stimuli a white fixation cross was presented in the centre of the screen. To ensure that subjects were at all times focusing the screen, we interspersed the experimental trials with a non-related color picture. Subjects had to press a button when they saw the picture. Since the focusing task was very easy and not related to the stimuli of interest we suggest that this did not influence the MMN, a very basic deviance detection mechanism of the brain (Schröger, 1998), problematically (see discussion). Each experiment consisted of 4 experimental blocks with 534 trials. Trial length was always $1250 \mathrm{~ms}$. When subjects heard the speech-sound deviant /o/, we always pre- 
sented the standard letter "a". Although we also manipulated the visual stimuli in some trials, these conditions are not relevant for the present study and are reported elsewhere (Froyen, in preparation).

\section{Recording and analysis}

EEG data were recorded with NeuroScan 4.2 from 0.01 to $50 \mathrm{~Hz}$ with a sampling rate of $250 \mathrm{~Hz}$ in a sound-attenuating and electrically shielded room from 30 electrode positions (Extended International 10-20 system) relative to a nose reference. Eye-movements and blinks were measured with bipolar VEOG/HEOG channels. All electrode impedance levels (EEG and EOG) were kept below $5 \mathrm{k} \Omega$. EEG data were epoched from -50 to $850 \mathrm{~ms}$ relative to stimulus onset, baseline corrected $(50 \mathrm{~ms}$ pre-stimulus interval), and $1-30 \mathrm{~Hz}$ band pass filtered with NeuroScan 4.2. Epochs containing data exceeding a maximum voltage criterion of $75 \mu \mathrm{V}$ were rejected. Standards immediately following deviants were not included in the analyses, as were the trials with target pictures. The raw EEG data were corrected for vertical eye-movements (i.e. blink artifacts). In the auditory experiment we included 146 (73\%) of the standard trials and $151(75 \%)$ of the deviant trials. In AV_0 we retained respectively 184 (92\%) and 185 (93\%) of the trials, in AV_100 182 (91\%) and 186 (93\%) and in AV_200 180 (90\%) and 182 (91\%). Epochs were averaged separately for each condition and each participant from the onset of the visual stimulus.

Difference waves were calculated by subtracting the ERPs to the standard condition from ERPs to the deviant condition. In the audiovisual experiments, the visual stimulus was always the same in the standard and the deviant conditions (the letter "a"). Therefore, the same visual brain wave will be evoked in both conditions. Since the auditory stimulus was always presented simultaneously with or later than the letter, the visual evoked potentials elicited by the letters can be subtracted out by calculating deviant minus standard difference waves.

To investigate the MMN, statistical analyses were performed on 9 electrodes covering frontal, central, centroparietal and temporal areas (Fz, Cz, FC3, FC4, T3, T4, CP3, CP4 and $\mathrm{Pz}$ ), followed by a detailed analysis on the frontocentral electrodes ( $F z, C z, F C 3$, FC4), where the MMN effect was most prominent. Timing of ERP responses in the MMN window was measured by determining individual peak latencies between 50 and $250 \mathrm{~ms}$ after stimulus onset. This time-window was set after visual inspection of the data and includes the time-window (100 to $200 \mathrm{~ms}$ ) typically reported in MMNstudies (Schröger, 1998). Amplitude measures included both individual peak amplitude and mean amplitude taken from the $50 \mathrm{~ms}$ window around the individual peak latency (separately for each subject, condition and electrode). When performing further analysis of the difference waves on the frontocentral electrodes we excluded two subjects with Z-scores on all four electrodes higher than 1.96 or lower than -1.96 . We only report results reaching significance at $p<0.05$ after Bonferroni correction. 


\section{Results}

Auditory experiment. We first analyzed the auditory experiment to probe a typical MMN evoked by a deviant speech-sound in a standard speech-sound context. The left part of Figure 1 shows the grand average ERPs (A) and difference waveforms (deviant-standard trials) (B) on Fz for the speech-sound deviant in the auditory experiment. The speech-sound deviant elicited an MMN with average peak latency of $168 \mathrm{~ms}$ (averaged over the four frontocentral electrodes, range: 94-238 ms) after the onset of the auditory stimulus deviation (Figure 2), with a topographical distribution that is typically reported (left part Figure $1 \mathrm{C}$ ). A polarity reversal of the MMN at electrode sites below the sylvian fissure (left part Figure $1 \mathrm{C}$ ) suggests main neural generators in the auditory cortex (Schröger, 1998).

\section{Auditory experiment}

(A)

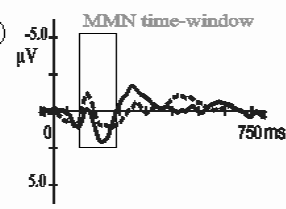

(B)

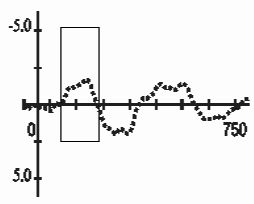

(C)

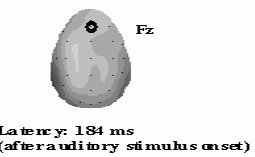

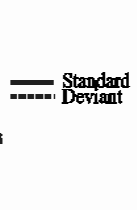
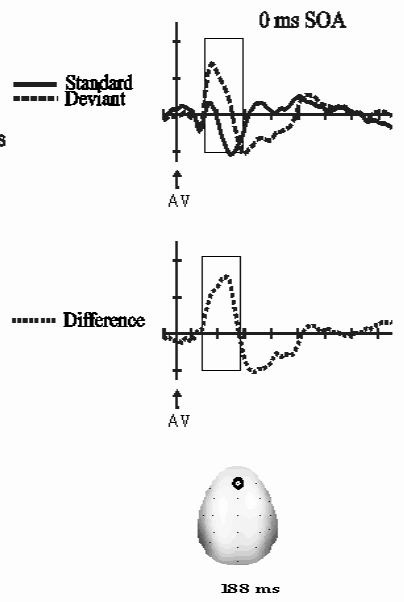

Audiovisual experiments
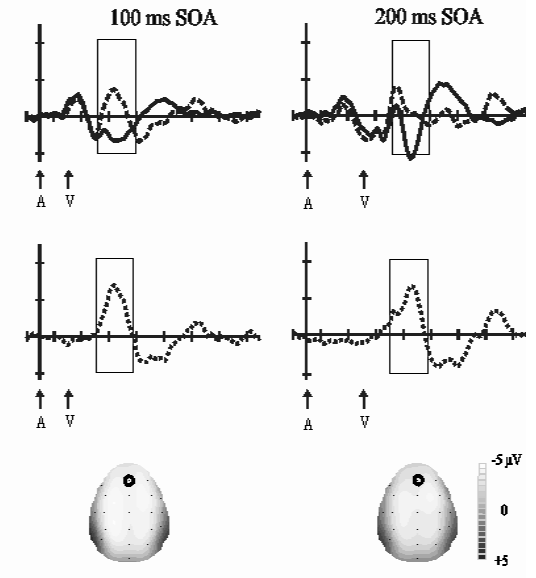

$182 \mathrm{~ms}$

Figure 1. Grand average ERPs at Fz for the standard and deviant stimuli (A), difference waves (B) and MMN maps $(C)$ for the auditory experiment and the three audiovisual experiments. Arrows indicate the onset of visual (V) and auditory (A) stimuli and the grey rectangle indicates the MMN-time window.

Amplitude characteristics of ERP activity in the MMN time-window were first analyzed using a 2 (stimulus type; standard versus deviant) by 9 (electrode sites) repeated-measures ANOVA. We found an interaction between stimulus type and electrode site for mean, $\mathrm{F}(8,104)=3.0, p=0.004$, and peak values, $\mathrm{F}(8,104)=2.0, p$ $=0.05$. Further analysis was performed on four frontocentral electrodes using a 2 (stimulus type; standard versus deviant) by 4 (electrode sites) repeated-measures ANOVA. There was no interaction with electrode site for mean, $F(3,39)=0.6, p=$ 0.587 , and peak values, $F(3,39)=.6, p=0.58$. We found a marginal effect of stimulus type for mean values, $F(1,13)=4.4, p=0.055$ and an effect of stimulus type for peak values, $F(1,13)=5.4, p=0.037$. The deviant grand average waves are system- 
atically more negative in comparison with standard grand average waves. Further analyses of the difference waves were performed with one-sample t-tests on each of the frontocentral electrodes, after exclusion of one subject (Z-scores of 2.20 for Fz, 2.15 for Cz, 2.0 for FC3 and 2.1 for FC4). Mean and peak amplitude values were significantly different from zero; for mean amplitudes $\mathrm{Fz}, \mathrm{t}(12)=-11.7 ; p<.001, \mathrm{Cz}$, $\mathrm{t}(12)=-10.5 ; p<.001, \mathrm{FC}, \mathrm{t}(12)=-8.6 ; p<.001$ and $\mathrm{FC} 4, \mathrm{t}(12)=-12.3 ; p<.001$ and for peak amplitudes Fz, $\mathrm{t}(12)=-11.7 ; p<.001, \mathrm{Cz}, \mathrm{t}(12)=-10.5 ; p<.001, \mathrm{FC}, \mathrm{t}(12)=-$ 8.6; $p<.001$ and $\mathrm{FC} 4, \mathrm{t}(12)=-12.3 ; p<.001$.

Audiovisual experiment. In all 4 experiments ( 1 auditory and 3 audiovisual experiments with different SOAs) the deviant speech-sound stimulus elicited a large negativity around 180 ms after auditory stimulus onset (grey rectangle in Figure 1B), with a typical topographical distribution of the MMN (Figure 1C). Average peak latencies (averaged over the four frontocentral electrodes) were $169 \mathrm{~ms}$ (range: 118-206 ms) for AV_0, 177 ms (range: 126-214 ms) for AV_100 and 172 ms (range: 104-208 ms) for AV_200 after the onset of the auditory stimulus deviation (Figure 2).

Amplitude and latency characteristics of ERP activity in the MMN time-window were first analyzed using a mixed ANOVA with two within-subject factors; stimulus type (2) and electrode sites (9). The between group variable was experiment with 4 levels for the 4 different experiments. We found an interaction between stimulus type and electrode site for mean, $F(8,416)=7.4, p<0.001$, and peak values, $F(8,416)=22.7$, $p<0.001$. We performed further analysis on four frontocentral electrodes using a 2 (stimulus type; standard versus deviant) by 4 (electrode sites) repeated-measures ANOVA with experiment as between subject variable. There was no interaction of stimulus type with electrode site for mean, $\mathrm{F}(9,156)=0.2, p=0.370$, and peak values, $F(9,156)=1.2, p=0.306$. As expected, deviant stimuli elicited a significantly stronger negative ERP response than standard stimuli (main effect of stimulus type for mean amplitude, $\mathrm{F}(1,52)=103.7 ; p<0.001$, and for peak amplitude, $\mathrm{F}(1,52)=$ 103.7; $p<0.001)$. Crucially, this mismatch effect showed a significant interaction with experiment (stimulus type-by-experiment interaction for mean amplitude, $\mathrm{F}(3,52)=6.1 ; p=0.001$, and for peak amplitude, $\mathrm{F}(3,52)=6.1 ; p=0.01)$.

For further statistical analysis of the difference waves on the frontocentral electrodes we excluded one subject of the auditory experiment (Z-scores of $2.20 \mathrm{for} F \mathrm{~F}$, 2.15 for $\mathrm{Cz}, 2.0$ for FC3 and 2.1 for FC4) and one subject of AV_100 (Z-scores of -2.23 for $\mathrm{Fz},-2.6$ for $\mathrm{Cz},-2.8$ for $\mathrm{FC} 3$ and -2.3 for FC4). Thirteen subjects were preserved in the auditory experiment ( 10 female, range 18 to 33 , mean age 21.84 years). Twelve subjects were preserved in AV_100 (9 female, range 19 to 29, mean age 22.61 years). A one-way ANOVA on mean values revealed a significant effect of experiment in all four electrodes, $F(3)=4.9, p=0.004$ for $F z, F(3)=4.1, p=0.011$ for $C z$, $\mathrm{F}(3)=5.9, p=0.001$ for $\mathrm{FC} 3$ and $\mathrm{F}(3)=3.7, p=0.018$, for $\mathrm{FC} 4$, the same result was 
found for peak area values, $F(3)=4.4, p=0.008$ for $F z, F(3)=4.6, p=0.007$ for $\mathrm{Cz}$, $F(3)=5.6, p=0.002$ for $F C 3$ and $F(3)=3.3, p=0.027$, for FC4. The MMN was stronger in the audiovisual experiments in comparison with the $\mathrm{MMN}$ in the auditory experiment (Figure 2). Post hoc t-tests (Bonferroni corrected) on mean area values showed that the effect of experiment was due to a difference between the auditory experiment and AV_0, $p<0.001$ for Fz, $p=0.003$ for $\mathrm{Cz}, p<0.001$ for FC3, $p$ $=0.006$ for FC4, the same is true for peak values, $p=0.002$ for Fz, $p=0.001$ for $\mathrm{Cz}, p$ $<0.001$ for FC3, $p=0.004$ for FC4. For electrodes Fz, $p=0.045$, and CP3, $p=0.030$, we also found a difference between AV_0 and AV_200. For all frontocentral electrodes the $M M N$ in $A V_{-} 0$ was significantly larger than the $M M N$ in the auditory experiment. For $\mathrm{Fz}$ and $\mathrm{CP} 3$ the $\mathrm{MMN}$ in $\mathrm{AV}_{-} 0$ was significantly larger than the MMN in AV_200. To test whether the decrease in MMN amplitude was linear over the three audiovisual experiments we performed a polynomial contrast with the four frontocentral electrodes as a within subject factor. This analysis revealed a significant linear contrast for both mean, $p=0.026$, and peak values, $p=0.045$. Finally, a repeated measures ANOVA with the four frontal electrodes as a within subject factor and experiment as a between subject factor was performed to test for an effect of latency. There was no interaction between latency and electrodes, $\mathrm{F}(9,147)=1.2, p=0.307$, and no main effect of latency, $\mathrm{F}(3,49)=.36, p=0.781$.

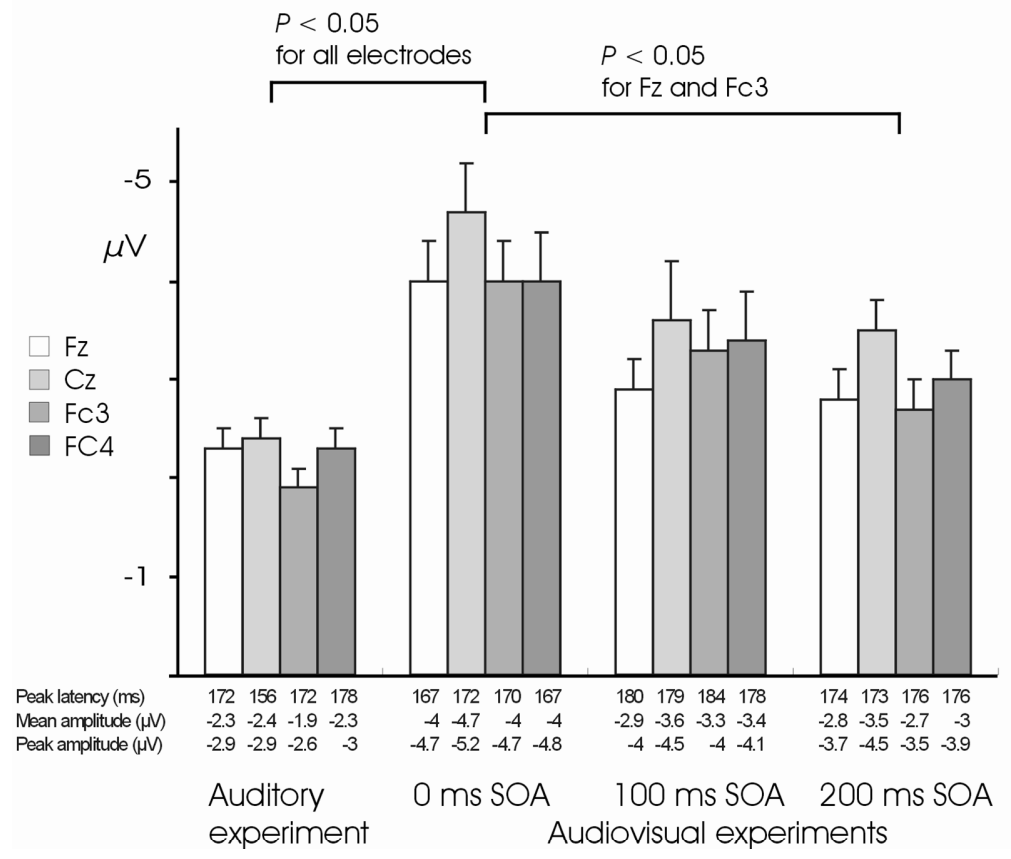

Figure 2. Mean amplitude values over $50 \mathrm{~ms}$ around the individually assessed MMN peak with standard error for the four frontocentral electrodes Fz, Cz, FC3 and FC4, including latency, peak and mean amplitude values. 


\section{Discussion}

To summarize, we used the MMN to investigate the automaticity and timing characteristics of the influence of letters on the processing of speech sounds. We observed the influence of letters on the difference wave evoked in an auditory oddball paradigm with speech sounds and investigated the influence of stimulus onset asynchrony (SOA). In a purely auditory experiment, a typical auditory MMN was shown. We compared the auditory MMN with the negativity in the difference waves evoked in the audiovisual experiments (audiovisual MMN) and found a general enhancement of the amplitude, decreasing linearly with increasing SOA.

We hypothesized early and automatic integration of letters and speech sounds. When simultaneously presented with the speech-sound, we found a clear influence of the letter on the MMN evoked by the deviant speech-sound; an enhancement of the MMN amplitude. This enhancement might be explained by the learned letter speech sound association, causing a double deviation in the audiovisual experiments: the deviant speech-sound differs from the standard speech-sound and the letter on the screen. The MMN is typically evoked between 100 and 200 ms after stimulus onset (Näätänen, 2001; Schröger, 1998). This indicates that the influence of the letter on the ERP-difference wave reflecting speech-sound processing is established early in the processing of the speech-sound. Since the MMN is known to be evoked automatically (Näätänen et al., 1993), and there were no task requirements related to the letter or speech-sound stimuli, our findings furthermore suggest that the integration of letters and speech sounds occurs automatically. It remains though to be investigated to what extent a neutral (nonlinguistic) visual stimulus might influence the MMN evoked by a deviant speech-sound.

As mentioned before, we observed an effect of SOA within the audiovisual experiments. There was a linear trend of decreasing enhancement of the audiovisual MMN amplitude with increasing SOA. In Fz and FC3, the MMN in AV_0 differs significantly from the MMN in AV_200. Since the MMN at AV_100 did not differ significantly from the MMN in the auditory experiment, we suggest that even $100 \mathrm{~ms}$ SOA is enough to prevent complete processing of letters and speech sounds as compounds. A similar effect of timing in the auditory association cortex was found in a previous fMRI study by our group (2007) (Van Atteveldt et al., 2007). This region is assumed to be the location of the main neural generators of the MMN (Ahlo, 1995; Giard, 1990), with additional frontal generators. The consistent findings from different methods (ERP and $\mathrm{fMRI}$ ) strengthen the hypothesis that temporal proximity is a critical factor for cross-modal integration of letters and speech sounds (Van Atteveldt et al., 2007). Apparently, once arbitrarily related cross-modal associations become over-learned, integration is subject to very basic rules such as temporal proximity (Meredith, Nemitz, \& Stein, 1987). The narrow time window of integration might be a consequence of the rigidity and inflexibility of the letter - speech sound integration process. Consistently, this integration process seems to fulfill the 
main criteria for automatic processing in general as formulated by Schneider \& Chein (Schneider \& Chein, 2003); letter - speech sound integration is also described as fast, robust to distractors and requiring low effort .

The MMN is suggested to be influenced by attention in the context of an active task (Oades, 1995). In the present experiment, the active task in the audiovisual experiments only served as focusing control and was completely unrelated to the processing of the letter and speech-sound stimuli. Moreover, the linear decrease of the audiovisual MMN amplitude with increasing SOA cannot be explained by the presence or absence of the focusing task, since the task was the same in all audiovisual experiments. Furthermore, at all four frontocentral electrodes the MMN at AV_200 (focusing task) was no longer significantly higher in comparison with the MMN in the auditory experiment (no focusing task). This indicates that the focusing task is not exclusively responsible for the enhancement of the MMN.

It is still possible that the presence of letters involuntary drew attention to the auditory stimuli. However, in absence of active task demands with regard to the letter and speech-sound stimuli, such an involuntary switch of attention might still be interpreted as automatic. Our suggestion is in line with our previous fMRI results of interactions between letters and speech sounds in a completely passive design (Van Atteveldt et al., 2007; Van Atteveldt et al., 2004).

Considering possible contamination of the auditory evoked potentials by visual evoked potentials we checked the brain waves in the audiovisual experiments before and after the onset of the auditory stimulus (Figure 1). Prior to auditory stimulus onset, the presented letter was identical in the standard and deviant conditions and accordingly elicited comparable visual evoked responses, resulting in the absence of a difference potential prior to auditory stimulus onset in AV_100 and AV_200. After auditory stimulus onset, the morphology of the evoked responses is comparable across the three AV experiments: including a P1-N1-P2 and a sustained negativity (both in the standard and the deviant conditions). This similarity in evoked responses indicates the absence of a general modulation by overlapping visual and auditory evoked potentials at frontocentral electrodes.

\section{Conclusion}

In conclusion, we found a clear effect of letters on the processing of speech sounds as reflected in the MMN. This result indicates that letter - speech sound pairs are processed early and automatically as compound stimuli in normally reading adults. The present study provides a method to non-invasively investigate automatic letter - speech sound integration, which might be applied in research on children of all ages. Schneider and Chein's criteria imply that automaticity is an effect of extended training. In future research, it will therefore be interesting to investigate the emergence of automaticity of letter - speech sound associations in beginning readers. 


\section{Acknowledgments}

This work was supported by grant 608/002/2005 of the Dutch Board of Health Care Insurance (College voor Zorgverzekeringen) awarded to L.B. We thank Hanne Poelmans and Vera Blau for assistance in data acquisition.

\section{References}

Ahlo, K. (1995). Cerebral Generators of mismatch negativity (MMN) and its magnetic counterpart (MMNm) elicited by sound changes. Ear \& Hearing, 16(1), 38-51.

Blomert, L. (2005). Dyslexie in Nederland - theorie, praktijk en beleid (Dyslexia in The Netherlands - theory, practice and policy). Amsterdam: Nieuwezijds Publishers.

Boersma, P., \& Weenink, D. (2002). Praat 4.0: a system for doing phonetics with the computer. Amsterdam: Universiteit van Amsterdam.

Dijkstra, T., Schreuder, R., \& Frauenfelder, U. H. (1989). Grapheme Context Effects on Phonemic Processing. Language and Speech, 32, 89-108.

Ehri, L. C. (2005). Development of sight word reading: phases and findings. In M. J. Snowling \& C. Hulme (Eds.), The science of reading: A handbook (pp. 135 - 145). Oxford: Blackwell Publishing.

Froyen, D., Van Atteveldt, N, Bonte, $\mathrm{M}$ and Blomert, L. (in preparation).

Giard, M. H., Perrin, F, Pernier J, Bouchet P. (1990). Brain generators implicated in the processing of auditory stimulus deviance: a topographic event-related potential study. Psychophysiology, 27, 627640.

Meredith, M. A., Nemitz, J. W., \& Stein, B. E. (1987). Determinants of multisensory integration in superior colliculus neurons. I. Temporal factors. Journal of Neuroscience, 7(10), 3215-3229.

Näätänen, R. (2001). The perception of speech sounds by the human brain as reflected by the mismatch negativity (MMN) and its magnetic equivalent (MMNm). Psychophysiology, 38, 1-21.

Näätänen, R., Paavilainen, P., Tiitinen, H., Jiang, D., \& Alho, K. (1993). Attention and mismatch negativity. Psychophysiology, 30, 436-350.

Oades, R. D., Dittmann-Balcar, A. (1995). Mismatch negativity (MMN) is altered by directing attention. Neuroreport, 6, 1187-1190.

Raij, T., Uutela, K., \& Hari, R. (2000). Audiovisual integration of letters in the human brain. Neuron, 28, 617-625.

Schneider, W., \& Chein, J. M. (2003). Controlled \& automatic processing: behavior, theory and biological mechanisms. Cognitive Science, 27, 525-559.

Schröger, E. (1998). Measurement and interpretation of the mismatch negativity. Behavior Research Methods Instruments \& Computers, 30, 131-145.

Snowling, M. J. (1980). The development of grapheme-phoneme correspondence in normal and dyslexic readers. Journal of Experimental Child Psychology, 29, 294-305.

Van Atteveldt, N., Formisano, E., Blomert, L., \& Goebel, R. (2007). The effect of temporal asynchrony on the multisensory integration of letters and speech sounds. Cerebral Cortex, 13, 962-974.

Van Atteveldt, N., Formisano, E., Goebel, R., \& Blomert, L. (2004). Integration of letters and speech sounds in the human brain. Neuron, 43, 271-282.

Vellutino, F. R., Fletcher, J. M., Snowling, M. J., \& Scanlon, D. M. (2004). Specific reading disability (dyslexia): what have we learned in the past four decades? J Child Psychol Psychiatry, 45(1), 2-40. 
Chapter 3

\section{The Long road to automation: Neurocognitive development of letter - speech sound processing}




\begin{abstract}
In transparent, alphabetic languages, the expected standard for complete acquisition of letter - speech sound associations is within one year of reading instruction. The neural mechanisms underlying the acquisition of letter - speech sound associations have, however, hardly been investigated. The present paper describes an event-related potential (ERP) study with beginner and advanced readers in which the influence of letters on speech sound processing is investigated by comparing the mismatch negativity ( $\mathrm{MMN}$ ) to speech sounds presented in isolation with the MMN to speech sounds accompanied by letters. Furthermore, stimulus onset asynchrony (SOA) between letter and speech sound presentation was manipulated in order to investigate the development of the temporal window of integration for letter speech sound processing.

Beginner readers, despite one year of reading instruction, showed no early letter speech sound integration, i.e. no influence of the letter on the evocation of the MMN to the speech sound. Only later in the difference wave, at $650 \mathrm{~ms}$, was an influence of the letter on speech sound processing revealed. Advanced readers, with four years of reading instruction, showed early and automatic letter - speech sound processing as revealed by an enhancement of the MMN-amplitude, however at a different temporal window of integration in comparison with experienced adult readers.

The present results indicate a transition from mere association in beginner readers to more automatic, but still not 'adult-like', integration in advanced readers. In contrast to general assumptions, the present study provides evidence for an extended development of letter - speech sound integration.
\end{abstract}




\section{Introduction}

The acquisition of letter - speech sound associations is considered the basic requirement for learning to read (Ehri, 2005; Frith, 1985). Additionally, it is suggested that deficient letter - speech sound association forms the basis for the difficulties in learning to read in developmental dyslexia (Fox, 1994; Frith, 1985; Snowling, 1980; Vellutino, Fletcher, Snowling, \& Scanlon, 2004). A better understanding of the development of this association process will provide insights in the cognitive requirements for learning to read and will contribute to the research of reading disabilities like developmental dyslexia.

Since written language is a relatively recent cultural invention, an evolutionarily adapted brain mechanism for processing script most likely does not exist. Moreover, in contrast with the natural association between auditory and visual information in, for example audiovisual speech (Calvert, Brammer, \& Iversen, 1998), the association between letters and speech sounds is basically arbitrary in nature. It is therefore remarkable that literacy skills are so easily acquired in most languages. In a relatively transparent language like Dutch, in which the letter - speech sound associations are mostly unambiguous, the expected educational standard for complete mastery of letter - speech sound associations is within one year of reading instruction (Wentink \& Verhoeven, 2003). Even in English, an opaque language regarding the associations between orthography and phonology, letter - speech sound associations seem to be established within a few years of reading instruction (Hardy, Smythe, Stennet, \& Wilson, 1972; Siegel \& Faux, 1989; Snowling, 1980). In a study in which children had to decide whether single letters matched with single speech sounds, most letter - speech sound associations were judged correctly by at least $80 \%$ of all children within one year of reading instruction (Hardy et al., 1972).

The seemingly easy acquisition of letter - speech sound associations needs however closer examination, since recent data indicate that knowing the associations between letters and speech sounds might not be the same as the instrumental use of this association in learning to read. In a study on a large nationwide sample of Dutch primary school children from six to twelve years old, not only accuracy but also reaction time data on letter - speech sound association were collected (Blomert \& Vaessen, 2009). Surprisingly, in the light of the expected mastery of letter - speech sound associations within one year of reading instruction, reaction times of letter speech sound discrimination decisions steadily decreased during the whole range of Dutch primary school reading instruction. Since faster processing is one of the main criteria for the automation of a process (Schneider \& Chein, 2003), the extended decrease of processing time for letter - speech sound discrimination might indicate an extended development towards automatic letter - speech sound association. While early acquisition of letter - speech sound associations is generally assumed in transparent languages, exactly when and how these associations develop in the human brain remains an open question, and is the topic of the present paper. 
One of the first indications about the nature of fully developed letter - speech sound processing came from an early behavioral study showing differential influences of congruent and incongruent letter primes on reaction times in a speech sound identification task (Dijkstra, Schreuder, \& Frauenfelder, 1989). More recently, in a brain imaging study using magneto encephalography (MEG), integration of letters and speech sounds was reported in the superior temporal sulcus (STS) (Raij, Uutela, \& Hari, 2000). Moreover, two fMRI-studies revealed that not only heteromodal regions in STS and superior temporal gyrus (STG) but also the unimodal auditory association cortex (planum temporale, PT) is involved in the letter - speech sound integration process (Van Atteveldt, Formisano, Blomert, \& Goebel, 2007; Van Atteveldt, Formisano, Goebel, \& Blomert, 2004). The involvement of the auditory cortex for letter - speech sound integration was recently confirmed in an MEGstudy (Herdman et al., 2006). Given the passive nature of the tasks used in both fMRI studies (passive viewing/listening), letter - speech sound integration was suggested to occur automatically. Van Atteveldt et al. (2007) furthermore manipulated the stimulus onset asynchrony (SOA) between the presentation of the letter and the speech sound. Manipulating the SOA revealed a narrow temporal window for integration of letters with speech sounds in PT. Integration, as reflected by response enhancement for congruent and suppression for incongruent letter - speech sound pairs (relative to the maximal unimodal response), was maximal at simultaneous presentation of letters with speech sounds and had already declined at $150 \mathrm{~ms}$ SOA (Van Atteveldt et al., 2007). To summarize, current evidence indicates that fully developed letter - speech sound processing in adults is achieved through automatic integration performed by a network located in the PT and the STS/STG, where the multisensory effect in PT only occurs within a narrow temporal window under the condition that letters and speech sounds are presented simultaneously.

These recent insights into the brain mechanisms involved in fully automated letter speech sound processing open new perspectives for the investigation of its development. Knowing the associations between letters and speech sounds is apparently only the starting point of the development towards automatic letter - speech sound integration. In the present study, we therefore further investigated when and how letter - speech sound processing develops by means of event related potentials (ERP), known for its high temporal resolution. In a recent ERP-study, the mismatch negativity (MMN) was successfully used to investigate the automaticity and timing properties of letter - speech sound integration in experienced adult readers (Froyen, van Atteveldt, Bonte, \& Blomert, 2008). The MMN is known to be evoked automatically between 100 and 200 ms when, in a sequence of auditory stimuli, a rarely presented sound (the deviant) deviates in one or more aspects from the sound that is frequently presented (the standard) (Näätänen, Paavilainen, Tiitinen, Jiang, \& Alho, 1993). The MMN is considered to reflect the neurophysiological correlate of a comparison process between an incoming auditory stimulus and the 
memory trace formed by the repetitive aspect of the standard stimulus (Näätänen, 2000; Picton, Alain, Otten, Ritter, \& Achim, 2000; Schröger, 1998). The MMN has repeatedly been shown to be sensitive to language-specific speech sound representations in adults and in children (Bonte, Poelmans, \& Blomert, 2007; Mitterer \& Blomert, 2003; Näätänen, 2001; Winkler et al., 1999). The child MMN is furthermore suggested to be a stable component resembling the adult MMN (Csépe, 2003). The MMN is particularly interesting and useful for research with children, because its evocation does not require sustained attention.

In an MMN-study with experienced adult readers, the MMN evoked by the deviant speech sound violating the standard speech sound (Figure $1 \mathrm{~A}$ ) was compared with the MMN evoked by the deviant speech sound violating both the standard speech sound and the corresponding letter (Figure 1B) (Froyen et al., 2008). The results revealed a clear enhancement of the $M M N$ amplitude evoked in the audiovisual experiment in comparison with the MMN amplitude in the auditory experiment. Considering the properties of the MMN, this enhancement strongly points to automatic integration of letters and speech sounds. Froyen et al. (2008) also manipulated the stimulus onset asynchrony (SOA) between the presentation of the letter and the speech sound. Only when the letter was presented simultaneously with the speech sound did the letter have an influence on the processing of the speech sound, while at $100 \mathrm{~ms}$ SOA the influence of the letter diminished significantly. The consistent SOA-effect found with different methods, ERP (Froyen et al., 2008) and fMRI (Van Atteveldt et al., 2007), strongly points to temporal proximity as a critical factor for automatic letter - speech sound integration.

\section{Present study}

Since the present study aims at determining when and how letter - speech sound integration develops, we used the same design and ERP-measurement with beginner and advanced readers as used in the study with adult readers (Froyen et al., 2008). In order to investigate the development of the temporal window for integration, we included two different SOAs; letters and speech sounds were presented simultaneously or with an SOA of $200 \mathrm{~ms}$. The temporal integration window describes the time-span within which two physically separated events are perceived as appearing together (Van Wassenhove, Grant, \& Poeppel, 2007; Yabe et al., 2004; Yabe et al., 1998). To our knowledge there are no studies investigating the development of the temporal integration window in letter - speech sound perception. There is however some evidence that children between 8-12 years of age perceive an auditory and a visual stimulus as occurring simultaneously in a wider temporal window than adults do (Laasonen, Service, \& Virsu, 2002; Laasonen, Tomma-Halme, Lahti-Nuuttila, Service, \& Virsu, 2000). To provide insight into the dynamics of the development of letter - speech sound associations we investigated the temporal 
window of integration for letter - speech sound processing in two different age groups in whom letter - speech sound processing might still be developing. We included two SOAs in the present study, the SOA for which the adults revealed maximal integration and the SOA for which we found no integration at all (Froyen et al., 2008).

First, we hypothesized that a typical MMN would be elicited to the speech sounds in both beginner (one year of reading instruction) and advanced readers (four years of reading instruction). Second, as the educational standard for fully mastering letter speech sound associations is expected to be reached within one year of reading instruction (Wentink \& Verhoeven, 2003), we predicted an influence of the letter on the MMN evoked to a speech sound in both age groups. Third, the decreasing reaction time for letter - speech sound associations over the whole primary school range (Blomert \& Vaessen, 2009) may be reflected in a MMN-latency difference between beginner and advanced readers. This would be in line with previously reported decreasing MMN response latencies over development (Bonte et al., 2007; Maurer, Bucher, Brem, \& Brandeis, 2003; Shafer, Morr, Kreuzer, \& Kurtzberg, 2000). Finally, 'adult-like' letter - speech sound integration is achieved with a narrow temporal window of integration only if stimuli are presented simultaneously (Froyen et al., 2008; Van Atteveldt et al., 2007). The results from Laasonen et al. $(2000,2002)$ suggest that we might expect a wider temporal window for letter - speech sound processing for advanced readers (11 years old) than for experienced adult readers (Froyen et al., 2008; Van Atteveldt et al., 2007). This might be reflected in appropriate integration at $200 \mathrm{~ms}$ SOA and $0 \mathrm{~ms}$ SOA for beginner and advanced readers, whereas adults only integrate at 0 ms SOA (Froyen et al., 2008; Van Atteveldt et al., 2007).

\section{Methods}

\section{Participants}

Sixty-four normally reading subjects participated in the present study. To investigate developmental effects of letter - speech sound associations, two age groups were created. Thirty-eight subjects were recruited from grade 2 , having received one year of reading instruction. Thirteen subjects participated in the auditory experiment (three female, range 7;01 to 8;10, mean age 7;08 years), fourteen subjects participated in the audiovisual experiment with 0 ms SOA ( 8 female, range 7;06 to 9, mean age 8;02 years) and seventeen subjects participated in the audiovisual experiment with 200 ms SOA (7 female, range 6;11 to 9 years, mean age 7;06 years). Six subjects participated in two experiments, the auditory experiment and the audiovisual experiment with $0 \mathrm{~ms}$ SOA. Data of two subjects in the audiovisual experiment with $200 \mathrm{~ms}$ SOA were discarded due to excessive movement artifacts. Twenty-six sub- 
jects were recruited from grade 5 , having received four years of reading instruction. Twelve subjects participated in the auditory experiment ( 3 females, range 10;06 to $12 ; 09$ years, mean age 11;04 years), thirteen subjects participated in the audiovisual experiment with $0 \mathrm{~ms}$ SOA (9 female, range 10 to 12;09 years, mean age 11;02 years), fourteen subjects participated in the audiovisual experiments with $200 \mathrm{~ms}$ SOA ( 7 females, range 10;02 to 11;08 years, mean age 11;01 years). Seven subjects participated in both the auditory experiment and the audiovisual experiment with 0 ms SOA, four subjects participated in the auditory experiment and the audiovisual experiment with $200 \mathrm{~ms}$ SOA. Data of three subjects (one in the audiovisual experiment with 0 ms SOA and two in the audiovisual experiment with $200 \mathrm{~ms}$ SOA) were discarded due to excessive movement artifacts.

Table 1.

Descriptive data (mean and standard deviation) for the two age groups in each experiment.

\begin{tabular}{lllll}
\hline & A & AV 0 ms & AV 200 ms & Differences \\
\hline $\begin{array}{llll}\text { Beginner readers: } \\
\text { Word reading }^{\mathrm{a}}\end{array}$ & $12.8(2.0)$ & $13.9(1.5)$ & $12.5(2.4)$ & $p=0.228$ \\
Non-word reading $^{\mathrm{a}}$ & $12.6(1.7)$ & $14.5(2.2)$ & $12.5(2.0)$ & $p=0.066$ \\
Ravens SS $^{\mathrm{b}}$ & $7.2(1.5)$ & $6.9(1.8)$ & $6.5(1.7)$ & $p=0.421$ \\
Advanced readers: & $13.1(1.8)$ & $13.1(2.4)$ & $12(1.4)$ & \\
Word reading $^{\mathrm{a}}$ & $12.9(2.1)$ & $12.8(3.4)$ & $12.4(1.9)$ & $p=0.307$ \\
Non-word reading $^{\mathrm{a}}$ & $7.5(1.3)$ & $7.6(1.1)$ & $7.1(0.6)$ & $p=0.883$ \\
Ravens SS $^{\mathrm{b}}$ & & & & \\
\hline
\end{tabular}

IQ scores and performance on language tests (mean); $\mathrm{A}=$ auditory experiment, $\mathrm{AV}=$ audiovisual experiment ${ }^{\text {a }}$ Age- appropriate norms (standardized scale; 0-20, mean $=10, S D=2$ )

${ }^{\mathrm{b}}$ Age- appropriate norms (standardized scale; $0-10$, mean $=5, \mathrm{SD}=2$ )

All children were given a present for participation. Informed consent was obtained from the children and the parents. Approval for the study was granted by the Ethical Committee of the Faculty of Psychology at the University of Maastricht. The children were recruited from local schools in The Netherlands, and were judged by their school teachers to be average or above average in school achievement. All subjects were native Dutch speakers. Subject results of behavioral tests are summarized in Table 1. IQ assessment was established with the Raven Colored Progressive Matrices (Raven, 1998). All children performed standardized language tests, the Eénminuut-leestest (Brus \& Voeten, 1973) and a non-word reading test (Van den Bos, Lutje Spelberg, Scheepsma, \& De Vries, 1999). Results of the behavioral tests showed no differences in $\mathrm{IQ}$, word reading or phonological decoding (non-word reading) between the groups of the same age participating in the different experiments (Table 1). 
Stimuli

Stimuli were speech sounds, /a/ (384 ms) and /o/ (348 ms), and the visually presented single letter "a". We used the same stimuli as used in the adult study (Froyen et al., 2008) and in an MMN-study on gender differences in speech and non speech processing (Kasai et al., 2002). To ensure a typical MMN reflecting speech sound processing, we used natural vowels (Näätänen, 2001). Furthermore, we chose the vowels "o" and "a" as these are completely transparent in their letter - speech sound correspondence in Dutch, whereas other vowels are slightly inconsistent ("u", " $e$ " and " $y$ ") or visually divergent ("i").

Speech sounds were digitally recorded (sampling rate $44.1 \mathrm{kHz}, 16$ bit quantization) from a Dutch female speaker. Recordings were band-pass filtered (180-10.000 $\mathrm{Hz}$ ), resampled at $22.05 \mathrm{kHz}$ and matched for loudness with Praat software (Boersma \& Weenink, 2002). The sounds were presented binaurally through loudspeakers at about $65 \mathrm{~dB}$ SPL. The letter was presented in white on a black background in the centre of a computer screen for $500 \mathrm{~ms}$, printed in lower case font "Arial" at font size 40, approximately $1.5 \mathrm{~cm}$ high and $1 \mathrm{~cm}$ wide. The distance from the screen to the subject was kept at $70 \mathrm{~cm}$. Consequently stimuli had a visual angle of approximately 1.2 by 0.8 degrees. During fixation periods, a white fixation cross was presented in the centre of the screen. The visually presented letter was always an "a", irrespective of whether the standard speech sound /a/ or the deviant speech sound /o/ was presented.

\section{Design and procedure}

In total, we conducted three different experiments, one auditory and two audiovisual experiments. In all three experiments subjects listened to the speech sounds /a/ (standard, 90\%) and /o/ (deviant, 10\%). In the auditory experiment subjects listened to these speech sounds while watching a silent movie (Figure 1A). In the two audiovisual experiments subjects listened to the same speech sounds, while watching single letters (Figure 1B). Children were instructed not to move their eyes and sit quietly. To ensure subjects consistent perception of the letters, in the audiovisual experiments, periodically a full color picture of a present appeared in between trials. Children were instructed to press a button when this picture was presented. The two audiovisual experiments differ only with regard to the stimulus onset asynchrony (SOA) between the presentation of the letter and the speech sound. The letter appeared simultaneously (AV_0) with the speech sound or 200 ms earlier (AV_200). Each experiment consisted of 4 experimental blocks with 534 trials each. Trial length was always $1250 \mathrm{~ms}$. 
A) Auditory experiment

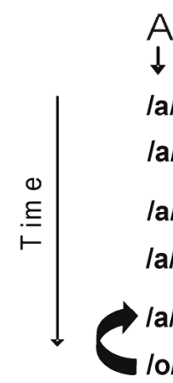

B) Audiovisual experiments

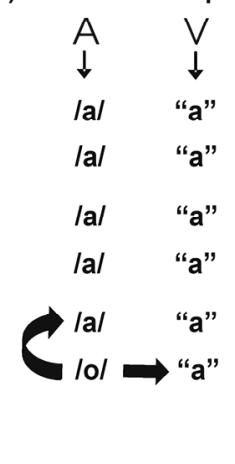

Figure 1. Designs of the auditory experiment (A) and the audiovisual experiment (B). ' $A$ ' represents auditory stimulus presentation, ' $\mathrm{V}$ ' represents visual stimulus presentation. The arrow indicates the violation of the standard speech sound in the auditory experiment $(A)$ and the violation of both the standard speech sound and the letter in the audiovisual experiment (AV).

\section{Recording and analysis}

EEG data were recorded in a sound-attenuating and electrically shielded room $(0.01$ - $50 \mathrm{~Hz}$, sampling rate $250 \mathrm{~Hz}$ ) from 30 electrode positions (Extended International 10-20 system, (Nuwer et al., 1998)) relative to a nose reference. Eye-movements and blinks were measured with bipolar VEOG/HEOG channels. All electrode impedance levels (EEG and EOG) were kept below $5 \mathrm{k} \Omega$. EEG data were epoched from -50 to $850 \mathrm{~ms}$ relative to stimulus onset, baseline corrected $(50 \mathrm{~ms}$ pre-stimulus interval), and $1-30 \mathrm{~Hz}$ band pass filtered (NeuroScan 4.2). Epochs containing data exceeding a maximum voltage criterion of $75 \mu \mathrm{V}$ were rejected. Standards immediately following deviants were not included in the analyses. The raw EEG data were corrected for vertical eye-movements (i.e. blink artifacts; (Semtlitsch, Anderer, Schuster, \& Presslich, 1986)). Subjects with over 30\% rejected epochs, i.e. 60 epochs on a total of 200 per condition, were excluded from analysis. Considering the beginner readers: in the auditory experiment, the mean number of accepted epochs in the grand averages for standards and deviants were 162 (14) and 161 (15); in AV_0 respectively 172 (20) and 174 (19); in AV_200 respectively 163 (21) and 163 (17). Considering the advanced readers: in the auditory experiment, the mean number of accepted epochs in the grand averages for standards and deviants were 173 (17) and 173 (17); in AV_0 respectively 174 (19) and 177 (18); in AV_200 respectively $178(20)$ and $179(18)$. The resulting cleaned data sets did not show any noticeable confounds due to motion or eye-movement artifacts and accordingly no noticeable age group differences. Epochs were averaged separately for each condi- 
tion and each participant. Difference waves are calculated by subtracting the ERPs to the standard condition from ERPs to the deviant condition.

Statistical analyses were performed on 11 electrodes covering the frontocentral ( $\mathrm{Fz}$, $\mathrm{Cz}, \mathrm{FC} 3, \mathrm{FC} 4)$, temporal (T3 and T4), parietal (CP3 and CP4) and occipital (Oz, O1 and O2) regions of the brain to investigate the auditory MMN. From the difference waveforms we derived two dependent variables for each electrode: (I) the MMN latency measured at the maximum amplitude of the $\mathrm{MMN}$ in the latency range from 50-250 ms post stimulus onset and (II) the MMN amplitude measured as the mean amplitude across $50 \mathrm{~ms}$ centered around the individual peak latency (separately for each subject, condition and electrode). In the present study we used the mean area amplitude rather than the peak amplitude as it is considered a more reliable measure. Consequently, in the present study, the term amplitude always refers to the mean area amplitude. The analysis on 11 electrodes was followed by a detailed analysis on the frontocentral electrodes (Fz, Cz, FC3, FC4), where the MMN effect was most prominent. All reported $p$-values from the repeated measures ANOVA were Greenhouse-Geisser corrected.

\section{Results}

\section{Beginner readers}

Auditory experiment: We first analyzed the auditory experiment to probe a typical $M M N$ evoked by a deviant speech sound in a standard speech sound context. Figure 2A shows grand average ERPs for standard and deviant stimuli and difference waveforms (deviant - standard stimuli) obtained in the auditory experiment as measured at the frontocentral electrode sites (Fz, Cz, FC3, FC4). The speech sound deviant elicited an MMN with average peak latency of $155 \mathrm{~ms}$ (averaged over the four frontocentral electrodes, $53 \mathrm{~ms}$ SD) after the onset of the auditory stimulus, with a topographical distribution (Figure $2 \mathrm{~A}$ ) that is typically reported for the MMN (Picton et al., 2000; Schröger, 1998). 


\section{Beginner readers}

\section{A. Auditory experiment}

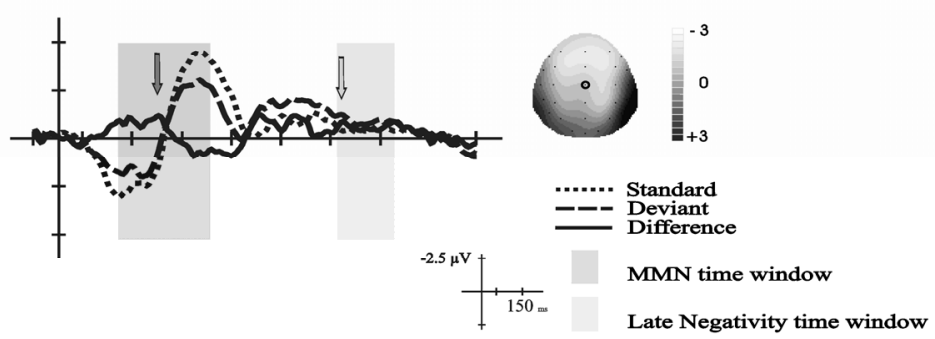

B. Audiovisual experiment 0 ms SOA
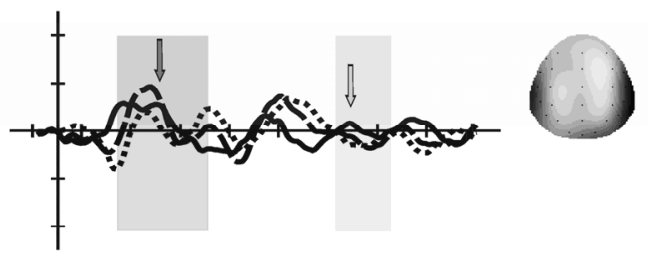

\section{Audiovisual experiment $200 \mathrm{~ms}$ SOA}

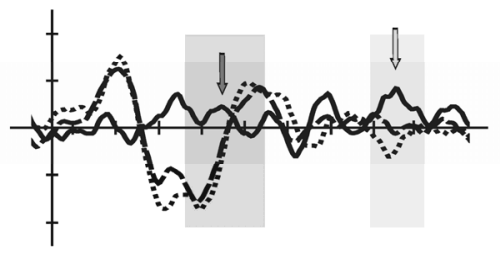

Figure 2. Grand average ERPs for the standard (dotted lines) and deviant stimuli (dashed lines), difference waves (solid lines) as measured at $\mathrm{Cz}$, and MMN maps for the auditory experiment $(\mathrm{A})$ and the two audiovisual experiments $0 \mathrm{~ms}$ SOA (B) and $200 \mathrm{~ms}$ SOA (C) with beginner readers.

Amplitude properties of ERP activity in the MMN time-window were first analyzed using a 2 (stimulus type; standard versus deviant) by 11 (electrode sites) repeatedmeasures ANOVA. We found a marginally significant interaction with electrode site, $F(10,120)=2.95 ; p=0.056$ (Figure 3 ). Since we expected an interaction of stimulus type with electrode, we further analyzed only the four frontocentral electrodes, where the MMN is expected to be most pronounced. The same analysis on only the four frontocentral electrodes revealed no significant interaction between stimulus type and electrode, $F(3,36)=7.58 ; p=0.46$, and a main effect of stimulus type for mean amplitude values in the $50 \mathrm{~ms}$ window around the individual peaks, $F(1,12)=$ 7.38; $p=0.019$. The deviant grand average waves were systematically more negative in comparison with standard grand average waves (Figure $2 \mathrm{~A}$; dark grey rectan- 
gle). Analysis of the difference waves with a one-sampled t-test revealed significant differences from zero at all four frontocentral electrode sites, indicating a typical MMN evoked by deviant speech sounds in beginner readers: at Fz, $t(12)=-5.66 ; p<$ 0.001 , at $\mathrm{Cz}, t(12)=-4.91 ; p<0.001$, at FC3, $t(12)=-5.92 ; p<0.001$ and at FC4, $t(12)$ $=-5.38 ; p<0.001$.

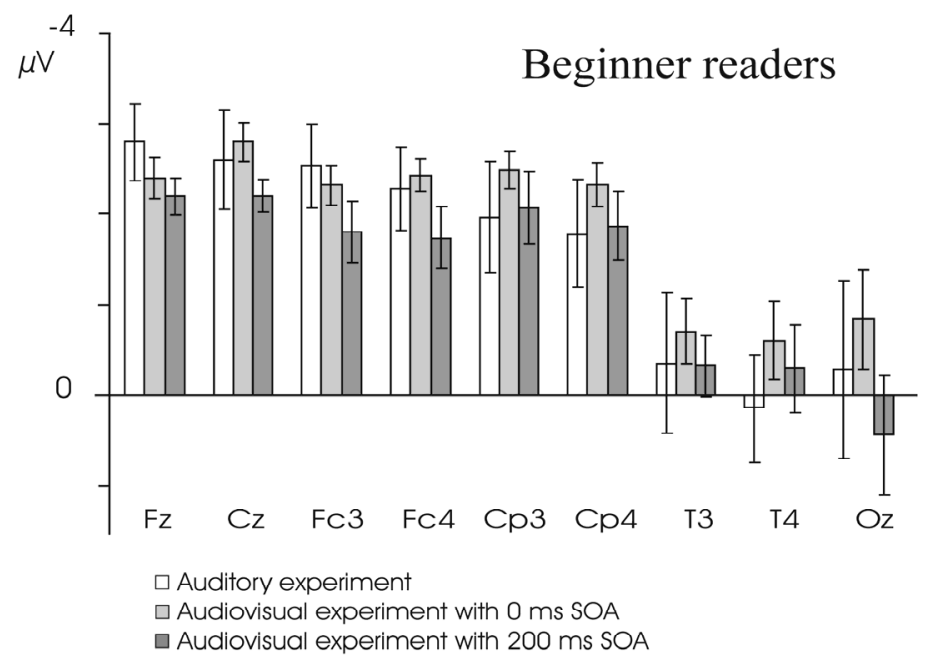

Figure 3. Mean amplitude values over $50 \mathrm{~ms}$ around the individually assessed $\mathrm{MMN}$ peak with standard error for all electrodes included in the analysis of beginner readers (Fz, Cz, FC3, FC4, Cp3, Cp4, T3, T4 and $\mathrm{Oz})$.

Auditory versus audiovisual experiments: In all 3 experiments (the auditory experiment and both audiovisual experiments $A V_{-} 0$ and $A V_{-} 200$ ) the deviant speech sound stimulus elicited a negativity with an average peak latency (averaged over the four frontocentral electrodes) at $165 \mathrm{~ms}$ (57 ms SD) after auditory stimulus onset (Figure 2; dark grey rectangle), with a typical topographical distribution of the MMN. Average peak latencies (averaged over the four frontocentral electrodes) were 156 ms (44 ms SD) for AV_0 and 181 ms (68 ms SD) for AV_200 after the onset of the auditory stimulus.

Amplitude and latency properties of ERP activity in the MMN time-window were first analyzed using a mixed ANOVA with two within-subject factors, stimulus type (2) and electrode sites (11), and one between group variable, experiment (3). We analyzed the responses reflecting stimulus mismatch. There was a significant interaction between stimulus type and electrode, $F(10,390)=3.57 ; p=0.010$. When the same analysis was performed on only the four frontocentral electrodes there is no significant interaction with electrodes, $F(3,117)=2.65 ; p=0.057$. There was no significant interaction between stimulus type and experiment, indicating compara- 
ble $M M N$-effects in each of the three experiments, $F(1,39)=2.61 ; p=0.086$. The MMN-effect was reflected by a significantly stronger negative ERP response for the deviant stimuli in comparison to the standard stimuli (main effect of stimulus type for mean amplitude, $F(1,39)=28.73 ; p<0.001)$.

Table 2. Peak latency and mean area amplitude measures on the four frontocentral electrodes with standard errors of the mean of beginner readers for the three experiments

\begin{tabular}{llll}
\hline Difference waves & \multicolumn{1}{c}{ Auditory } & Audiovisual 0 ms SOA & Audiovisual 200 ms SOA \\
\hline Peak latency \pm SE $(\mathrm{ms})$ & & \\
$\mathrm{Fz}$ & $164 \pm 15.3$ & $152 \pm 9.1$ & $186 \pm 21.1$ \\
$\mathrm{Cz}$ & $152 \pm 14.9$ & $157 \pm 12.3$ & $180 \pm 20.9$ \\
FC3 & $154 \pm 15.6$ & $158 \pm 12.8$ & $180 \pm 17.6$ \\
FC4 & $152 \pm 15.1$ & $159 \pm 13.3$ & $179 \pm 18.0$ \\
Mean area \pm SE $(\mu \mathrm{V})$ & & & \\
Fz & $-2.8 \pm 0.5$ & $-2.4 \pm 0.3$ & $-2.2 \pm 0.2$ \\
Cz & $-2.6 \pm 0.4$ & $-2.8 \pm 0.2$ & $-2.2 \pm 0.2$ \\
FC3 & $-2.6 \pm 0.4$ & $-2.3 \pm 0.2$ & $-1.7 \pm 0.3$ \\
FC4 & $-2.4 \pm 0.5$ & $-2.5 \pm 0.2$ & $-1.8 \pm 0.3$ \\
\hline
\end{tabular}

Table 2 shows the peak latency and mean area amplitude measures of the difference waves on the four frontocentral electrodes evoked by the auditory deviant in the auditory experiment and in both audiovisual experiments, AV_0 and AV_200. The latency values are reported with respect to the onset of the auditory stimulus. Repeated measures ANOVA on the latency of the MMN over the experiments did not show a significant effect on latency, $F<1$. Repeated measures ANOVA on amplitude of the difference waves of the frontocentral electrodes revealed no significant effect of experiment, $F(2,39)=1.32 ; p=0.279$ (Figure 3 ), indicating no differences in the $\mathrm{MMN}$ as evoked in the auditory experiment versus the audiovisual experiments. The difference waves revealed however an interesting difference between the three experiments at a negativity around $650 \mathrm{~ms}$ after auditory stimulus onset (Figure 2; light grey rectangle). The analysis of this negativity, referred to as the Late Negativity, is presented below. 


\section{Advanced readers}

\section{A. Auditory experiment}

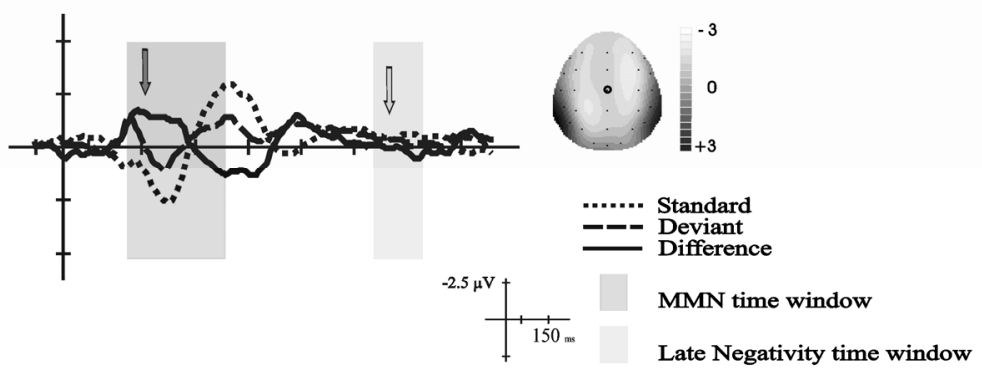

\section{B. Audiovisual experiment $0 \mathrm{~ms}$ SOA}
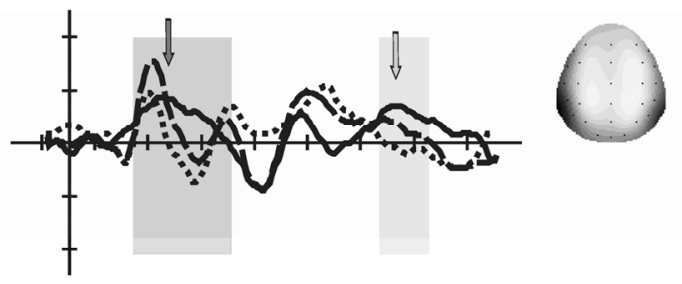

\section{Audiovisual experiment 200 ms SOA}

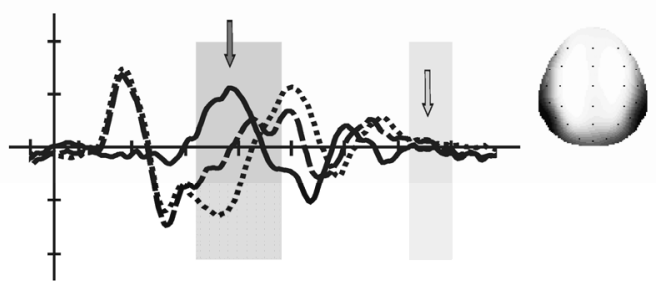

Figure 4. Grand average ERPs for the standard (dotted lines) and deviant stimuli (dashed lines), difference waves (solid lines) as measured at $C z$, and $M M N$ maps for the auditory experiment $(A)$ and the two audiovisual experiments $0 \mathrm{~ms}$ SOA (B) and $200 \mathrm{~ms}$ SOA (C) with advanced readers.

\section{Advanced readers}

Auditory experiment: We analyzed the auditory experiment to probe a typical MMN in advanced readers and compared these results with the MMN evoked by beginner readers. Figure 4A shows the grand average ERPs and difference waveforms (deviant - standard stimuli) for the speech sound deviant in the auditory experiment. The speech sound deviant elicited an MMN with average peak latency of 152 ms (averaged over the four frontocentral electrodes, $45 \mathrm{~ms}$ SD) after the onset of the audi- 
tory stimulus, with a topographical distribution (Figure 4A) typical for the MMN (Picton et al., 2000; Schröger, 1998).

Amplitude properties of brain waves in the MMN time-window were first analyzed using a 2 (stimulus type; standard versus deviant) by 11 (electrode sites) repeatedmeasures ANOVA. We found a significant interaction with electrode site, $F(10,110)$ $=10.74 ; p=0.001$ (Figure 5). When we analyzed only the four frontocentral electrodes there was no longer a significant interaction between stimulus type and electrode, $F(3,33)=2.41 ; p=0.098$. We found a main effect of stimulus type for mean amplitude values in the $50 \mathrm{~ms}$ window around the individual peaks, $F(1,11)=$ $26.65 ; p<0.001$. The deviant grand average waves were systematically more negative in comparison with standard grand average waves (Figure 4; dark grey rectangle). Analysis of the difference waves with a one-sampled t-test revealed significant differences from zero in all four frontocentral electrodes, indicating a typical MMN evoked by deviant speech sounds in advanced readers: at Fz, $t(11)=-5.84 ; p<$ 0.001 , at $\mathrm{Cz}, t(11)=-6.34 ; p<0.001$, at FC3, $t(11)=-3.51 ; p=0.005$ and at FC4, $t(11)$ $=-6.04 ; p<0.001$.

Auditory versus audiovisual experiments: In all 3 experiments (the auditory experiment and both audiovisual experiments, AV_0 and AV_200) the deviant speech sound stimulus elicited a negativity with an average peak latency (averaged over the four frontocentral electrodes) at $166 \mathrm{~ms}$ (41 ms SD) after auditory stimulus onset (Figure 4; dark grey rectangle), with a typical topographical distribution of the MMN. Average peak latencies (averaged over the four frontocentral electrodes) were $181 \mathrm{~ms}$ (42 ms SD) for AV_0 and $163 \mathrm{~ms}$ (33 ms SD) for AV_200 after the onset of the auditory stimulus. We first analyzed the responses reflecting stimulus mismatch with a repeated measures ANOVA with stimulus type (2) and electrode (11) as within subject factors and experiment (3) as a between subject factor. There was a significant interaction between stimulus type and electrode, $F(10,340)=15.95 ; p<$ 0.001 (Figure 5). The same analysis on only the four frontocentral electrodes revealed no significant interaction with electrodes $F(3,102)=1.35 ; p=0.27$. More importantly, there was a significant interaction between stimulus type and experiment, $F(2,34)=5.30 ; p=0.010$, indicating a different MMN over the three experiments. Despite this interaction the ERP response to the deviant stimuli was consistently more negative in comparison to the standard stimuli in all three experiments (main effect of stimulus type for mean amplitude $F(1,34)=108.62 ; p<0.001$ ). 


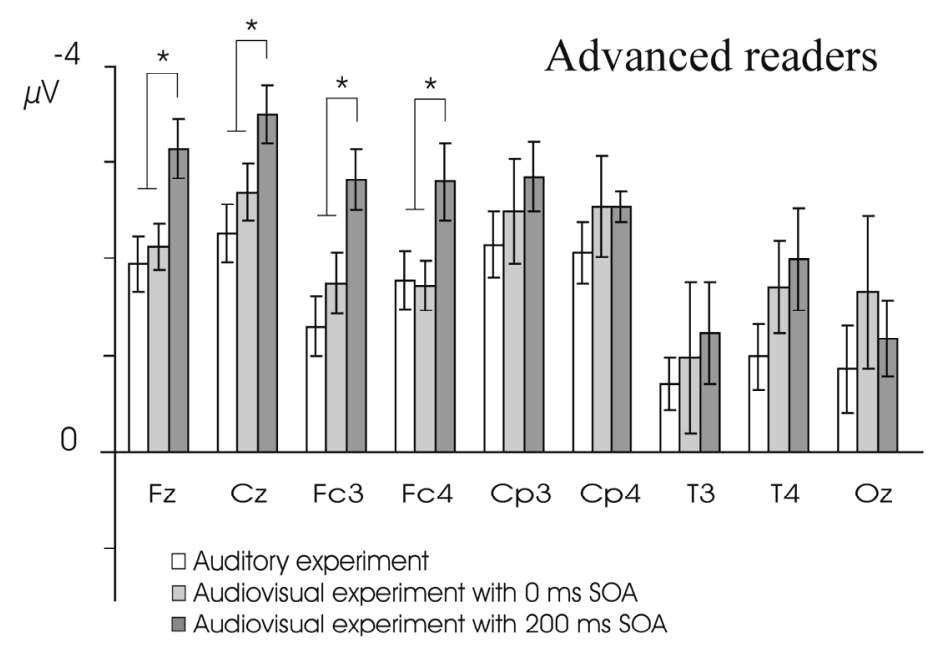

Figure 5. Mean amplitude values over $50 \mathrm{~ms}$ around the individually assessed MMN peak with standard error for all electrodes included in the analysis with advanced readers ( Fz, Cz, FC3, FC4, Cp3, Cp4, T3, T4 and $\mathrm{Oz}$ ). Significant differences $(p<0.05)$ in mean amplitude measures are indicated per electrode by an asterisk.

Table 3 shows the peak latency and mean area amplitude measures of the difference waves for the four frontocentral electrodes evoked by the auditory deviant in the auditory experiment and in both audiovisual experiments. The latency values are reported with respect to the onset of the auditory stimulus. Repeated measures ANOVA on the latency of the MMN over the experiments did not show a significant effect of latency $F(2,34)=1.51 ; p=0.235$. Repeated measures ANOVA on the mean of the four difference waves of the frontocentral electrodes revealed a marginally significant effect of experiment, $F(2,32)=3.24, p=0.052$. Since we had a-priori expectations about the direction of the differences between the experiments (enhancement of the MMN to the deviant speech sound when presented in the context of a letter in comparison with presentation in isolation), we calculated contrasts of the mean values of the MMN to analyze these differences in detail. In contrast with our expectations, planned contrasts revealed that the context of letters in AV_0 did not significantly increase the amplitude of the $M M N$ to the auditory deviant in comparison with no letter context $t(31)=1.046, p=0.324$. In AV_200 however the amplitude of the MMN to the auditory deviant significantly increased in comparison with the MMN amplitude in the auditory experiment, $t(31)=2.351, p=0.025$ (Fig $6 \mathrm{~B}$. left set of bar graphs). Contrasting the $M M N$ amplitude in $A V \_0$ with the MMN in $A V \_200$ revealed a marginally significant difference, $t(31)=2.005, p=0.053$ (Figure $6 \mathrm{~B}$, left set of bar graphs), indicating an effect of stimulus onset asynchrony (SOA) in the advanced readers. This effect of SOA is however reversed if compared with the SOA-effect in the experienced adult readers (Figure 6; dark grey rectangle) 
(Froyen et al., 2008). The difference waves also revealed an interesting difference between the three experiments at a negativity around $650 \mathrm{~ms}$ after auditory stimulus onset (Figure 4; light grey rectangle). The analysis of this negativity observed in beginner and advanced readers is presented below.

Table 3. Peak latency and mean area amplitude measures on the four frontocentral electrodes with standard errors of the mean of advanced readers for the three experiments

\begin{tabular}{llll}
\hline Difference waves & \multicolumn{1}{c}{ Auditory } & Audiovisual 0 ms SOA & Audiovisual 200 ms SOA \\
\hline Peak latency \pm SE $(\mathrm{ms})$ & & \\
$\mathrm{Fz}$ & $136 \pm 14.4$ & $178 \pm 13.3$ & $166 \pm 11.1$ \\
$\mathrm{Cz}$ & $146 \pm 14.1$ & $172 \pm 12.9$ & $163 \pm 9.5$ \\
FC3 & $148 \pm 15.6$ & $189 \pm 12.5$ & $161 \pm 10.9$ \\
FC4 & $151 \pm 13.0$ & $184 \pm 13.9$ & $162 \pm 11.9$ \\
Mean area \pm SE $(\mu \mathrm{V})$ & & & \\
Fz & $-2.2 \pm 0.4$ & $-2.3 \pm 0.3$ & $-3.1 \pm 0.2$ \\
Cz & $-2.6 \pm 0.4$ & $-2.8 \pm 0.3$ & $-3.5 \pm 0.3$ \\
FC3 & $-1.4 \pm 0.3$ & $-1.8 \pm 0.3$ & $-2.8 \pm 0.3$ \\
FC4 & $-2.0 \pm 0.4$ & $-1.9 \pm 0.3$ & $-2.8 \pm 0.4$ \\
\hline
\end{tabular}

Stable auditory MMN early in development

To ascertain that both beginner and advanced readers elicited a comparable MMN in the auditory experiment we performed a one-way ANOVA comparing the difference waves of the beginner readers with the difference waves of the advanced readers. This analysis revealed no significant differences considering mean or latency area value of the MMN, $F(2,24)=1.49, p=0.246$ and $F<1$, respectively. We also performed a one-way ANOVA comparing the difference waves of the beginner readers and the advanced readers with the difference waves of the experienced adult readers from our earlier study (Froyen et al., 2008). This analysis revealed no significant differences for the mean or latency values of the MMN (Figure 6; dark grey rectangle) $F<1$ and $F<1$, respectively, indicating a stable auditory MMN resembling the adult MMN already early in development.

\section{Late Negativity}

The difference waves revealed another interesting difference between the three experiments around $650 \mathrm{~ms}$ after auditory stimulus onset in both the beginner and advanced readers (Figure 6; light grey rectangle). We therefore tested this negativity statistically within each age group with a repeated measures ANOVA, with 4 frontocentral electrodes (Fz, Cz, FC3 and FC4) as a within subject factor and experiment as between subject factor. The dependent variable was the mean of $50 \mathrm{~ms}$ around the peak values between 600 and $700 \mathrm{~ms}$ after auditory stimulus onset, established per subject for each electrode in each experiment. 
In the beginner readers there was a marginally significant main effect of experiment, $F(2,39)=3.235 ; p=0.050$. Post hoc t-tests (Tukey corrected) revealed a significant difference between the auditory and the audiovisual experiment AV_200, $t(39)=1.215, p=.045$, but no significant differences between the auditory and audiovisual experiment $A V \_0, t(39)=.4083, p=.693$, or between both audiovisual experiments $A V \_0$ and $A V \_200, t(39)=.8069, p=.226$. Apparently, at $650 \mathrm{~ms}$ after speech sound onset, there is an influence of letter context on speech sound processing in beginner readers, but only when the letter is presented $200 \mathrm{~ms}$ before the speech sound. In the advanced readers we also found a main effect of experiment, $F(2,31)=3.562 ; p=0.038$. Post hoc t-tests (Tukey corrected) revealed a significant difference between the auditory experiment and the audiovisual experiment AV_0, $t(31)=2.074, p=.031$, and no differences between the auditory experiment and AV_200, $t(31)=1.361, p=.188$, or between AV_0 and AV_200, $t(31)=.7131, p=$ 0.632 . Advanced readers show an influence of letter context on speech sound processing around $650 \mathrm{~ms}$ after auditory speech sound onset, but unlike beginner readers only when the letter is presented simultaneously with the speech sound.

The negativity described here probably does not reflect the late mismatch negativity or IMMN as described in earlier studies (Cheour, Korpilahti, Martynova, \& Lang, 2001; Korpilahti, Krause, Holopainen, \& Lang, 2001). The IMMN is also revealed in the present data between 450 and 500 ms after auditory stimulus onset (Figure 6; white rectangle), a typically reported latency (Cheour et al., 2001; Korpilahti et al., 2001). We tested the IMMN statistically within each age group with a repeated measures ANOVA, with 4 frontocentral electrodes (Fz, Cz, FC3 and FC4) as a within subject factor and experiment as between subject factor. The dependent variable was the mean of $50 \mathrm{~ms}$ around the peak values between 400 and $500 \mathrm{~ms}$ after auditory stimulus onset, established per subject for each electrode in each experiment. Neither the beginner readers nor the advanced readers revealed an effect of experiment in this time window, $F(2,39)=1.211 ; p=0.309$ and $F<.1$, respectively. 


\section{Difference waves}

A) beginner readers

B) Advanced readers

C) Adult readers

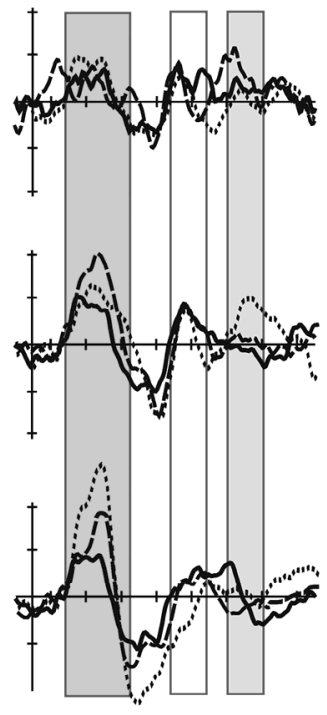

MMN time-window

IMMN time-window

Late negativity time-window

\section{Mean amplitude values}
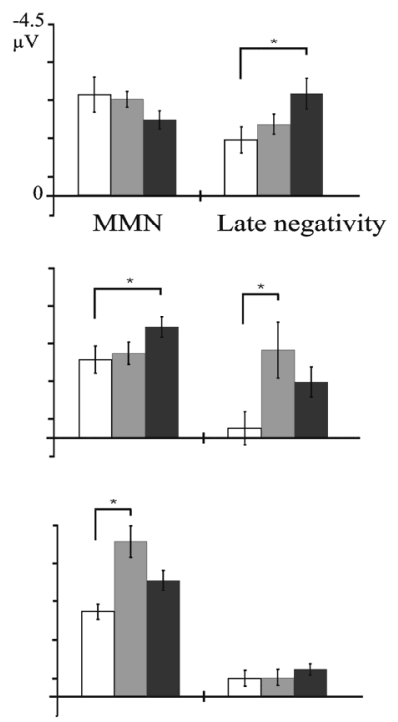

$\square$ Auditory experiment

AV $0 \mathrm{~ms}$

AV $200 \mathrm{~ms}$

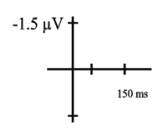

Figure 6. Difference waves on the frontocentral electrode $\mathrm{Cz}$ as measured in the auditory and the audiovisual experiments for beginner, advanced (this study) and experienced adult readers (Froyen, Van Atteveldt, Bonte, \& Blomert, in press) with the different time-windows of the MMN, the late mismatch negativity (IMMN) and the Late Negativity indicated in rectangles (1). Mean amplitude values with standard error for the MMN (left) and the Late Negativity (right) averaged over the four frontocentral electrodes Fz, Cz, FC3 and FC4 for beginner, advanced (this study) and experienced adult readers (Froyen et al., in press) (2). Significant differences $(p<0.05)$ are indicated with an asterisk. 


\section{Discussion}

An MMN - paradigm was used to study the development of letter - speech sound integration. The influence of letters on speech sound processing was investigated in beginner and advanced readers. In order to investigate the temporal window for letter - speech sound processing during development, we manipulated stimulus onset asynchrony (SOA). The letter was presented either simultaneously with the speech sound or $200 \mathrm{~ms}$ before the speech sound. The data revealed unexpected results at an MMN latency around $150 \mathrm{~ms}$ and at a later latency around $650 \mathrm{~ms}$. In order to assure a standard MMN in all age groups we compared the MMN evoked to speech sounds in the auditory experiment with beginner, advanced and also experienced adult readers (Froyen et al., 2008). All groups elicited a typical MMN in terms of amplitude, latency and topographical distribution (Picton et al., 2000; Schröger, 1998). Moreover, no differences were found in amplitude, latency or topographical distribution between the groups, which confirmed the suggestion of a stable $\mathrm{MMN}$ resembling the adult $\mathrm{MMN}$ being already present early in development (Csépe, 2003).

Next, the influence of letters on speech sound processing was investigated in beginner readers. Comparing the $\mathrm{MMN}$ in the auditory experiment with the $\mathrm{MMN}$ in the audiovisual experiments revealed no differences in amplitude, latency or topographical distribution. This indicates that after approximately one year of reading instruction letters do not influence early speech sound processing and thus there is no indication of any form of automaticity of the letter - speech sounds processing. This does not seem to be consistent with the current educational Dutch standards requiring full mastery of letter - speech sound associations within one year of reading instruction (Wentink \& Verhoeven, 2003). These results therefore reveal that fully developed letter - speech sound processing requires more than just accurate association. Consistent with the reaction time data reported in Blomert \& Vaessen (2009), these results point to an extended period of development which continues after this first year of reading instruction.

In advanced readers, comparison of the $\mathrm{MMN}$ in the auditory experiment with the MMN in the audiovisual experiments revealed a cross-modal enhancement of the MMN amplitude, but only when letters were presented 200 ms earlier in comparison with the speech sounds (AV_200). Since the MMN is an early and automatically evoked ERP-component, we suggest that early speech sound processing in advanced readers is automatically influenced by the presentation of a letter. However, advanced readers exhibited no differences in MMN and thus no sign of integration when the letter was presented simultaneously with the speech sound (AV_0). This contrasts sharply with the results from experienced adult readers only showing automatic integration if letters and speech sounds were presented simultaneously, and not if presented at 100 or 200 ms SOA (Froyen et al., 2008). Advanced readers show an influence of letters on the processing of speech sounds, but in a different 
temporal window for integration than experienced adult readers, indicating that even after four years of reading instruction letter - speech sound processing continues to develop.

Although the auditory experiment differs in visual aspects from the audiovisual experiments, it is unlikely that these differences are responsible for the present results. The $\mathrm{MMN}$ is known to be a very basic auditory deviance detection mechanism of the brain (Schröger, 1998). The similarity in morphology, latency and topographical distribution of the MMNs in the auditory and audiovisual experiments in both age groups suggests a similar underlying neural mechanism. Moreover, the difference in amplitude, as the measure of interest, seems not explainable by the differences in visual aspects between the auditory and the audiovisual experiment. Considering the advanced readers: the MMN evoked in AV_0 did not differ in any way, with the MMN evoked in the auditory experiment, despite differences in visual aspects between the two experiments. Furthermore, the $\mathrm{MMN}$-amplitude in AV_200 was significantly higher in comparison with the MMN-amplitude in AV_0. Visual aspects could not have affected this difference in MMN amplitude, since visual aspects were identical in both audiovisual experiments.

Interestingly, we also observed a noticeable pattern of amplitude enhancement of the difference waves at $650 \mathrm{~ms}$ after speech sound onset (Figure 6). As described in the results, we distinguish the negativity observed at $650 \mathrm{~ms}$ from the late mismatch negativity or IMMN described in earlier studies (Cheour et al., 2001; Korpilahti et al., 2001). The IMMN is evoked between 400 and 450 ms after auditory stimulus onset, which is earlier in comparison with the negativity considered here. Because the exact nature of the negativity observed at $650 \mathrm{~ms}$ is as yet unclear we will refer to it as "Late Negativity". In combination with the results from the MMN, the pattern of the Late Negativity amplitudes in beginner and advanced readers revealed a complex but systematic development of letter - speech sound processing. The beginner readers exhibited an enhancement of the Late Negativity amplitude in AV_200 in comparison with the amplitude of the Late Negativity in the auditory experiment (Figure 6A; light grey rectangle). Remarkably, the observed trend of Late Negativity amplitudes in the beginner readers resembles the pattern of MMN amplitudes in the advanced readers: enhancement of the MMN amplitude in AV_200 in comparison with the MMN amplitude in the auditory (Figure 6B; dark grey rectangle). So while for beginner readers there is no indication of an influence of the letter 'early' during speech sound processing, beginner readers do reveal an influence of the letter 'later' during speech sound processing, at SOA_200. In the advanced readers enhancement of the Late Negativity amplitude was revealed in the audiovisual experiment $A V_{-} 0$ in comparison with the Late Negativity amplitude in the auditory experiment (Figure 6B; light grey rectangle). The observed pattern of Late Negativity amplitudes in the advanced readers resembles the pattern of MMN 
amplitudes in the experienced adult readers remarkably well (Froyen et al., 2008) with an enhancement of the MMN amplitude in AV_O in comparison with the MMN amplitude in the auditory experiment (Figure 6C; dark grey rectangle). The observed pattern of Late Negativity amplitudes indicates 'adult-like' letter - speech sound processing in advanced readers, however at a later latency; 650 ms instead of 150 ms after speech sound onset.

Considering the changes in MMN and in Late Negativity amplitudes, there seems to be a double shift in timing properties during the development of letter - speech sound processing, both in the latency at which letter - speech sound processing occurs and in the temporal window of integration (i.e. SOA) itself. Data from our previous study reveal early and automatic integration in the experienced adult readers only if letters and speech sounds were presented simultaneously (Figure 6C) (Froyen et al., 2008). In contrast, the beginner readers show no sign of integration but only a late influence of letters on speech sound processing; around $650 \mathrm{~ms}$ after presentation of the speech sounds, and only at an SOA of $200 \mathrm{~ms}$ between the presentation of letters and speech sounds (Figure 6A). The advanced readers show early and automatic integration around $150 \mathrm{~ms}$, however only at an SOA of $200 \mathrm{~ms}$ (Figure 6B), which is still at a different temporal window for integration in comparison with the experienced adult readers. Advanced readers showed a more 'adultlike' temporal window for integration, when letters and speech sounds were presented simultaneously but not with a 200 ms SOA, This effect, however, was only seen $650 \mathrm{~ms}$ after auditory stimulus onset (Figure 6B). This double shift in timing properties may be interpreted as a neural fine-tuning of letter - speech sound processing extending far beyond the early school years, most likely caused by continued brain maturation interacting with increasing reading experience. Continuing pruning and myelination in the temporal lobes, containing speech related areas, indicates brain maturation beyond adolescence (Casey, Tottenham, Liston, \& Durston, 2005; Sowel et al., 2003). At the same time the brain is subject to rapidly increasing reading experience from the first school year on.

The shift in SOA from 200 to 0 ms indicates a shift of the temporal window of letter - speech sound integration over development. This is consistent with the observation of changing temporal windows for processing audiovisual stimuli from childhood to adulthood (Laasonen et al., 2000; 2001).

The shift in latency at which letter - speech sound processing occurs, namely from $650 \mathrm{~ms}$ after speech sound onset in beginner and advanced readers to $150 \mathrm{~ms}$ after speech sound onset in advanced and experienced adult readers (Froyen et al., 2008), does not seem to be an effect of straightforward brain maturation. It is generally assumed that ERP-response latencies decrease with increasing age (Bonte et al., 2007; Brem et al., 2006; Maurer et al., 2003; Shafer et al., 2000). Brem et al. (2006) relate decreasing latencies to increases in processing speed as a conse- 
quence of general brain maturation. However, the above mentioned ERP-studies always report a decrease in latency within one and the same ERP-component, respectively the MMN (Bonte et al., 2007; Maurer et al., 2003; Shafer et al., 2000) or the N1 (Brem et al., 2006). In contrast, the present study shows no differences in the latency of the MMN, neither between younger and older children nor between children and adults, The time shift does not refer to a different latency of the same process at a different age, but probably reflects a shift between different mechanisms responsible for letter - speech sound processing in beginner, advanced and adult readers. Additionally, letter - speech sound processing in beginner readers, as reflected in the Late Negativity, occurs no less than $\mathbf{5 0 0}$ ms later in comparison with fully developed letter - speech sound processing. The observed latency shift is much larger than reported in any of the above mentioned ERP-studies and thus is unlikely to be caused by the continued decrease in processing time of the maturing brain alone. We therefore suggest that rapidly increasing experience with reading during the first years at school may affect, besides processing speed, also the nature of letter - speech sound processing. Whereas beginner readers may merely associate letters to speech sounds, increasing experience with reading may lead to automatic letter - speech sound integration. Once letter - speech sound integration is established, letter - speech sound processing by mere association becomes redundant, as reflected in a total disappearance of the Late Negativity effect in experienced adult readers (Froyen et al., 2008). The fact that the late negativity pattern of beginner readers (Figure 6A) resembles the early pattern in advanced readers (Figure 6B) and the late negativity pattern in advanced readers (Figure $6 B$ ) resembles the early pattern in adults (Figure $6 \mathrm{C}$ ) underpins our suggestion. The advanced readers seem to be able to use both mechanisms depending on SOA; integration seems possible at $200 \mathrm{~ms}$ SOA (early pattern), but the simultaneous presentation of letters and speech sounds (effective for adults) still seems too far fetched at this age and level of reading expertise and invokes the late association processes (late pattern), also observable in the beginner readers.

This double shift in timing properties of letter-speech sound processing during development may provide an explanation for the seemingly inconsistent results from behavioral studies. While accurate letter - speech sound associations are established within one year of reading instruction in Dutch (Blomert \& Vaessen, 2009; Wentink \& Verhoeven, 2003), reaction time data suggest protracted development beyond the early school years (Blomert \& Vaessen, 2009). The present results show that the processes associating letters with speech sounds occur late in time, i.e. not earlier than 650 ms after auditory stimulus onset. In contrast, adult readers recruit only very early processes within $\mathbf{2 0 0}$ milliseconds after stimulus onset. These findings are consistent with longer reaction times, but at the same time do not contradict maximal accuracy already after one year of reading instruction. In sum, accuracy alone does not appear to be a sensitive indicator for letter - speech sound 
integration. The present brain findings are more consistent with the reaction time results, both strongly indicating protracted development of letter - speech sound associations far beyond early school years.

In our previous $\mathrm{fMRI}$ studies investigating letter - speech sound processing (Van Atteveldt et al., 2004, 2007a, 2007b), we have used many different single letters and speech sounds (vowels: a, o, i, e, u, y, and consonants: $d, g, h, k, l, n, p, r, s, t, z$ ). We have always checked the results for the different stimuli separately and our consistent observation was that all single letters/speech sounds evoked the same activation patterns and integration effects. Therefore, we believe our present selection of "a" and "o" is reliably representative and consequently generalizable to other letter - speech sound pairs.

In conclusion, despite proper knowledge of the associations between letters and their corresponding speech sounds, beginner readers have not developed early and automatic integration yet. Advanced readers exhibited early and automatic integration of letters and speech sounds, however still at a different temporal window of integration than adults. A more 'adult-like' pattern is only observed hundreds of milliseconds later, indicating that even after four years of reading instruction letter - speech sound integration still continues to develop. The differences in temporal windows of integration and the shifts in latency of letter - speech sound processing probably reflect a double shift in timing properties during the development of letter - speech sound processing. Continued brain maturation and reading experience most likely contribute interactively to a neural fine-tuning of letter - speech sound processing during the development towards early and automatic letter - speech sound integration.

\section{Acknowledgments}

This work was supported by grant 608/022/2005 of the Dutch Board of Health Care Insurance (College voor Zorgverzekeringen) awarded to L.B. We thank three anonymous Reviewers for their constructive remarks that helped us to improve the paper and Martin Frost for final editing. We thank Hanne Poelmans for assistance in data acquisition. Special thanks goes to the participating children and the schools "Aloysius" and "De Perroen" from Maastricht for their cooperation.

\section{References}

Blomert, L., \& Vaessen, A. (2009). Dyslexie Differentiaal Diagnose; Cognitief onderzoek van lezen en spellen [Dyslexia differential Diagnosis; cognitive investigation of reading and spelling]. Amsterdam: Boom B.V. publishers. 
Boersma, P., \& Weenink, D. (2002). Praat 4.0: a system for doing phonetics with the computer. Amsterdam: Universiteit van Amsterdam.

Bonte, M., Poelmans, H., \& Blomert, L. (2007). Deviant neurophysiological responses to phonological regularities in speech in dyslexic children. Neuropsychologia, 45, 1427-1437.

Brem, S., Bucher, K., Halder, P., Summers, P., Dietrich, T., Martin, E., et al. (2006). Evidence for developmental changes in the visual word processing network beyond adolencence. Neuroimage, 29, 822 837.

Brus, B. T., \& Voeten, M. J. M. (1973). Een-minuuttest, vorm A en B. Nijmegen: Berkhout.

Calvert, G. A., Brammer, M. J., \& Iversen, S. D. (1998). Crossmodal identification. Trends in Cognitive Sciences, 2, 247-253.

Casey, B. J., Tottenham, N., Liston, C., \& Durston, S. (2005). Imaging the developing brain: what have we learned about cognitive development? Trends in Cognitive Sciences, 9, 104-110.

Cheour, M., Korpilahti, P., Martynova, O., \& Lang, A. H. (2001). Mismatch negativity and late discriminative negativity in investigating speech perception and learning in children and infants. Audiology and Neuro-otology, 6, 2-11.

Csépe, V. (2003). Dyslexia: different Brain, Different Behavior. New York: Kluwer Academic/ Plenum Publishers.

Dijkstra, T., Schreuder, R., \& Frauenfelder, U. H. (1989). Grapheme Context Effects on Phonemic Processing. Language and Speech, 32, 89-108.

Ehri, L. C. (2005). Development of sight word reading: phases and findings. In M. J. Snowling \& C. Hulme (Eds.), The science of reading: A handbook (pp. 135 - 145). Oxford: Blackwell Publishing.

Fox, E. (1994). Grapheme-phoneme correspondence in dyslexic and matched control readers. British Journal of Psychology, 85, 41-53.

Frith, U. (1985). Beneath the surface of developmental dyslexia. In K. E. Patterson, J. C. Marshall \& M. Coltheart (Eds.), Surface dyslexia. London: Routledge \& Kegan-Paul.

Froyen, D., van Atteveldt, N., Bonte, M., \& Blomert, L. (2008). Cross-modal enhancement of the MMN to speech sounds indicates early and automatic integration of letters and speech sounds. Neuroscience Letters, 430, 23-28.

Froyen, D., Van Atteveldt, N., Bonte, M., \& Blomert, L. (in press). Cross-modal enhancement of the MMN to speech sounds indicates early and automatic integration of letters and speech sounds. Neuroscience Letters.

Hardy, M. H., Smythe, P. C., Stennet, R. G., \& Wilson, H. R. (1972). Developmental patterns in elemental reading skills: phoneme-grapheme and grapheme-phoneme correspondences. Journal of Educational Psychology, 63, 433-436.

Herdman, A. T., Fujioka, T., Chau, W., Ross, B., Pantev, C., \& Picton, T. W. (2006). Cortical oscillations related to processing congruent and incongruent grapheme-phoneme pairs. Neuroscience Letters, 399, $61-66$.

Kasai, K., Nakagome, K., Iwanami, A., Fukuda, M., Itoh, K., Koshida, I., et al. (2002). No effect of gender on tonal and phonetic mismatch negativity in normal adults assessed by a high resolution EEGrecording. Cognitive Brain Research, 13, 305-312.

Korpilahti, P., Krause, C. M., Holopainen, I., \& Lang, A. H. (2001). Early and late mismatch negativity elicited by words and speech-like stimuli in children. Brain and Language, 76, 332-339.

Laasonen, M., Service, E., \& Virsu, V. (2002). Crossmodal temporal order and processing acuity in developmentally dyslexic young adults. Brain and Language, 80, 340-354.

Laasonen, M., Tomma-Halme, J., Lahti-Nuuttila, P., Service, E., \& Virsu, V. (2000). Rate of information segregation in developmentally dyslexic children. Brain and Language, 75, 66-81.

Maurer, U., Bucher, K., Brem, S., \& Brandeis, D. (2003). Development of the automatic mismatch response: from frontal positivity in kindergarten children to the mismatch negativity. Clin Neurophysiol, 114, 808-817.

Mitterer, H., \& Blomert, L. (2003). Coping with phonological assimilation in speech perception: evidence for early compensation. Percept Psychophys, 65, 956-969. 
Näätänen, R. (2000). Mismatch negativity (MMN): perspectives for application. Int J Psychophysiol., 37, 3-10.

Näätänen, R. (2001). The perception of speech sounds by the human brain as reflected by the mismatch negativity (MMN) and its magnetic equivalent (MMNm). Psychophysiology, 38, 1-21.

Näätänen, R., Paavilainen, P., Tiitinen, H., Jiang, D., \& Alho, K. (1993). Attention and mismatch negativity. Psychophysiology, 30, 436-350.

Nuwer, M., Comi, G., Emerson, R., Fuglsang-Frederiksen, A., Guérit, J., Hinrichs, H., et al. (1998). IFCN standards for digital recording of clinical EEG. Electroencephalogr Clin Neurophysiol, 106, 259-261.

Picton, T. W., Alain, C., Otten, L., Ritter, W., \& Achim, A. (2000). Mismatch negativity: different water in the same river. Audiol Neurootol, 5, 111-139.

Raij, T., Uutela, K., \& Hari, R. (2000). Audiovisual integration of letters in the human brain. Neuron, 28, 617-625.

Raven, J., Raven, J.C., Court, J.H. (1998). Coloured progressive matrices. Oxford: Oxford Psychologists Press Ltd.

Schneider, W., \& Chein, J. M. (2003). Controlled \& automatic processing: behavior, theory and biological mechanisms. Cognitive Science, 27, 525-559.

Schröger, E. (1998). Measurement and interpretation of the mismatch negativity. Behavior Research Methods Instruments \& Computers, 30, 131-145.

Semtlitsch, H., Anderer, P., Schuster, P., \& Presslich, O. (1986). A solution for reliable and valid reduction of ocular artefacts, applied to the P300 ERP. Psychophysiology, 23, 695-703.

Shafer, V. L., Morr, M. L., Kreuzer, J. A., \& Kurtzberg, D. (2000). Maturation of mismatch negativity in school-age children. Ear and Hearing, 21, 242-251.

Siegel, L. S., \& Faux, D. (1989). Aquisition of certain grapheme-phoneme correspondences in normally achieving and diabled readers. Reading and Writing, an interdisciplinary journal, 1, 37-52.

Snowling, M. J. (1980). The development of grapheme-phoneme correspondence in normal and dyslexic readers. Journal of Experimental Child Psychology, 29, 294-305.

Sowel, E. R., Peterson, B. S., Thompson, P. M., Welcome, S. E., Henkenius, A. L., \& Toga, A. W. (2003). Mapping cortical change across the human life span. Nature Neuroscience, 6, 309-315.

Van Atteveldt, N., Formisano, E., Blomert, L., \& Goebel, R. (2007). The effect of temporal asynchrony on the multisensory integration of letters and speech sounds. Cerebral Cortex, 13, 962-974.

Van Atteveldt, N., Formisano, E., Goebel, R., \& Blomert, L. (2004). Integration of letters and speech sounds in the human brain. Neuron, 43, 271-282.

Van den Bos, K. P., Lutje Spelberg, H. C., Scheepsma, A. J. M., \& De Vries, J. R. (1999). De Klepel: pseudowoordentest: Lisse: Swets \& zeitlinger.

Van Wassenhove, V., Grant, K. W., \& Poeppel, D. (2007). Temporal window of integration in auditoryvisual speech perception. Neuropsychologia, 45, 598-607.

Vellutino, F. R., Fletcher, J. M., Snowling, M. J., \& Scanlon, D. M. (2004). Specific reading disability (dyslexia): what have we learned in the past four decades? J Child Psychol Psychiatry, 45(1), 2-40.

Wentink, H., \& Verhoeven, L. (2003). Protocol leesproblemen en dyslexie. Nijmegen: Expertisecentrum Nederlands.

Winkler, I., Kujala, A., Tiitinen, H., Sivonen, P., Alku, P., Lehtokoski, A., et al. (1999). Brain responses reveal the learning of foreign language phonemes. psychophysiology, 36, 638-642.

Yabe, H., Asai, R., Hiruma, T., Sutoh, T., Koyama, S., Kakigi, R., et al. (2004). Sound perception affected by nonlinear variation of accuracy in memory trace. Neuroreport, 15, 2813-2817.

Yabe, H., Tervaniemi, M., Sinkkonen, J., Huotilainen, M., Ilmoniemi, R., \& Naatanen, R. (1998). Temporal window of integration of auditory information in the human brain. Psychophysiology, 35, 615-619. 
Chapter 4

\section{Evidence for a specific cross-modal association deficit in dyslexia: An MMN- study of letter - speech sound processing} in dyslexia: An MMN-study of letter - speech sound processing. 


\begin{abstract}
The phonological deficit theory of dyslexia explains the reading problems by assuming that degraded speech sound representations hamper the acquisition of stable letter - speech sound associations necessary for learning to read. While there is abundant evidence for phonological problems in dyslexia there is only scarce evidence supporting the intermediate letter - speech sound association problem which assumedly causally connects phonological deficits to reading deficits. The present study aimed at clarifying the nature and the role of these assumed letter - speech sound association problems in dyslexia by analyzing the mismatch negativity (MMN) of eleven year old dyslexic children to speech sounds in isolation or combined with letters.

Recent studies with normal readers revealed that letters systematically modulated speech sound processing in an early time-window. The dyslexic readers in the present study, however, did not exhibit any early influences of letters on speech sounds even after four years of reading instruction, indicating no automatic integration of letters and speech sounds. Interestingly, they revealed a systematic late negativity to letter - speech sound pairs, probably indicating the mere association of letters and speech sounds. This pattern is strongly diverging from that observed in eleven year old normal readers but reminiscent of that observed in eight year old normal readers in a previous study. Additionally, dyslexic readers revealed normal processing of the same speech sounds, if these were presented without letters. Together these results point to a generic and specific cross-modal association deficit as the proximal cause of reading problems in dyslexia.
\end{abstract}




\section{Introduction}

Developmental dyslexia is a specific learning disability, characterized by reading and/or writing skills that are below what is predicted from their age and cognitive abilities (Lyon, Shaywitz, \& Shaywitz, 2003). The most commonly accepted cause for these reading problems is a phonological deficit mainly expressed in poor speech sound representations (Vellutino, Fletcher, Snowling, \& Scanlon, 2004; Ziegler \& Goswami, 2005). Although these two recent reviews show that there is abundant evidence for reading and concomitant phonological problems in dyslexia, the phonological deficit theory falls short of explaining how these evident phonological problems actually are causing the equally evident reading problems. This theoretical gap seems narrowed by the widely quoted belief (see the reviews above) that the assumedly degraded phonological representations hamper the acquisition of stable letter - speech sound associations. This is an intuitively appealing interpretation, since learning the correspondences between letters and speech sound is a prerequisite for the development of reading and writing skills in alphabetic orthographies (Ehri, 2005; Frith, 1985), and dyslexics have indeed been shown to experience problems in mastering letter - speech sound conversion rules (Fox, 1994; Siegel \& Faux, 1989; Snowling, 1980). However, these studies mainly used pseudoword reading to infer letter - speech sound problems, thereby potentially confounding letter speech sound knowledge with visual word recognition skills; e.g. in the Fox (1994) study, dyslexics performed surprisingly well on the visual word matching task, despite being poor readers. The problem therefore resides in the scarce direct empirical support for this crucial theoretical assumption bridging the gap between phonological and reading deficits. Moreover, most of the studies investigating letter speech sound knowledge were conducted in English, an orthography notorious for its opaque relations between letters and speech sounds and not representative for most other alphabetic languages (Share, 2008). For example, in a fairly transparent language like Dutch, most children know all letters and their corresponding speech sounds within one year of reading instruction (Aarnoutse, van Leeuwe, \& Verhoeven, 2000); even if they are at familial risk for dyslexia (Willems, Poelmans, Richardson, \& Blomert, submitted). In contrast, children learning the English orthography only fully mastered most letter - speech sound correspondences after several years of reading instruction (Hardy, Smythe, Stennet, \& Wilson, 1972; Siegel \& Faux, 1989) with still increasing mastery in grade eleven of secondary school (Calfee, Venezky, \& Chapman, 1969). A recent developmental study conducted in the relatively transparent Dutch language extended these findings by directly addressing letter - speech sound processing in a large sample of primary school dyslexic and non-impaired readers ranging from grade one to six (Blomert \& Vaessen, 2009). The children were tested for accuracy ánd speed of letter - speech sound identification and discrimination. Accuracy data indicated that it took till age eleven (grade 5) before children with dyslexia processed the associations between letters and 
speech sounds equally well as non-impaired age matched readers. Speed of letter speech sound identification and discrimination performance, however, was slower than that of non-impaired readers in all grades up to the age of twelve and did not decrease significantly beyond age nine. Thus, dyslexic readers do show significant impairments in processing letter - speech sound associations and seem to do so over many years of reading development. It should however be noted that nonimpaired readers also did show a continuous development in the speed with which letter - speech sound correspondences are processed; i.e. speed of letter - speech sound discrimination, corrected for normal motor speed development, decreased significantly up to grade six (Blomert \& Vaessen, 2009). These findings sharply contrast with the generally accepted assumption, that (in transparent orthographies) children accurately learn the associations between letters and speech sounds within months (Ziegler \& Goswami, 2005). It therefore seems necessary to make a distinction between acquiring declarative knowledge of letter - speech sound relations and the automating of letter - speech sound processing. Only the last process may be relevant for explaining dyslexia, since dyslexic, like non-impaired, readers displayed letter knowledge mastery within one year of reading instruction, but did show slow and less accurate letter - speech sound processing (Blomert \& Vaessen, 2009). Together these findings lead to the question: What is the nature of the letter - speech processing problem in dyslexia and what is its role in the development of reading problems?

Recently our insight in letter - speech sound processing in non-impaired readers has increased dramatically by several brain-imaging studies revealing the contours of a brain circuit for letter - speech sound processing in experienced readers (Blau, van Atteveldt, Formisano, Goebel, \& Blomert, 2008; Hashimoto \& Sakai, 2004; Raij, Uutela, \& Hari, 2000; Van Atteveldt, Formisano, Blomert, \& Goebel, 2007; Van Atteveldt, Formisano, Goebel, \& Blomert, 2004) and dyslexic readers (Blau, Atteveldt, Ekkebus, Goebel, \& Blomert, 2009). Interestingly, the time window for integration of letters and speech sounds was rather narrow; in normal adult readers, letters and speech sounds were successfully integrated only if the stimuli were presented simultaneously (Van Atteveldt, Formisano, Blomert et al., 2007). The evidence from these studies converges on heteromodal areas in the Superior Temporal Sulcus (STS) as a crucial locus for letter - speech sound processing. Van Atteveldt and colleagues furthermore revealed that not only heteromodal areas in STS, but also early auditory areas (Heschl's sulcus and Planum Temporale) were involved in letter speech sound integration. These results led to the proposal of the following neural mechanism for letter - speech sound processing in experienced readers: Sensory specific letter and speech sound input in early sensory areas not only converge but integrate in STS, immediately followed by feedback to "sensory specific" auditory areas signaling the adequacy of this letter - speech sound integration process (Blau 
et al., 2008; Van Atteveldt et al., 2004; Van Atteveldt, Formisano, Goebel, \& Blomert, 2007).

To investigate the automaticity and time characteristics of this letter - speech sound integration process we conducted an event related potentials (ERP) study in which a pre-attentive component, the mismatch negativity (MMN), was employed (Froyen, van Atteveldt, Bonte, \& Blomert, 2008). The MMN is an automatic auditory deviance detection mechanism with a major source located in the auditory cortex (Alho, 1995; Giard, 1990). It is evoked between 100 and 250 ms after stimulus onset when in a sequence of auditory stimuli a rarely presented sound (the deviant) deviates in one or more aspects from a frequently presented sound (the standard) (Näätänen, 1995; Schröger, 1998). A similar sensitivity of the MMN was demonstrated for language specific phonemic processes (Bonte, Mitterer, Zellagui, Poelmans, \& Blomert, 2005; Bonte, Poelmans, \& Blomert, 2007; Mitterer \& Blomert, 2003; Näätänen et al., 1997; Winkler et al., 1999) and language specific phonotactic regularities (Bonte et al., 2005; Bonte et al., 2007). These properties make the MMN the appropriate tool for investigating timing and degree of automaticity of letter influences on speech sound processing. In the Froyen et al. (2008) study the MMN evoked by a deviant speech sound /o/ violating a standard speech sound /a/ (auditory only experiment) was compared with the MMN evoked by the same deviant speech sound /o/, now not only violating the standard speech sound /a/, but also the simultaneously presented standard letter "a" (audiovisual experiment). The MMN amplitude evoked in the audiovisual experiment was enhanced in comparison with the MMN amplitude in the auditory only experiment, presumably caused by the double cross-modal violation. Given the early and automatic evocation of the MMN, this enhancement strongly points to early and automatic letter - speech sound integration in experienced adult readers.

To understand what the nature of letter - speech sound processing in dyslexia is, we must first know how this integration process develops in non-impaired readers. To this aim beginner and advanced but not yet experienced readers were investigated in a subsequent study, using the exact same MMN-design as described above (Froyen, Bonte, van Atteveldt, \& Blomert, 2009). The beginner readers in grade two had completed at least one year of reading experience and the advanced readers had completed at least four years of reading experience. In spite of the fact that beginner readers all showed full letter knowledge mastery, they did not show any indication of early and automatic letter - speech sound integration, i.e. no effect of letters on speech sound processing within the MMN time-window. Although highly experienced readers only showed automatic integration of letters and speech sounds if these were presented simultaneously (Froyen et al., 2008; Van Atteveldt, Formisano, Blomert et al., 2007), the advanced readers only revealed early and automatic integration if letters preceded speech sounds by 200 ms. Thus, even after four years of reading instruction in a relatively transparent orthography, letter - 
speech sound integration appeared not yet fully developed. Interestingly, both age groups also revealed a second systematic late effect (at $650 \mathrm{~ms}$ after stimulus onset) of letter - speech sound processing, which was not observed in the earlier adult study (Froyen et al., 2008). The results of both studies taken together indicate that these late effects only occurred if early integration was still developing. It was therefore suggested that the early effect of letters on speech sound processing reflected automatic integration, while the late effect was assumed to reflect a not yet automatic association process (Froyen et al., 2009). The development from mere association to automatic integration of letters and speech sounds seems to involve several years of reading experience even in non-impaired readers. These findings once again question the assumption of the establishment of stable letter - speech sound associations within months of reading instruction in non-impaired readers and the explanatory value of 'unstable' letter - speech sound relations in the context of the phonological deficit theory of dyslexia. The availability of a suitable method to measure, pre-attentively and with high temporal resolution, neural correlates of letter - speech sound processing, makes it possible to investigate the widely assumed, but rarely tested, 'unstable' letter - speech sound associations in dyslexic children.

\section{The present study}

We investigated the brain responses to letter - speech sound pairs in eleven year old dyslexic children (grade 5) with the exact same MMN paradigm as was used to study letter - speech sound processing in adult (Froyen et al., 2008), advanced (grade 5) and beginner (grade 1) non-impaired readers (Froyen et al., 2009). This allowed us to evaluate any signs of automatic letter - speech sound processing in the previously determined relevant early and late time-windows, by comparing the present findings with the previous findings for non-impaired reading chronological age matched peers as well as with those for the younger reading level matched readers. Since the time-window for integration had been shown to be critical in these studies we systematically manipulated the time-window of stimulus presentation: the letter appeared either $200 \mathrm{~ms}$ before speech sound onset, at $200 \mathrm{~ms}$ stimulus onset asynchrony (SOA), or the letter and speech sound were presented simultaneously, at 0 ms SOA.

We predict first a typical MMN in the auditory only condition since deviants and standards are clearly distinguishable from each other. Secondly, considering the indications for less successful integration of letters and speech sound in children with dyslexia (see above), we expected no modulation or a smaller enhancement of the MMN-amplitude in the audiovisual conditions in comparison with age matched controls. Thirdly, since dyslexic 11 year old readers show full letter knowledge mastery in addition to equal accuracy of letter - speech sound identification and dis- 
crimination in comparison with age matched non-impaired readers (Blomert \& Vaessen, 2009), we predicted a similar late association effect of letters on speech sound processing as observed previously in age matched non-impaired readers (Froyen et al., 2009).

\section{Methods}

Participants

Eighteen subjects participated in all three conditions. Data of two subjects were discarded due to excessive movement, retaining 16 subjects in the analysis (four female, range $10 ; 0$ to $12 ; 07$, mean age $11 ; 05$ years). All children were given a present for participation. Informed consent was obtained from the children and the parents. Approval for the study was granted by the Ethical Committee of the Faculty of Psychology at the University of Maastricht. All participants were native Dutch speakers. The participants were recruited from the Regional Institute of Dyslexia (RID), one of the major specialized dyslexia institutes in The Netherlands. The children in this group were monolingual native speakers of Dutch, diagnosed as dyslexic without any other dysfunctions ( $I Q \geq 85$, no hearing, neurological or psychological problems) after an extensive cognitive psycho-diagnostic procedure by the RID. We tested reading performance of the included participants in the same week as the ERP measurement by means of a combined word and pseudoword standardized test (Blomert \& Vaessen, 2009). Each and every participant performed within the lowest 10th percentile rank (group mean percentile rank = 5.0; SE 1.3).

\section{Stimuli}

Stimuli were speech sounds, /a/ (384 ms) and /o/ (348 ms), and the visually presented single letter "a". for comparison purposes we used the same stimuli as used in the adult study (Froyen et al., 2008) and in the developmental study (Froyen et al., 2008). To ensure a typical MMN reflecting speech sound processing, we used natural vowels (Näätänen, 2001). Furthermore, we choose the vowels /o/ and /a/, since these speech sounds were likely to evoke an $\mathrm{MMN}$ in dyslexic children. Csépe (2003) used consonant (/ba/ vs. /pa/ and /ba/ vs. /ga/) and vowel contrasts (/e/ vs /i/ and /e/ vs /o/) to investigate phonemic processing in dyslexia, and found that dyslexic children showed a typical MMN to vowels that, like the /a/ and /o/, differ in rounding.

Speech sounds were digitally recorded (sampling rate $44.1 \mathrm{kHz}, 16$ bit quantization) from a Dutch female speaker. Recordings were band-pass filtered $(180-10.000 \mathrm{~Hz})$, resampled at $22.05 \mathrm{kHz}$ and matched for loudness with Praat software (Boersma \& Weenink, 2002). The sounds were presented binaurally through loudspeakers at about $65 \mathrm{~dB}$ SPL. The letter was presented in white on a black background in the 
centre of a computer screen for $500 \mathrm{~ms}$, printed in lower case font "Arial" at font size 40, approximately $1.5 \mathrm{~cm}$ high and $1 \mathrm{~cm}$ wide. The distance from the screen to the subject was kept at $70 \mathrm{~cm}$. Consequently stimuli had a visual angle of approximately 1.2 by 0.8 degrees. During fixation periods, a white fixation cross was presented in the centre of the screen. The visually presented letter was always an "a", irrespective of whether the standard speech sound /a/ or the deviant speech sound /o/ was presented.

\section{Design and procedure}

In total, there were three different conditions, one auditory and two audiovisual conditions. In all three conditions subjects listened to the speech sounds /a/ (standard, 90\%) and /o/ (deviant, 10\%). In the auditory condition subjects listened to these speech sounds while watching a silent movie. In the two audiovisual conditions subjects listened to the same speech sounds, while watching single letters. Children were instructed not to move their eyes and sit quietly. To ensure that subjects were consistently focusing the screen, in the audiovisual conditions, periodically a full color picture of a present appeared in between trials. Children were instructed to press a button when this picture was presented. The two audiovisual conditions differ only with regard to the stimulus onset asynchrony (SOA) between the presentation of the letter and the speech sound. The letter appeared simultaneously (AV0) with the speech sound or 200 ms earlier (AV200). Each condition consisted of 4 experimental blocks with 534 trials each. Trial length was always 1250 $\mathrm{ms}$. The three conditions (A, AV0 and AV200) were presented in two sessions. In the first session one of the audiovisual conditions was presented, immediately followed by the auditory condition, while in the second session the remaining audiovisual condition was presented. The order of the audiovisual conditions (first or second session) was balanced over subjects.

\section{Recording and analysis}

EEG data were recorded in a sound-attenuating and electrically shielded room $(0.01$ - $50 \mathrm{~Hz}$, sampling rate $250 \mathrm{~Hz}$ ) from 30 electrode positions (Extended International 10-20 system, (Nuwer et al., 1998)) relative to a nose reference. Eye-movements and blinks were measured with bipolar VEOG/HEOG channels. All electrode impedance levels (EEG and EOG) were kept below $5 \mathrm{k} \Omega$. EEG data were epoched from -50 to $850 \mathrm{~ms}$ relative to stimulus onset, baseline corrected $(50 \mathrm{~ms}$ pre-stimulus interval), and $1-30 \mathrm{~Hz}$ band pass filtered (NeuroScan 4.2). Epochs containing data exceeding a maximum voltage criterion of $75 \mu \mathrm{V}$ were rejected. Standards immediately following deviants were not included in the analyses. The raw EEG data were corrected for vertical eye-movements (i.e. blink artifacts; (Semtlitsch, Anderer, Schuster, \& Presslich, 1986)). Subjects with over 30\% rejected epochs, i.e. 90 ep- 
ochs on a total of 300 per condition, were excluded from analysis. The resulting cleaned data sets did not show any confounds due to motion or eye-movement artifacts. Epochs were averaged separately for each condition and each participant. Difference waves were calculated by subtracting the ERPs to the standard condition from ERPs to the deviant condition.

Statistical analyses were performed on four electrodes covering the frontocentral ( Fz, Cz, FC3, FC4) regions of the brain to investigate the auditory MMN and the late negativity as reported in the developmental study (Froyen et al., 2009). From the difference waveforms we derived two dependent variables per time-window for each electrode: (I) the latency measured at the maximum amplitude within each predefined time-window and (II) the amplitude measured as the mean amplitude across 50 ms centered around the individual peak latency (separately for each subject, condition and electrode). We used the mean area amplitude rather than the peak amplitude as it is considered a more reliable measure. Consequently, in the present study, the term amplitude always refers to the mean area amplitude. All reported $p$-values from the repeated measures ANOVA were Greenhouse-Geisser corrected and all post-hoc tests were Bonferroni-corrected.

\section{Results}

First, the MMN to speech sounds in isolation and in the context of letters are presented. Secondly, we analyzed the data in search of a Late Negativity to speech sounds in isolation and a possible modulation in the context of letters. Furthermore, a correlation of the effect of letters on speech sound processing with reading measures was calculated. Finally, the results of the present study are compared with the results of the exact same experiment conducted with typically reading eight and eleven year olds (Froyen et al., 2009).

\section{Effect of letter on the MMN to speech sounds?}

Auditory only condition: We first analyzed the auditory condition to probe a typical $M M N$ evoked by a deviant speech sound in a standard speech sound context. Figure $1 \mathrm{~A}$ shows grand average ERPs for standard and deviant stimuli and difference waveforms (deviant minus standard) obtained in the auditory condition as measured at the central electrode sites ( $\mathrm{Fz}, \mathrm{Cz}, \mathrm{Pz}$ and $\mathrm{Oz}$ ). The speech sound deviant elicited an MMN with average peak latency of $164 \mathrm{~ms}$ (averaged over the four frontocentral electrodes, $30 \mathrm{~ms}$ SD) after the onset of the auditory stimulus (Table 1), with a topographical distribution (Figure 2) that is typically reported for the MMN (Picton, Alain, Otten, Ritter, \& Achim, 2000; Schröger, 1998). A one-sampled t-test of the mean area amplitudes of the difference waves at the frontocentral electrode sites (Fz, Cz, FC3 and FC4) revealed significant differences from zero at all four frontocentral electrode sites, indicating a typical $\mathrm{MMN}$ evoked by deviant speech sounds in 
children with dyslexia: at Fz, $t(15)=-5.15 ; p<0.001$, at $\mathrm{Cz}, t(15)=-5.27 ; p<0.001$, at FC3, $t(15)=-5.42 ; p<0.001$ and at FC4, $t(15)=-4.53 ; p<0.001$.

To assure that the dyslexic participants were able to discriminate the speech sounds in isolation equally well as non-impaired readers, we compared the MMN obtained in the present study with the MMN obtained in previous studies with non-impaired adults (Froyen et al., 2008) and children (Froyen et al., 2009). A repeated measures ANOVA on the mean area amplitudes was performed with electrode ( $F z, C z, F C 3$ and FC4) as within subject factor and group (dyslexic, beginner, advanced and experienced readers) as between subject factor. There was no interaction effect between electrode and group, $F(9,153)<1$ and no main effect of group, $F(3,51)<1$, indicating no speech sound discrimination problems in the early time-window of the MMN.

Audiovisual versus auditory only condition: To check for an effect of letters on early speech sound processing, the MMN evoked in the auditory condition was compared with the MMN in both audiovisual conditions (AVO and AV200). In all 3 conditions the deviant speech sound stimulus elicited a negativity with an average peak latency (averaged over the four frontocentral electrodes) at $170 \mathrm{~ms}$ (27 ms SD) after auditory stimulus onset (Figure 1 light grey rectangle), with a typical topographical distribution of the MMN (Figure 2). Average peak latencies (averaged over the four frontocentral electrodes) were $170 \mathrm{~ms}$ ( $23 \mathrm{~ms} \mathrm{SD}$ ) for AVO and $177 \mathrm{~ms}$ (27 ms SD) for AV200 after the onset of the auditory stimulus (Table 1). The latency values are reported with respect to the onset of the auditory stimulus. A repeated measures ANOVA on the latency of the MMN over the conditions did not show a significant effect on latency, $F(2,30)=1.4, p=0.261$.

Subsequently, amplitude properties of ERP activity in the MMN time-window were analyzed using a mixed ANOVA with two within-subject factors, electrode sites (4) and condition (3). There was no significant interaction between electrodes and condition, $F(6,90)=1.01, p=.409$, and no main effect of condition, $F(2,30)=1.63$; $p=0.219$. The similarity of the topographical distribution, latency and amplitude of the $\mathrm{MMN}$ in all three conditions indicated similar speech sound processing in this early time-window unaffected by letters in the audiovisual experiment. 
A. Auditory only

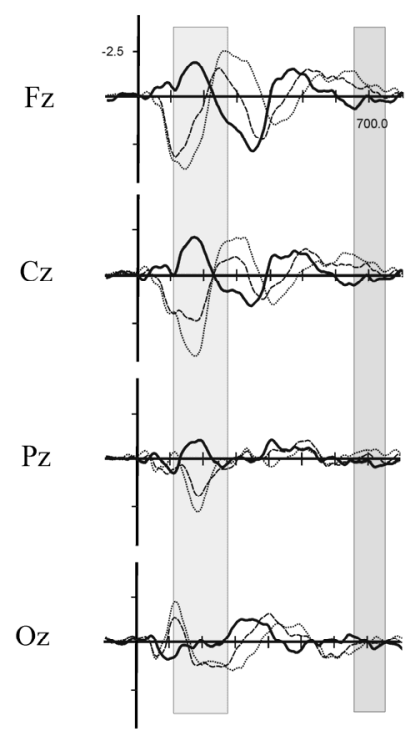

B. Audiovisual $0 \mathrm{~ms}$ SOA
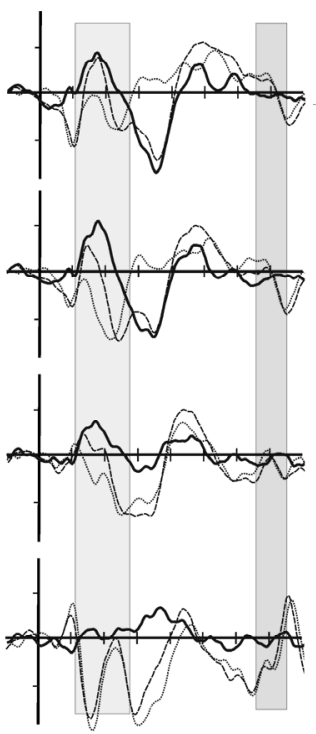

C. Audiovisual $200 \mathrm{~ms} \mathrm{SOA}$
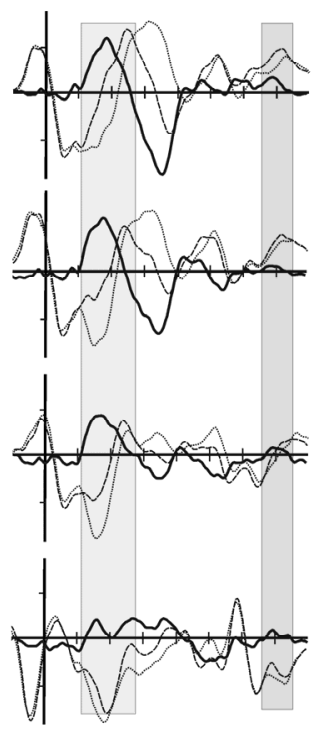

\begin{tabular}{lll} 
MMN time window & $\ldots . . . . . . .$. & Standard grand average wave \\
Late Negativity time window & --- & Deviant grand average wave \\
\hline & Difference wave
\end{tabular}

Figure 1. Grand average and difference waves as observed on four midline electrodes ( $F z, C z, P z$ and $O z)$ in the three conditions. The grey bars indicate the time-windows of interest.

\section{Effect of letter on the Late Negativity to speech sounds?}

Auditory only condition: Clearly, in the auditory condition, the dyslexic children did not evoke a Late Negativity to a deviant speech sound. Around $650 \mathrm{~ms}$ after stimulus onset deviant and standard grand-average waves reveal similar amplitudes resulting in a difference wave remaining around zero (Figure 1A, Figure 2 solid line). Consequently, one-sampled t-tests of the mean area amplitudes of the difference waves around $650 \mathrm{~ms}$ revealed no significant differences from zero at all four frontocentral electrode sites, at $\mathrm{Fz}, t(15)<1$, at $\mathrm{Cz}, t(15)<1$, at FC3, $t(15)<1$ and at FC4, $t(15)<1$.

Auditory versus audiovisual conditions: Within the group of children with dyslexia a repeated measures ANOVA (condition $x$ electrode) of the latency measures showed no interaction effect, $F(6,90)<1$, and no main effect of condition $F(2,30)<1$. A repeated measures ANOVA (condition $x$ electrode) of the amplitude measures, however, revealed no significant interaction effect, $F(6,90)<1$, but a significant main effect of condition, $F(2,30)=9.76 ; p=0.003$. Post hoc t-tests (Bonferroni corrected) revealed a significant difference between the auditory and the audiovisual 
condition AV200, $p=.004$, and a significant difference between the audiovisual condition AVO and the audiovisual condition AV200, $p<.001$, but not between the auditory condition and the audiovisual condition AV0, $p=1$. Apparently, at $650 \mathrm{~ms}$ after speech sound onset, there is a systematic influence of letters on speech sound processing in dyslexic readers, but only when the letter is presented $200 \mathrm{~ms}$ before the speech sound.

Table 1. Peak latency and mean area amplitude measures on the four frontocentral electrodes with standard errors of the mean of dyslexic readers for the three conditions

\begin{tabular}{|c|c|c|c|}
\hline Difference waves & Auditory & Audiovisual $0 \mathrm{~ms}$ SOA & Audiovisual $200 \mathrm{~ms}$ SOA \\
\hline \multicolumn{4}{|l|}{ MMN } \\
\hline \multicolumn{4}{|c|}{ Peak latency \pm SE (ms) } \\
\hline $\mathrm{Fz}$ & $153 \pm 9$ & $169 \pm 6$ & $181 \pm 7$ \\
\hline $\mathrm{Cz}$ & $167 \pm 7$ & $169 \pm 6$ & $172 \pm 6$ \\
\hline FC3 & $165 \pm 7$ & $170 \pm 5$ & $176 \pm 6$ \\
\hline FC4 & $169 \pm 7$ & $170 \pm 6$ & $175 \pm 8$ \\
\hline \multicolumn{4}{|c|}{ Mean area \pm SE $(\mu \mathrm{V})$} \\
\hline $\mathrm{Fz}$ & $-2.14 \pm 0.4$ & $-2.21 \pm 0.4$ & $-3.06 \pm 0.3$ \\
\hline $\mathrm{Cz}$ & $-2.33 \pm 0.4$ & $-2.64 \pm 0.5$ & $-3.00 \pm 0.3$ \\
\hline FC3 & $-2.00 \pm 0.3$ & $-2.15 \pm 0.4$ & $-2.65 \pm 0.4$ \\
\hline FC4 & $-1.98 \pm 0.4$ & $-2.48 \pm 0.4$ & $-2.90 \pm 0.3$ \\
\hline \multicolumn{4}{|l|}{ IMMN } \\
\hline \multicolumn{4}{|c|}{ Peak latency \pm SE (ms) } \\
\hline $\mathrm{Fz}$ & $691 \pm 7$ & $678 \pm 9$ & $687 \pm 10$ \\
\hline $\mathrm{Cz}$ & $686 \pm 7$ & $678 \pm 9$ & $686 \pm 10$ \\
\hline FC3 & $683 \pm 9$ & $677 \pm 10$ & $691 \pm 9$ \\
\hline FC4 & $690 \pm 7$ & $678 \pm 8$ & $687 \pm 9$ \\
\hline \multicolumn{4}{|c|}{ Mean area $\pm \mathrm{SE}(\mu \mathrm{V})$} \\
\hline $\mathrm{Fz}$ & $0.09 \pm 0.3$ & $0.05 \pm 0.2$ & $-1.27 \pm 0.2$ \\
\hline $\mathrm{Cz}$ & $0.16 \pm 0.5$ & $0.15 \pm 0.2$ & $-1.16 \pm 0.3$ \\
\hline FC3 & $0.08 \pm 0.4$ & $0.11 \pm 0.3$ & $-1.18 \pm 0.2$ \\
\hline FC4 & $0.10 \pm 0.4$ & $-0.09 \pm 0.2$ & $-1.25 \pm 0.2$ \\
\hline
\end{tabular}

Correlations with behavioral measures: A single measure of the influence of the letter on speech sound processing around $650 \mathrm{~ms}$ was calculated in order to be able to check for correlations with behavioral measures. To obtain a single Late Negativity effect measure, the amplitude of the Late Negativity in the auditory only condition was subtracted from the amplitude in the audiovisual condition with $200 \mathrm{~ms}$ SOA. Correlations were calculated between this single Late Negativity effect measure and performance on word and pseudo word reading, phonological awareness and rapid naming. Since the reading test used for subject selection combined word and pseudoword reading (Blomert \& Vaessen, 2009), we now used the standardized scores on a one minute word reading (Brus \& Voeten, 1973) and a two minutes pseudoword reading task (Van den Bos, lutje Spelberg, Scheepsma, deVries, 1999) also administered before the electrophysiological measurement. Since accuracy of 
performance on phonological awareness tasks often reaches ceiling within the first years of reading instruction in transparent languages, we used the reaction times of the responses on the phoneme deletion task. We furthermore included speed scores for rapidly naming letters and digits.

x

Figure 2: Difference waves from the auditory and audiovisual experiments with the time windows of interest indicated with grey bars.

Only word reading correlated strongly with the Late Negativity effect measure, $r=$ $.632 ; p=.011$; pseudoword reading somewhat less $r=-.495 ; p=.060$ (see Figure 3). There was no significant correlation with scores on rapid naming, $r=.258 ; p=.354$, or phoneme deletion, $r=-.061 ; p=.829$. The negative correlations mean that a stronger effect of letters on the Late Negativity goes with better behavioral performance. A hierarchical regression analysis with word and non-word reading as predictor explained $42 \%$ of the variability in the Late Negativity effect measure (word reading alone explained already $40 \%$ of the variance). 

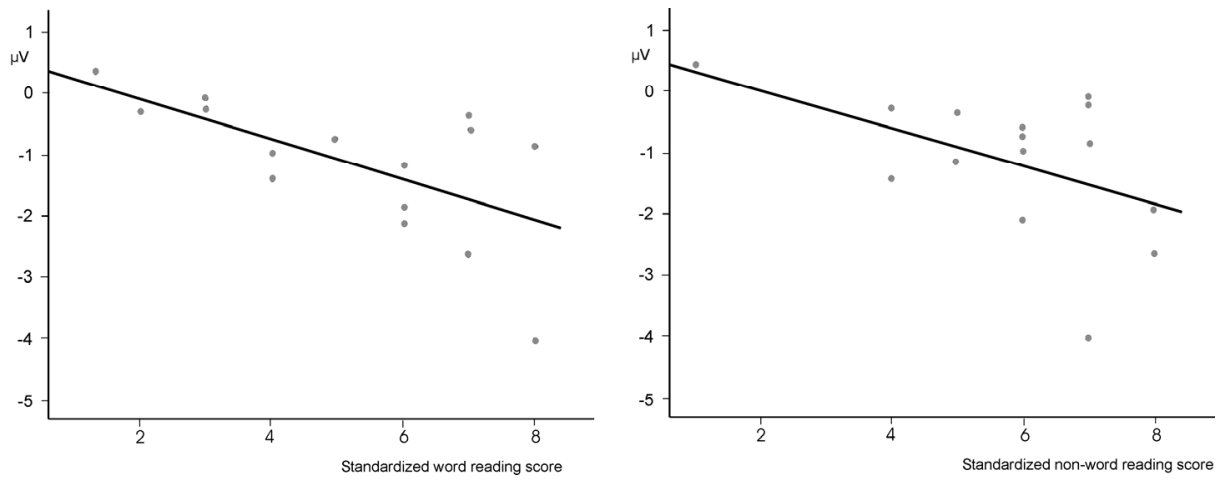

Figure 3: Correlation between word (left) and pseudoword (right) reading (standard scores; mean 10, SD 2) and the late negativity effect calculated per participant.

\section{Comparison with previously collected data:}

As summarized in table 2, the present results clearly resembled the previously found letter - speech sound processing pattern of beginner readers (Froyen et al., 2009), but differed in all aspects from the age matched advanced reading peers: In the early MMN time-window, letters had no effect on speech sound processing, while in the Late Negativity time-window, there was an effect of letters but only at $200 \mathrm{~ms}$ SOA. So, no letter - speech sound integration and no association if letters and speech sound occur in synchrony. Although fifth grade dyslexic children thus resemble non-impaired reading second graders in their processing of letter - speech sound associations, they clearly differ from them when processing speech sounds in isolation. Only beginner readers and not dyslexic children (this study), nor advanced (Froyen et al., 2009) or adult readers (Froyen et al., 2008), revealed any late negativities in response to deviant speech sounds alone. A repeated measures ANOVA on the amplitude of the Late Negativity in the auditory condition with electrode as a within subject factor and group as a between subject factor revealed no interaction, $F(9,150)<1$, but a significant main effect of group, $F(3,50)=4.19 ; p=0.010$. Post hoc t-tests (Bonferroni corrected) reveal no difference between the children with dyslexia and the advanced readers, $p=1$, or with the adult readers, $p=1$, but a significant difference with the beginner readers, $p=0.009$. Dyslexic readers thus revealed a normal pattern of speech sound processing adequate for their age, whereas beginner readers revealed less automaticity of speech sound discrimination. In summary (see Table 2), dyslexic readers distinguish between the presently used speech sounds in a similar way as peers and adults, but do not develop the automatic integration of letters and speech sounds characteristic of these same readers and presumably necessary for the development of fluent reading. 
Table 2. Comparison of dyslexic eleven year old children (present study) with non-impaired reading eight and eleven year old (Froyen et al., 2009) and adult readers (Froyen et al., 2008).

\begin{tabular}{|c|c|c|c|c|c|c|}
\hline & \multirow{2}{*}{$\begin{array}{l}\text { Unimodal } \\
\text { MMN }\end{array}$} & \multicolumn{2}{|c|}{ Crossmodal effect } & \multirow{2}{*}{$\begin{array}{l}\text { Unimodal } \\
\text { Late Neg }\end{array}$} & \multicolumn{2}{|c|}{ Crossmodal effect } \\
\hline & & SOA 0 & SOA 200 & & SOA 0 & SOA 200 \\
\hline \multicolumn{7}{|l|}{ Present study } \\
\hline 11 y dyslexic: & $\underline{\text { YES }}$ & NO & NO & NO & NO & $\underline{\text { YES }}$ \\
\hline \multicolumn{7}{|l|}{ Froyen et al. 2008} \\
\hline 7 y non-impaired: & $\underline{\text { YES }}$ & NO & NO & $\underline{\text { YES }}$ & NO & $\underline{\text { YES }}$ \\
\hline 11 y non-impaired: & $\underline{\text { YES }}$ & NO & $\underline{\text { YES }}$ & NO & $\underline{\text { YES }}$ & NO \\
\hline \multicolumn{7}{|l|}{ Froyen et al. 2009} \\
\hline Adult non-impaired: & YES & YES & NO & NO & NO & NO \\
\hline
\end{tabular}

\section{Discussion}

The present study explored the neural correlates of letter - speech sound processing in dyslexic children. More particularly we wanted to know if dyslexic children revealed anomalies during the processing of letter - speech sound pairs and if so what the nature of this anomaly would be. To this aim we used an MMN paradigm in which we presented speech sounds in isolation and in the context of corresponding letters. We hypothesized that the deviant speech sound would elicit a typical MMN in both conditions, whereby amplitude changes of the MMN in the cross-modal condition were hypothesized to signal early and automatic letter - speech sound integration as revealed in earlier studies with non-impaired readers (Froyen et al., 2008). Since a previous study also revealed systematic letter - speech sound effects in eight and eleven year old children at a much later time -window (Froyen et al., 2009) we focused on the occurrence of possible early automatic integration effects and late association effects of letters and speech sounds in dyslexic children with four years of reading instruction. The overall results revealed that, in contrast to chronological age matched non-impaired readers, dyslexic children did not automatically integrate letters and speech sounds even after four years of reading experience. At the same time, they did show normal brain responses to the same speech sounds if presented without letters.

\section{$M M N$}

Before checking for early and automatic cross-modal effects of letters on speech sound processing, it is important to assure a typical MMN in dyslexic readers. Dyslexic readers elicited a typical $M M N$ in terms of amplitude, latency and topographical distribution (Picton et al., 2000; Schröger, 1998). We therefore compared the MMN to speech sounds in isolation obtained in dyslexics with the MMN obtained in beginner, advanced (Froyen et al., 2008) and adult readers (Froyen et al., 2008). No differences were found in amplitude, latency, or topographical distribution with the 
MMN of the non-impaired readers from the previous studies. In agreement with an earlier study using vowels differing in 'rounding' (Csépe, 2003), the results indicated that dyslexic readers discriminated the present speech sounds equally well as nonimpaired readers.

Next, the influence of letters on speech sound processing in dyslexic readers was investigated. Comparing the MMN obtained in the auditory condition with the MMN obtained in the two audiovisual conditions (AVO and AV200) revealed no differences in amplitude, latency, or topographical distribution. Apparently, letters did not influence early speech sound processing. Consequently, there is no indication for early and automatic letter - speech sound integration in dyslexic children, despite four years of reading experience. This sharply contrasts with the results from non-impaired age matched readers (Froyen et al., 2008), who did show early and automatic integration effects. In fact, the present results resembled the results from the beginner readers with approximately one year of reading experience. This means that eleven year old children with dyslexia, like eight year old non-impaired readers, do not automatically integrate letters with speech sounds.

\section{Late Negativity}

Because of the lack of early integration effects, we hypothesized that dyslexic children would elicit a late letter - speech sound association effect reflecting their ability to accurately link letters with their corresponding speech sounds as shown in previous behavioral studies (e.g., Blomert \& Vaessen, 2009). This was indeed the case: Dyslexic readers did show a late association effect. However in the previous developmental study, age matched non-impaired readers only revealed such an association effect, if the stimuli were presented in synchrony, that is, in the very narrow time-window that was also characteristic for adult early cross-modal integration effects (Froyen et al., 2008; Van Atteveldt, Formisano, Blomert et al., 2007). Since the present dyslexic readers did not show any late effects in this narrow timewindow, but only revealed late association effects in a much wider time-window, we interpret this as an indication that even the association between corresponding letters and speech sounds is still weak after four years of reading instruction. In fact, the results of the dyslexic children mimic the results reported for beginner readers who experienced only one year of reading instruction (Froyen et al., 2008). Although previous behavioral data reported no accuracy differences in letter - speech sound congruency detection between dyslexic and non-impaired readers anymore in grade five, dyslexic children still were significantly slower than their non-impaired reading peers (Blomert \& Vaessen, 2009). The present results suggest that these longer response latencies are a consequence of a weaker neural association between corresponding letters and speech sounds. 
The present results confirm the existence of a Late Negativity around $650 \mathrm{~ms}$ after stimulus onset, first described in the developmental study by Froyen and coautors (2008). This component is clearly distinguishable from the earlier described Late MMN appearing around 450 ms (Cheour, Korpilahti, Martynova, \& Lang, 2001; Korpilahti, Krause, Holopainen, \& Lang, 2001). Except in beginner readers (Froyen et al., 2008), the Late Negativity was not evoked by speech sounds alone and, consequently, might reflect cross-modal association effects. It is unlikely that the Late Negativity is specifically reflecting letter - speech sound association and whether or not this component reflects non-specific audiovisual association or even more general association processes remains to be investigated. However, the correlations of this late negativity particularly with word reading performance warrant further explorations of the specificity of the processes involved. Although there is an obvious trade off with the total duration time of an experiment, the present study shows that it might be worthwhile to consider increasing epoch lengths whenever one conjectures cross-modal association effects.

In closing we would like to note that in our previous developmental study we could only speculate about the contributions of brain maturation and increasing reading experience to the observed ERP effects (Froyen et al., 2008). The present results allow us to infer that it was likely reading experience and level of reading expertise and not brain maturation per se which constituted the main source of the previously observed neural activation differences between eight and eleven year old readers. If brain maturation had been the main contributor, dyslexic eleven year old children would probably have resembled the results of the age matched nonimpaired readers much more closely.

\section{Theoretical Implications}

Mainstream theory of dyslexia assumes that reading problems encountered by dyslexic children are a consequence of a phonological deficit (Vellutino et al., 2004; Ziegler \& Goswami, 2005). These phonological problems are assumed to cause reading failure, because, when learning to read, a dyslexic child is taught to associate these ill-formed phonological representations to letters, resulting in 'unstable' letter - speech sound pairs (see introduction). After more than a quarter of a century of research into the assumed causal phonological deficits in dyslexia, a clear description of the mechanisms involved in letter - speech sound processing, and the failure thereof because of phonological problems, is still lacking. The present results confirm that dyslexics do exhibit problems when associating letters and speech sounds, albeit in a different form than previously assumed and, interestingly, in the context of intact processing of the same speech sounds if processed in isolation.

Stable letter - speech sound associations are assumed to be required for learning to read (Ehri, 2005; Frith, 1985). The present results build on this assumption by show- 
ing that it is necessary to refine the meaning of the concept of "letter - speech sound association" to understand the way it may impact reading. The present results strongly indicated that knowledge of the relations between letters and speech sounds is not sufficient for adequate reading development; i.e., declarative knowledge of letter - speech sound associations is not the same as the procedural use of this knowledge during reading. In stead, learning to read fluently requires that learned associations between letters and speech sounds develop into an automatically integrated letter - speech sound compound. Dyslexic children not only seemed to fail at integrating letter - speech sound pairs, but even the mere associations between letters and speech sounds were still very weak after four years of reading instruction. Support for these results comes from a recent neuro-imaging study showing that even adult dyslexics with at least 10 years of reading instruction and years of compensatory reading remediation did exhibit anomalous letter - speech sound processing (Blau et al., 2009). Adult dyslexic readers did show comparable brain activity as their non-impaired reading peers, when processing congruent letter - speech sound pairs. However, they also processed incongruent letter - speech sound pairs as if they were actually congruent. In contrast, non-impaired readers immediately suppressed these incongruent letter speech sound pairs. The discrepancy between this evidence for an almost life-long failure to adequately integrate a few dozen letter- speech sound pairs (in transparent languages) stands in bewildering contrast with, first, the widespread assumption that children learn letter speech sound associations within a few months (e.g. Ziegler \& Goswami, 2005) and, second, the observation that dyslexic and non-impaired readers all show ceiling performance on letter knowledge tasks at the end of first grade (Aarnoutse et al., 2000; Willems et al., submitted). We interpreted the longer response latencies of the dyslexics for letter - speech sound discrimination reported in Blomert and Vaessen (2009) as a consequence of the weak association and absence of integration of letters and speech sounds found in the present study. We would also like to advance this interpretation as a tentative explanation for the observation that reading fluency is the best defining and hardest to remediate aspect of dyslexic reading in transparent orthographies (Mann \& Wimmer, 2002).

This leaves us with the notorious question of the chicken and the egg regarding the causes and consequences of reading deficits in dyslexia. The phonological deficit hypothesis assumes that degraded phonological representations hamper the acquisition of stable letter - speech sound associations resulting in deficient reading and spelling development (Vellutino et al., 2004; Ziegler \& Goswami, 2005). However, our dyslexic children did not differ from their age matched controls in the perception of speech sounds in isolation. The MMN to speech sounds in isolation in the present study did not differ from the MMN in beginner, advanced (Froyen et al., 2009) and adult non-impaired readers (Froyen et al., 2008), indicating sufficiently effective phoneme categories. The present finding that the same speech sounds, 
which were correctly discriminated, were only weakly associated with their corresponding letters, therefore does not seem to support the mainstream line of reasoning from a phonological deficit to letter - speech sound problems, but seems to point to a generic letter - speech sound association problem. This interpretation is supported by the finding that the poor phonological awareness performance did not correlate with the late negativity effect (presumably an index for the quality of letter - speech sound association), but reading performance clearly did. In sum, we think that the present findings point to a generic letter - speech sound association problem in dyslexia, which does not seem to be caused by a phonological problem.

The causal role of a phonological deficit in dyslexic reading problems has been questioned from its inception on different and equally valid grounds (Castles \& Coltheart, 2004; Mann \& Wimmer, 2002; Morais, Bertelson, Cary, \& Alegria, 1986; Morais, Cary, Alegria, \& Bertelson, 1979; Perfetti, Beck, Bell, \& Hughes, 1987; Wagner, 1987; Wimmer \& Hummer, 1990). If the phonological deficit is indeed "more consequence than precondition" (Wimmer \& Hummer, 1990) of dyslexic reading problems, than it is also unlikely that they are the cause of 'unstable' letter - speech sound associations. We therefore advance the possibility that the severe letter speech sound association problems revealed in this study may actually constitute one of the generic and proximal causes of dyslexic reading problems. This has the evident advantage that such a specific cross-modal association deficit has a direct and theoretically and empirically valid relation with learning to read, a relation much less obvious in case of a causal phonological deficit.

In sum, fifth grade dyslexic children revealed no signs of automatic letter - speech sound integration, despite four years of reading instruction and accurate letter knowledge. In stead they did show weak letter - speech sound associations, comparable to beginner readers with only one year of reading instruction. Interestingly they did not exhibit problems in processing the same speech sounds without letters. We interpreted these findings as the manifestation of a generic cross-modal deficit in letter - speech sound associations, very likely one of the proximal causes of reading problems in dyslexia.

\section{References}

Aarnoutse, C., van Leeuwe, J., \& Verhoeven, L. (2000). Ontwikkeling van beginnende geletterdheid (development of beginning literacy). Pedagogische Studiën, 77, 307 - 325.

Alho, K. (1995). Cerebral Generators of mismatch negativity (MMN) and its magnetic counterpart (MMNm) elicited by sound changes. Ear \& Hearing, 16, 38-51.

Blau, V., Atteveldt, N., Ekkebus, M., Goebel, R., \& Blomert, L. (2009). Reduced neural integration of letters and speech sounds links phonological and reading deficits in adult dyslexia. Current Biology, 19,503 - 508.

Blau, V., van Atteveldt, N., Formisano, E., Goebel, R., \& Blomert, L. (2008). Task-irrelevant visual letters interact with the processing of speech sounds in heteromodal and unimodal cortex. European journal of neuroscience, $28,500-509$. 
Blomert, L., \& Vaessen, A. (2009). 3DM Differential diagnostics for dyslexia: Cognitive analysis of reading and spelling. Amsterdam: Boom Test Publishers.

Boersma, P., \& Weenink, D. (2002). Praat 4.0: a system for doing phonetics with the computer. Amsterdam: Universiteit van Amsterdam.

Bonte, M., Mitterer, H., Zellagui, N., Poelmans, H., \& Blomert, L. (2005). Auditory cortical tuning to statistical regularities in phonology. Clinical Neurophysiology, 116, 2765-2774.

Bonte, M., Poelmans, H., \& Blomert, L. (2007). Deviant neurophysiological responses to phonological regularities in speech in dyslexic children. Neuropsychologia, 45, 1427-1437.

Brus, B. T., \& Voeten, M. J. M. (1973). Een-minuuttest, vorm A en B. Nijmegen: Berkhout.

Calfee, R. C., Venezky, R. L., \& Chapman, R. S. (1969). Pronounciation of synthetic words with predictable and unpredictable sound correspondences. Madison: University of Wisconsin, Research and Development Center.

Castles, A., \& Coltheart, M. (2004). Is there a causal link from phonological awareness to succes in learning to read? Cognition, 91, 77 - 111.

Cheour, M., Korpilahti, P., Martynova, O., \& Lang, A. H. (2001). Mismatch negativity and late discriminative negativity in investigating speech perception and learning in children and infants. Audiology and Neuro-otology, 6, 2-11.

Csépe, V. (2003). Dyslexia: different Brain, Different Behavior. New York: Kluwer Academic/ Plenum Publishers.

Ehri, L. C. (2005). Development of sight word reading: phases and findings. In M. J. Snowling \& C. Hulme (Eds.), The science of reading: A handbook (pp. 135 - 145). Oxford: Blackwell Publishing.

Fox, E. (1994). Grapheme-phoneme correspondence in dyslexic and matched control readers. British Journal of Psychology, 85, 41-53.

Frith, U. (1985). Beneath the surface of developmental dyslexia. In K. E. Patterson, J. C. Marshall \& M. Coltheart (Eds.), Surface dyslexia. London: Routledge \& Kegan-Paul.

Froyen, D., Bonte, M., van Atteveldt, N., \& Blomert, L. (2009). The long road to automation: Neurocognitive development of letter-speech sound processing. Journal of Cognitive Neuroscience, 21, 567 580.

Froyen, D., van Atteveldt, N., Bonte, M., \& Blomert, L. (2008). Cross-modal enhancement of the MMN to speech sounds indicates early and automatic integration of letters and speech sounds. Neuroscience Letters, 430, 23-28.

Giard, M. H., Perrin, F, Pernier J, Bouchet P. (1990). Brain generators implicated in the processing of auditory stimulus deviance: a topographic event-related potential study. Psychophysiology, 27, 627640.

Hardy, M. H., Smythe, P. C., Stennet, R. G., \& Wilson, H. R. (1972). Developmental patterns in elemental reading skills: phoneme-grapheme and grapheme-phoneme correspondences. Journal of Educational Psychology, 63, 433-436.

Hashimoto, R., \& Sakai, K. L. (2004). Learning letters in adulthood: direct visualization of cortical plasticity for forming a new link between orthography and phonology. Neuron, 42, 311-322.

Korpilahti, P., Krause, C. M., Holopainen, I., \& Lang, A. H. (2001). Early and late mismatch negativity elicited by words and speech-like stimuli in children. Brain and Language, 76, 332-339.

Lyon, G. R., Shaywitz, S. E., \& Shaywitz, B. A. (2003). Towards a definition of dyslexia. Annals of Dyslexia, 53, 1-14.

Mann, V., \& Wimmer, G. (2002). Phoneme awareness and pathways into literacy: A comparison of German and American children. Reading and Writing, 15, 653-682.

Mitterer, H., \& Blomert, L. (2003). Coping with phonological assimilation in speech perception: evidence for early compensation. Percept Psychophys, 65, 956-969.

Morais, J., Bertelson, P., Cary, L., \& Alegria, J. (1986). Literacy training and speech segmentation. cognition, 24, 45 - 64.

Morais, J., Cary, L., Alegria, J., \& Bertelson, P. (1979). Does awareness of speech as a saquence of phones arise spontaneously? Cognition, 49, 957 - 958. 
Näätänen, R. (1995). The Mismatch Negativity: A powerful tool for cognitive neuroscience. Ear and Hearing, 16, 6-18.

Näätänen, R. (2001). The perception of speech sounds by the human brain as reflected by the mismatch negativity (MMN) and its magnetic equivalent (MMNm). Psychophysiology, 38, 1-21.

Näätänen, R., Lehtokoski, A., Lennes, M., Cheour, M., Huotilainen, M., livonen, A., et al. (1997). Language-specific phoneme representations revealed by electric and magnetic brain responses. Nature, 385, 432-434.

Nuwer, M., Comi, G., Emerson, R., Fuglsang-Frederiksen, A., Guérit, J., Hinrichs, H., et al. (1998). IFCN standards for digital recording of clinical EEG. Electroencephalogr Clin Neurophysiol, 106, 259-261.

Perfetti, C. A., Beck, I., Bell, L., \& Hughes, C. (1987). Phonemic knowledge and learning to read are reciprocal: A longitudinal study of first grade children. Merrill-Palmer Quarterly, 33, 283-319.

Picton, T. W., Alain, C., Otten, L., Ritter, W., \& Achim, A. (2000). Mismatch negativity: different water in the same river. Audiol Neurootol, 5, 111-139.

Raij, T., Uutela, K., \& Hari, R. (2000). Audiovisual integration of letters in the human brain. Neuron, 28, 617-625.

Schröger, E. (1998). Measurement and interpretation of the mismatch negativity. Behavior Research Methods Instruments \& Computers, 30, 131-145.

Semtlitsch, H., Anderer, P., Schuster, P., \& Presslich, O. (1986). A solution for reliable and valid reduction of ocular artefacts, applied to the P300 ERP. Psychophysiology, 23, 695-703.

Share, D. L. (2008). On the anglocentricities of current reading research and practice: The perils of overreliance on an "outlier" orthography. Psychological bulletin, 134, 584 - 615.

Siegel, L. S., \& Faux, D. (1989). Aquisition of certain grapheme-phoneme correspondences in normally achieving and diabled readers. Reading and Writing, an interdisciplinary journal, 1, 37-52.

Snowling, M. J. (1980). The development of grapheme-phoneme correspondence in normal and dyslexic readers. Journal of Experimental Child Psychology, 29, 294-305.

Van Atteveldt, N., Formisano, E., Blomert, L., \& Goebel, R. (2007). The effect of temporal asynchrony on the multisensory integration of letters and speech sounds. Cerebral Cortex, 13, 962-974.

Van Atteveldt, N., Formisano, E., Goebel, R., \& Blomert, L. (2004). Integration of letters and speech sounds in the human brain. Neuron, 43, 271-282.

Van Atteveldt, N., Formisano, E., Goebel, R., \& Blomert, L. (2007). Top-down task effects overrule automatic multisensory responses to letter-sound pairs in auditory association cortex. Neurolmage, 36, 1345-1360.

Vellutino, F. R., Fletcher, J. M., Snowling, M. J., \& Scanlon, D. M. (2004). Specific reading disability (dyslexia): what have we learned in the past four decades? J Child Psychol Psychiatry, 45, 2-40.

Wagner, R. K., Torgesen, J.K. (1987). The nature of phonological processing and its causal role in the acquisition of reading skills. Psychological Bulletin, 101, 192-212.

Willems, G., Poelmans, H., Richardson, U., \& Blomert, L. (submitted). What preschool intervention in children at familial risk of dyslexia reveals about their core deficits and the way to treat them.

Wimmer, H., \& Hummer, P. (1990). How German-speaking first graders read and spell: Doubts on the importance of the logographic stage. Applied Psycholinguistics, 11, 349 - 368.

Winkler, I., Kujala, A., Tiitinen, H., Sivonen, P., Alku, P., Lehtokoski, A., et al. (1999). Brain responses reveal the learning of foreign language phonemes. psychophysiology, 36, 638-642.

Ziegler, J. C., \& Goswami, U. (2005). Reading acquisition, developmental dyslexia, and skilled reading across languages: a psycholinguistic grain size theory. Psychol Bull, 131, 3-29. 

Chapter 5

\section{Exploring the role of low level visual processing in letter - speech sound integration: a visual MMN study}

Froyen, D., Van Atteveldt, N., \& Blomert, L. (submitted). Exploring the role of low level visual processing in letter - speech sound integration: a visual MMN study 


\begin{abstract}
Letter - speech sound pairs can be seen as audiovisual objects. However, in contrast with for example audiovisual speech, the relation between visual and auditory properties of letters and speech sounds is artificial and learned only by explicit instruction. Nevertheless, in fluent readers, neurofunctional evidence indicates robust and automatic integration of letter - speech sound pairs. The arbitrariness of the audiovisual link together with the widespread usage of letter - speech sound pairs in alphabetic languages makes those audiovisual objects a unique subject for crossmodal research. Brain imaging evidence has indicated that heteromodal areas in superior temporal, as well as modality-specific auditory cortex are involved in letter - speech sound processing. The role of low level visual areas, however, remains unclear.

In this study the visual counterpart of the auditory mismatch negativity (MMN) is used to investigate the influences of speech sounds on letter processing. Letter and non-letter deviants were infrequently presented in a train of standard letters, either in isolation or simultaneously with speech sounds. Although previous findings showed that letters systematically modulate speech sound processing (reflected by auditory MMN amplitude modulation), the reverse does not seem to hold: our results did not show evidence for an automatic influence on letter processing by speech sounds (no visual MMN amplitude modulation). This apparent asymmetric recruitment of low level sensory cortices during letter - speech sound processing, contrasts with the symmetric involvement of these cortices in audiovisual speech, and is possibly due to the nature of the arbitrarily defined link between letters and speech sounds.
\end{abstract}




\section{Introduction}

The ability to rapidly integrate cross-modal sensations originating from a single object allows more efficient and profound processing of our environment. The neural network of audiovisual speech processing, a classic example of audiovisual integration, involves multisensory integration sites as well as low level auditory and visual sensory systems, presumably via feedback projections (Calvert et al., 1999; Calvert, Campbell, \& Brammer, 2000; Macaluso, George, Dolan, Spence, \& Driver, 2004). While recent studies revealed that multisensory as well as low level auditory processing are involved in a basic literacy skill such as letter - speech sound integration (Blau, van Atteveldt, Formisano, Goebel, \& Blomert, 2008; Hashimoto \& Sakai, 2004; Van Atteveldt, Formisano, Blomert, \& Goebel, 2007; Van Atteveldt, Formisano, Goebel, \& Blomert, 2004), the role of low level visual processing is less consistently reported and is the objective of the present study.

In alphabetic scripts, learning the associations between letters and speech sounds is a crucial step in reading acquisition (Ehri, 2005). Failure of this step during learning to read is proposed as a cause for reading problems in developmental dyslexia (Blau, Atteveldt, Ekkebus, Goebel, \& Blomert, 2009; McCandliss \& Noble, 2003; Vellutino, Fletcher, Snowling, \& Scanlon, 2004). Insights in the neurocognitive nature of letter - speech sound processing might therefore contribute to theories of normal and abnormal literacy development. A behavioral study, in which the nature of letter - speech sound processing was investigated, revealed differential influences of congruent and incongruent letter primes on reaction times in a speech sound identification task, suggesting automatic letter - speech sound integration (Dijkstra, Schreuder, \& Frauenfelder, 1989). Converging evidence from several recent neuro-imaging studies points to heteromodal areas in the Superior Temporal Sulcus (STS) as a crucial locus of this integration process (Blau, van Atteveldt, Formisano, Goebel, \& Blomert, 2008; Hashimoto \& Sakai, 2004; Raij, Uutela, \& Hari, 2000; Van Atteveldt, Formisano, Blomert, \& Goebel, 2007; Van Atteveldt, Formisano, Goebel, \& Blomert, 2004). In these studies, letter - speech sound pairs activated areas in STS stronger in comparison with single letters or speech sounds. Van Atteveldt and colleagues (2004; 2007a) furthermore showed that not only heteromodal areas in STS, but also early auditory areas (Heschl's sulcus and Planum Temporale) are involved in letter - speech sound integration. Congruent letter - speech sound pairs activated early auditory areas more in comparison with incongruent pairs (congruency effect). Based on these results the following neural mechanism for letter - speech sound processing was proposed: Sensory specific input in early sensory areas and letter - speech sound integration in STS, followed by feedback to "sensory specific" auditory areas modulating speech sound processing depending on the congruency of the letter - speech sound combinations (Blau, van Atteveldt, Formisano, Goebel, \& Blomert, 2008; Van Atteveldt, Formisano, Blomert, \& Goebel, 2007; Van Atteveldt, Formisano, Goebel, \& Blomert, 2004). 
In contrast with what is observed during audiovisual speech processing, no integration effect was found in low level visual areas during passive letter - speech sound processing (Van Atteveldt, Formisano, Blomert, \& Goebel, 2007; Van Atteveldt, Formisano, Goebel, \& Blomert, 2004). This indicates an asymmetry in the involvement of early sensory areas when integrating letters and speech sounds, which is in agreement with the asymmetry in the representations of letter - speech sound associations found on a behavioral level (Dijkstra, Frauenfelder, \& Schreuder, 1993; Hardy, Smythe, Stennet, \& Wilson, 1972). However, the involvement of low level visual areas has been reported in two studies (Blau, van Atteveldt, Formisano, Goebel, \& Blomert, 2008; Herdman et al., 2006). In a magnetoencephalography (MEG) study, subjects were required to make a congruency decision on the presented letters and speech sounds (Herdman et al., 2006). Cortical oscillations revealed congruency effects in low level visual areas between 250 and 500 ms. Such a congruency effect was not found for the same task when functional magnetic resonance imaging (fMRI) was used (Van Atteveldt, Formisano, Goebel, \& Blomert, 2007), suggesting that the use of a high temporal resolution method may be critical for revealing relevant effects. This suggestion seems contradicted by an fMRI-study by Blau and colleagues (2008), in which congruency effects were observed in extrastriate areas while subjects were identifying speech sounds co-occurring with congruent or incongruent letters. However, in this study, degraded letters were presented, possibly requiring stronger involvement of low level visual areas already during unimodal processing in comparison with non-degraded letters.

The role of low level visual areas during passive letter - speech sound integration has not been investigated before with a high temporal resolution method. However, in two recent ERP-studies, the auditory mismatch negativity (aMMN), known to reflect automatic auditory deviancy detection, was successfully employed to investigate the automaticity and timing properties of the influence of letters on low level speech sound processing (Froyen, Bonte, van Atteveldt, \& Blomert, 2009; Froyen, van Atteveldt, Bonte, \& Blomert, 2008). The aMMN is evoked between 100 and $250 \mathrm{msec}$ after stimulus onset when in a sequence of auditory stimuli a rarely presented sound (the deviant) deviates in one or more aspects from a frequently presented sound (the standard) (Näätänen, 1995; Schröger, 1998). The aMMN is an automatic and purely auditory deviance detection mechanism with a major source located in the auditory cortex (Ahlo, 1995; Giard, 1990). The aMMN evoked by the deviant speech sound /o/ violating the standard speech sound /a/ (auditory only experiment) was compared with the aMMN evoked by the deviant speech sound /o/ violating both the standard speech sound /a/ and the simultaneously presented standard letter "a" (audiovisual experiment) (Froyen, van Atteveldt, Bonte, \& Blomert, 2008). The aMMN amplitude evoked in the audiovisual experiment was enhanced in comparison with the aMMN amplitude in the auditory only experiment, presumably caused by the double deviation of the deviant speech sound from 
the standard speech sound and the standard letter. Considering the properties of the aMMN, this enhancement strongly points to early and automatic influences of letters on speech sound processing. Recently, the same MMN paradigm was successfully employed to investigate the influence of reading development on the time course and automaticity of letter - speech sound processing (Froyen, Bonte, van Atteveldt, \& Blomert, 2009), which validated this paradigm as a tool to investigate letter - speech sound processing passively and non-invasively.

Interestingly in this light, recently a number of studies have found converging evidence for the existence of a visual counterpart of the aMMN (Berti \& Schröger, 2003; Czigler, 2007; Czigler, Balazs, \& Pato, 2004; Heslenfeld, 2003; Maekawa et al., 2005; Tales, Newton, Troscianko, \& Butler, 1999). The visual MMN (vMMN) is described as a negativity measured at the occipital electrodes between 150 and 350 msec after the onset of an infrequent (deviant) visual stimulus in a sequence of frequently presented (standard) visual stimuli. The vMMN is suggested to have similar properties as the aMMN. It can be evoked pre-attentively and it reflects the use of a memory representation of regularities of visual stimulation (Czigler, 2007). The VMMN has been reported to be elicited by deviants differing in spatial frequency (Heslenfeld, 2003; Kenemans, Grent-'t Jong, \& Verbaten, 2003), line orientation (Astikainen, Ruusuvirta, Wikgen, \& Korjonen, 2007) and shape (Maekawa et al., 2005; Tales \& Butler, 2006; Tales, Haworth, Wilcock, Newton, \& Butler, 2008; Tales, Newton, Troscianko, \& Butler, 1999). The neural sources are suggested to reside in the extrastriate areas of the brain (Czigler, Balazs, \& Pato, 2004). The properties of the $\mathrm{VMMN}$ make it an appropriate tool to look for automatic influences of speech sounds on letter processing and investigate the time course of these influences.

In the present study the $\mathrm{VMMN}$ evoked by a deviant letter in a visual-only experiment (Figure $1 \mathrm{~A}$ ) is compared with the $\mathrm{vMMN}$ evoked by the same deviant letter accompanied by a standard speech sound (audiovisual, Figure 1B). As a control for the letter-specificity of potential auditory effects on the $\mathrm{VMMN}$, a second deviant, the non-letter '*', was included. First, we predict a typical vMMN in the visual-only experiment, probably stronger for the non-letter deviant because it deviates more in basic visual features from the standard visual stimulus. Second, if there is, due to integration, an automatic influence of the speech sound on letter processing, this is expected to be reflected in an effect of experiment (visual versus audiovisual) on the vMMN amplitude in analogy to the aMMN studies (Froyen, Bonte, van Atteveldt, \& Blomert, 2009; Froyen, van Atteveldt, Bonte, \& Blomert, 2008). Third, if this influence is letter-specific, we expect a differential effect of the speech sound on the vMMN evoked by the letter versus the non-letter, reflected in an interaction between experiment (visual versus audiovisual) and condition (letter versus nonletter). 


\section{Material and Methods}

\section{Participants}

Twelve subjects participated in the visual-only experiment, (10 female, range 18 to 28 , mean age 21.65 years) and twelve subjects participated in the audiovisual experiment, (9 female, range 20 to 33, mean age 24.69 years). Subjects were randomly assigned to either the visual only or the audiovisual experiment. Subjects were all students who were paid for their participation or received course credits per experiment. Informed consent was obtained from all the subjects, according to the approval by the Ethical Committee of the Faculty of Psychology and Cognitive Neuroscience, Maastricht.

\section{Stimuli and procedure}

Stimuli were the natural speech sound /a/ (384 ms), the visually presented single letters ' $a$ ' and ' $o$ ', and a visually presented non-letter '*'. The speech sound /a/ was digitally recorded (sampling rate $44.1 \mathrm{kHz}, 16$ bit quantization) from a female speaker. Recordings were band-pass filtered (180-10.000 Hz) and resampled at $22.05 \mathrm{kHz}$ and matched for loudness with Praat software (Boersma \& Weenink, 2002). The sounds were presented binaurally through loudspeakers at about $65 \mathrm{~dB}$ SPL. Letters and non-letters were presented in white on a black background in the centre of a computer screen for $500 \mathrm{~ms}$ (visual angle: 1.2 by 0.8 degrees). Between the presentations of the visual stimuli a white fixation cross was presented in the centre of the screen.

\section{A) Visual experiment}

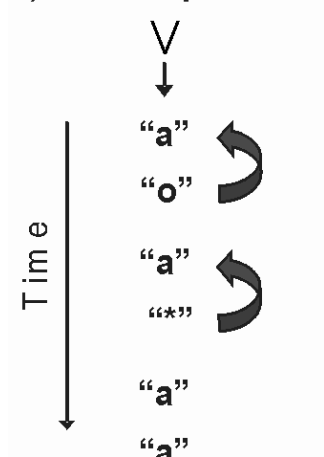

\section{B) Audiovisual experiment}

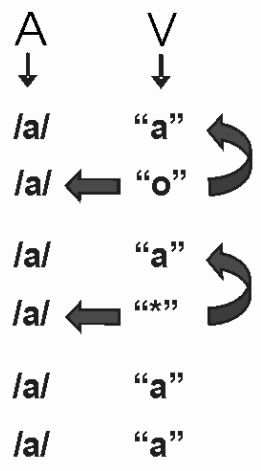

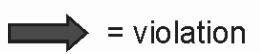

Figure 1. Visualization of the design in the visual (A) and the audiovisual experiment (B). ' $A$ ' stands for auditory stimulus, ' $V$ ' stands for visual stimulus. The ' $o$ ' was the letter deviant violating the previously presented standard letter ' $a$ ', and in the audiovisual experiment also the simultaneously presented speech sound /a/. The '*' was the non-letter deviant included to control for letter-specificity. 
In both experiments, subjects saw either the standard letter ' $a$ ' $(80 \%)$, the letter deviant ' $\mathrm{o}$ ' $(10 \%)$ or the non-letter deviant ' ${ }^{\prime * \prime}(10 \%)$. To ensure that subjects were at all times focusing the screen, we presented 10 non-related salient color pictures per run. Subjects had to press a button when they saw the picture. During the visual experiment there was no auditory stimulation (Figure $1 \mathrm{~A}$ ). In the audiovisual experiment a speech sound /a/ was presented simultaneously with the visual stimuli (Figure 1B). We also presented a speech sound deviant /o/ (10\%), but this condition is irrelevant for the present research aim and is reported elsewhere (Froyen, van Atteveldt, Bonte, \& Blomert, 2008). During the trials of interest for the present study, the speech sound /a/ was always presented simultaneously with the visual stimulus. In each experiment, there were 4 blocks with 534 trials. Trial length was $1250 \mathrm{~ms}$, the inter-trial interval was $750 \mathrm{~ms}$.

\section{Recording and analysis}

EEG data were recorded with NeuroScan 4.2 from 0.01 to $50 \mathrm{~Hz}$ with a sampling rate of $250 \mathrm{~Hz}$ in a sound-attenuating and electrically shielded room from 30 electrode positions (Extended International 10-20 system) relative to a nose reference. Eye-movements and blinks were measured with bipolar VEOG/HEOG channels. All electrode impedance levels (EEG and EOG) were kept below $5 \mathrm{k} \Omega$. EEG data were epoched from -50 to $800 \mathrm{~ms}$ relative to trial onset, baseline corrected (50 ms pre stimulus interval), and $1-30 \mathrm{~Hz}$ band pass filtered with NeuroScan 4.2. Epochs containing data exceeding a maximum voltage criterion of $75 \mu \mathrm{V}$ were rejected. Standards immediately following deviants were not included in the analysis, as were the trials with target pictures. The raw EEG data were corrected for vertical eyemovements (i.e. blink artifacts). In the visual experiment we retained 176 (88\%) letter standard, 175 (88\%) letter deviant and 172 (86\%) non-letter deviant trials and in the audiovisual experiment we retained 184 (92\%) standard, 185 (93\%) letter deviant and 186 (93\%) non-letter deviant trials. Epochs were averaged separately for each condition and each participant. Difference waves were calculated by subtracting the ERPs to the standard condition from ERPs to the deviant condition.

Statistical analysis was performed on 11 electrodes covering the fronto-central ( $\mathrm{Fz}$, $\mathrm{Cz}, \mathrm{FC} 3, \mathrm{FC} 4)$, temporal (T3 and T4), parietal (CP3 and CP4), and occipital (Oz, 01 and $\mathrm{O} 2$ ) regions of the brain to investigate the visual $\mathrm{MMN}$. As in previous visual oddball studies (Alho, Woods, Algazi, \& Naatanen, 1992; Kimura, Katayama, Ohira, \& Schröger, 2009; Maekawa et al., 2005; Tales \& Butler, 2006; Tales, Haworth, Wilcock, Newton, \& Butler, 2008; Wei, Chan, \& Luo, 2002), there appeared to be two time windows of interest in which the deviants differed from the standard: An early time window between 150 and 220 ms after stimulus onset (deviant related negativity 1 or DRN1) and a later time window between 240 to 320 ms (deviant related negativity 2 or DRN2). Each time window was analyzed separately. From the grand 
average as well as the difference waveforms, we derived two dependent variables per time window for each electrode: (I) the latency measured at the maximum peak amplitude in the latency range from 150 to $250 \mathrm{~ms}$ post stimulus onset for the early time window and from 250 to 350 ms post stimulus onset for the later time window and (II) the amplitude measured as the mean amplitude across 50 ms centered around the individual peak latency. Both measures were derived separately for each subject, condition, electrode and time window. We used the mean area amplitude rather than the peak amplitude as it is considered a more reliable measure. Consequently, in the present study, the term amplitude always refers to the mean area amplitude. First, a repeated measures ANOVA with electrode sites ( $F z, C z, P z, O z$, FC3, FC4, T3, T4, O1, O2, CP3 and CP4) and condition (standard letter, deviant letter and deviant non-letter) as within subject variables and experiment (visual only and audiovisual) as between subject variable was performed. If there was an interaction with electrode, the analysis on the initial 11 electrodes was followed by a detailed analysis on the occipital electrodes (Oz, $\mathrm{O} 1$ and $\mathrm{O} 2)$, where the deviancy-effect was expected to be most prominent (Czigler, Balazs, \& Pato, 2004). Firstly, amplitude and latency measures of the grand average waveforms were analyzed to check for an effect of speech sounds on letter processing. Secondly, difference waveforms were analyzed. All reported $p$-values from the repeated measures ANOVA were greenhouse-Geisser corrected. All post-hoc analyses were Bonferroni corrected.

\section{Results}

\section{General description of ERP waveforms and determination of windows of interest}

A positive (P1) - negative (N1) - positive (P2) deflection was observed in the grand average waves to standard, letter deviant and non-letter deviant stimuli in both experiments, indicating typical exogenous components (Figure 2). Difference waveforms (letter deviant minus standard and non-letter deviant minus standard trials) are shown in Figure 3. Most pronounced in the difference waves at the occipital electrodes ( $\mathrm{Oz}, \mathrm{O} 1$ and $\mathrm{O} 2$ ), a deviant related negativity (DRN) was observed between 150 and 320 ms after stimulus onset, which is in line with previously reported latencies (Czigler, 2007; Pazo-Alvarez, Cadaveira, \& Amenedo, 2003). As in previous visual MMN studies (Alho, Woods, Algazi, \& Naatanen, 1992; Kimura, Katayama, Ohira, \& Schröger, 2009; Maekawa et al., 2005; Tales \& Butler, 2006; Tales, Haworth, Wilcock, Newton, \& Butler, 2008; Wei, Chan, \& Luo, 2002), there appeared to be two time windows of interest: An early time window between 150 and $220 \mathrm{~ms}$ after stimulus onset (DRN1) and a later time window between 240 to $320 \mathrm{~ms}$ (DRN2). 
Table 1. Mean area amplitudes and one sample t-test values per occipital electrode $(\mathrm{Oz}, \mathrm{O} 1$ and $\mathrm{O} 2)$ for both time windows of interest and per condition in the visual only experiment.

\begin{tabular}{lllll}
\hline Visual experiment & \multicolumn{2}{l}{$\begin{array}{l}\text { grand average waves } \\
\text { amplitude }\end{array}$} & \multicolumn{2}{c}{ one sample t-test } \\
& & & $\mathrm{t}$ & $p$ \\
\hline DRN1: & & & -5.2 & $<.001$ \\
Letter & $\mathrm{Oz}$ & -1.96 & -5.2 & $<.001$ \\
& $\mathrm{O} 1$ & -2.19 & -5.1 & $<.001$ \\
& $\mathrm{O} 2$ & -2.26 & -2.9 & $=.015$ \\
Non-letter & $\mathrm{Oz}$ & -1.77 & -3.0 & $=.011$ \\
& $\mathrm{O} 1$ & -1.93 & -3.1 & $=.011$ \\
& $\mathrm{O} 2$ & -2.01 & & \\
DRN2: & & & -5.6 & $<.001$ \\
Letter & $\mathrm{Oz}$ & -1.91 & -4.9 & $<.001$ \\
& $\mathrm{O} 1$ & -1.94 & -5.7 & $<.001$ \\
& $\mathrm{O} 2$ & -1.89 & -6.7 & $<.001$ \\
Non-letter & $\mathrm{Oz}$ & -3.17 & -6.4 & $<.001$ \\
& $\mathrm{O} 1$ & -3.21 & -6.9 & $<.001$ \\
\hline
\end{tabular}

To assure a typical vMMN in the visual only experiment, a one sample t-test of the difference wave mean area amplitudes was performed per occipital electrode $(\mathrm{Oz}$, $\mathrm{O} 1$ and 02) per condition (letter and non-letter) and per time window (DRN1 and DRN2). All difference wave mean area amplitudes differed significantly from zero (Table1), justifying a closer look at the data to check for an effect of speech sounds on the vMMN to letter deviants and/or non-letter deviants.

\section{DRN1 time window}

\section{Grand average waves}

Mean area amplitudes of grand average waves in the DRN1 time window were first analyzed using a 3 (condition: standard letter, deviant letter and deviant non-letter) x 11 (electrode sites: Fz, Cz, Pz, Oz, FC3, FC4, T3, T4, O1, O2, CP3 and CP4) repeated measures ANOVA with experiment (visual and audiovisual experiment) as a between subject variable. There was no significant three-way interaction, $F(20,440)=$ $.89, p=.445$, but an interaction between condition and electrode, $F(20,440)=$ $10.14, p<.001$. Further analysis on the three occipital electrodes where the deviancy-effect was expected to be most prominent (Czigler, Balazs, \& Pato, 2004) revealed no significant three-way interaction, $F(4,88)=2.09, p=.140$, no significant interaction between condition and electrode sites, $F(4,88)=1.22, p=.304$, no significant interaction between experiment and electrode sites, $F(2,44)<1$, and no significant interaction between condition and experiment, $F(2,44)=1.60, p=.218$. There was a significant main effect of condition, $F(2,44)=16.16, p<.001$. Pairwise comparisons revealed a significant difference between the standard and the nonletter deviant condition, $p<.001$, and between the letter deviant and the non-letter 
deviant condition, $p=.001$, but not between the standard and the letter deviant condition, $p=1$. Amplitudes to the non-letter deviant were more negative in comparison with amplitudes to the letter deviant and the standard letter. However, there was no main effect of experiment, $F(1,22)<1$, indicating no effect of speech sounds on letter or non-letter processing in this early time window.

A repeated measures ANOVA of the latencies of the grand average waves' peaks with condition ( 3 ) and electrode sites (11) as within subject factors and experiment (2) as a between subject factor revealed no significant three-way interaction, $F(20$, $440)<1$, but a marginally significant two-way interaction between electrode sites and experiment, $F(10,220)=2.32, p=.059$. Further analysis on only the three occipital electrodes revealed no significant three-way interaction, $F(4,88)=1.31, p=$ .277 , no significant interaction between condition and electrode sites, $F(4,88)<1$, no significant interaction between experiment and electrode sites, $F(2,44)=1.04, p$ $=.342$, and no significant interaction between condition and experiment, $F(2,44)=$ $1.60, p=.218$. There was no significant main effect of experiment, $F(1,22)<1$, but a significant main effect of condition, $F(2,44)=11.14, p<.001$. Pairwise comparisons revealed a significant difference between the standard and the letter deviant condition, $p=.011$, and between the letter deviant and the non-letter deviant condition, $p=.001$, but not between the standard and the non-letter deviant condition, $p=1$. The peak latency in the letter deviant condition was up to $30 \mathrm{~ms}$ delayed in comparison with the peak latency of the non-letter deviant and the standard letter. However, again there was no significant main effect of experiment, $F(1,22)<1$. 


\section{Grand average waves}

A. Visual experiment

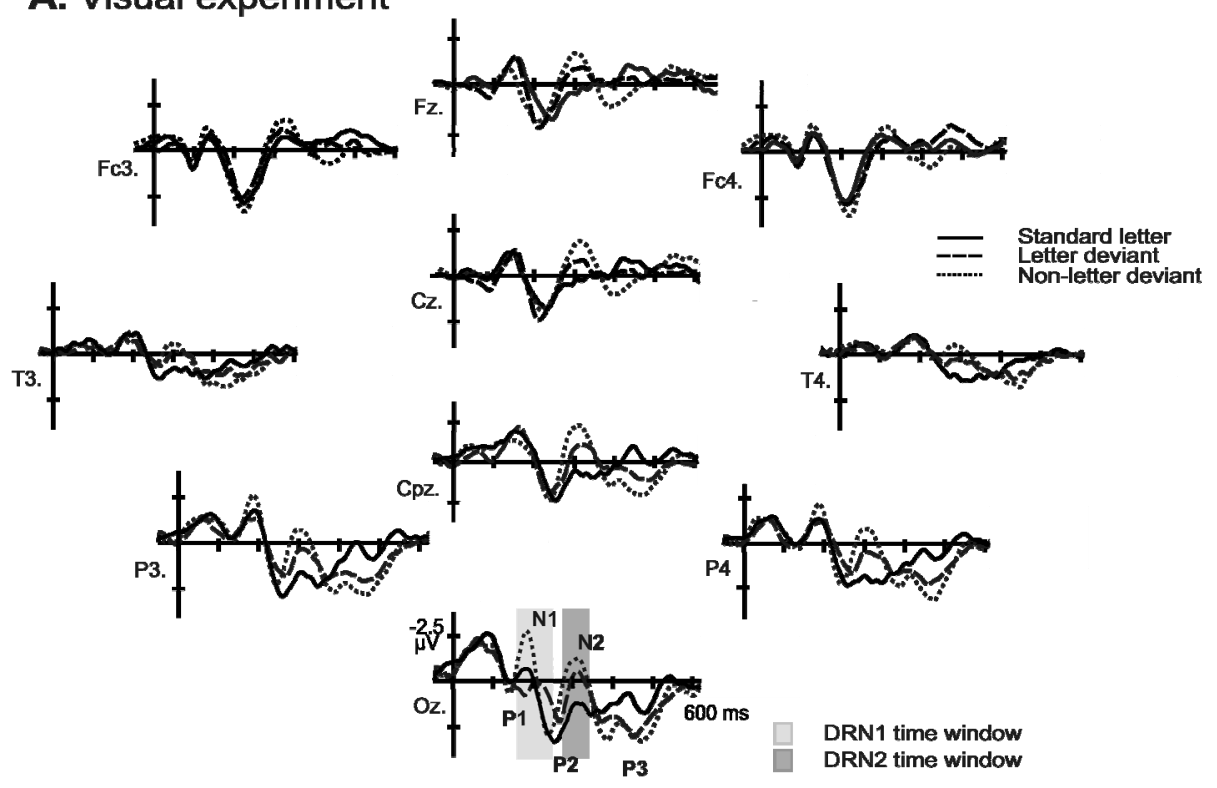

B. Audiovisual experiment

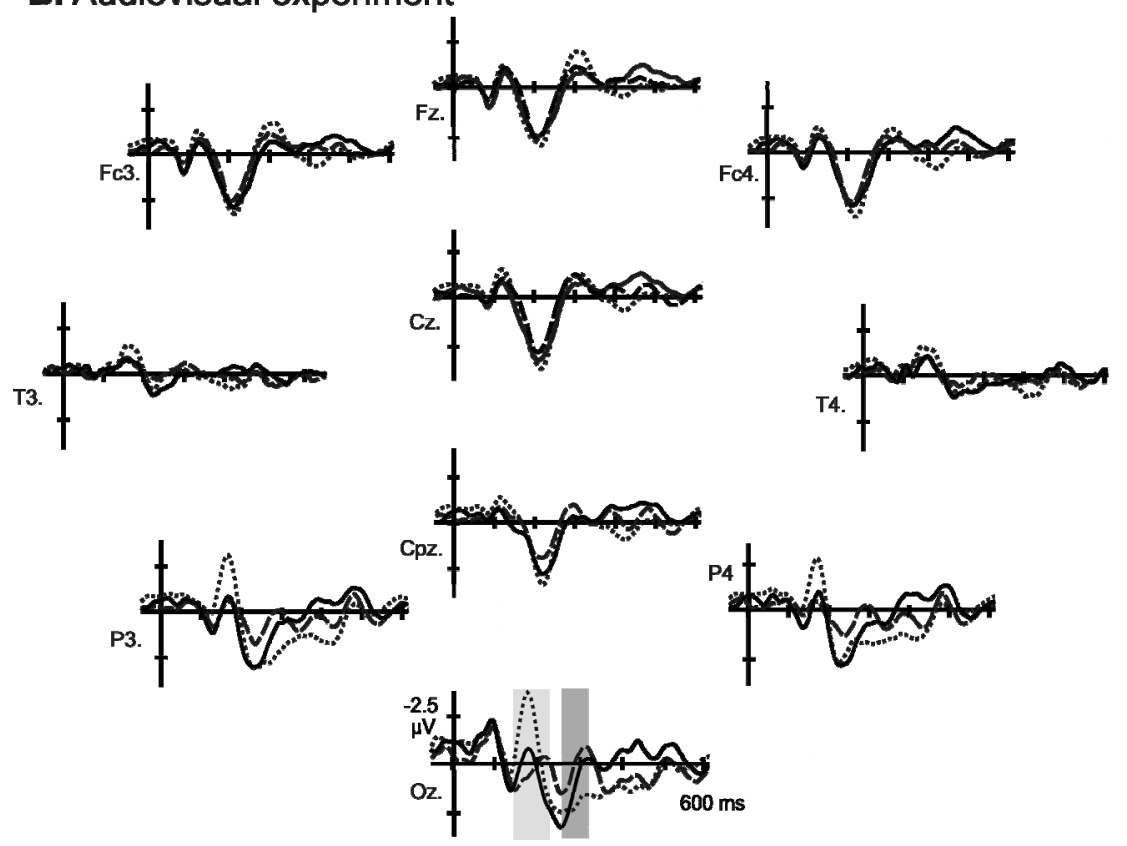

Figure 2. Grand-average waves observed at ten electrodes ( $\mathrm{Fz}, \mathrm{Fc} 3, \mathrm{Fc} 4, \mathrm{Cz}, \mathrm{T} 3, \mathrm{~T} 4, \mathrm{Cpz}, \mathrm{P} 3, \mathrm{P} 4$ and $\mathrm{Oz}$ ) in the visual experiment $(A)$ and the audiovisual experiment $(B)$. 


\section{Difference waves}

Since there was no effect of experiment on either the mean area amplitude or latency of the grand averages waves in this early time window, no effect of experiment on the mean area amplitudes of the difference waves is to be expected. Analysis of the mean area amplitudes of the difference waves using repeated measures ANOVA with two within subject factors (condition: Letter and non-letter deviant, and occipital electrode sites: $01, \mathrm{Oz}$ and 02 ) and one between subject factor (visual and audiovisual experiment) revealed no significant three-way interaction between condition, electrode sites and experiment, $F(2,44)<1$, no significant twoway interaction between condition and electrode, $F(2,44)<1$, no significant twoway interaction between electrode and experiment, $F(2,44)=2.51, p=.098$, and no significant two-way interaction between condition and experiment, $F(2,2)=5.96, p$ $=.334$, indicating no different effect of experiment on either letter or non-letter processing. Moreover, there was no main effect of experiment, $F(2,44)=1.09, p=$ .306 , indicating no effect of experiment regardless of condition. These results corroborate the results obtained from the grand average waves' analysis.

\section{DRN2 time window}

\section{Grand average waves}

Mean area amplitudes of grand average waves in the DRN2 time window were first analyzed using repeated measures ANOVA ( 3 conditions $\times 11$ electrode sites $\times 2$ experiments). There was a significant three-way interaction, $F(20,440)=9.09, p<$ .001. Further analysis was therefore performed on the three occipital electrodes where the deviancy-effect was expected to be most prominent (Czigler, Balazs, \& Pato, 2004). This $3 \times 3 \times 2$ analysis revealed no significant three-way interaction, $F(4$, $88)=1.18, p=.32$, but a two-way interaction between condition and experiment, $F(2,44)=15.63, p<.001$, indicating differential influences of the speech sound on letter and non-letter processing. Analysis per condition revealed no difference between the two experiments considering the amplitude of the standard, $F(1,22)=$ 2.26, $p=.147$ and the letter deviant condition, $F(1,22)=1.17, p=.29$, but a significantly less negative amplitude to the non-letter deviant in the audiovisual experiment in comparison with the visual experiment, $F(1,22)=7.98, p=.010$, indicating a suppression of non-letter processing when the non-letter was presented simultaneously with a speech sound (Table 2). 


\section{Difference waves}

A. Visual experiment

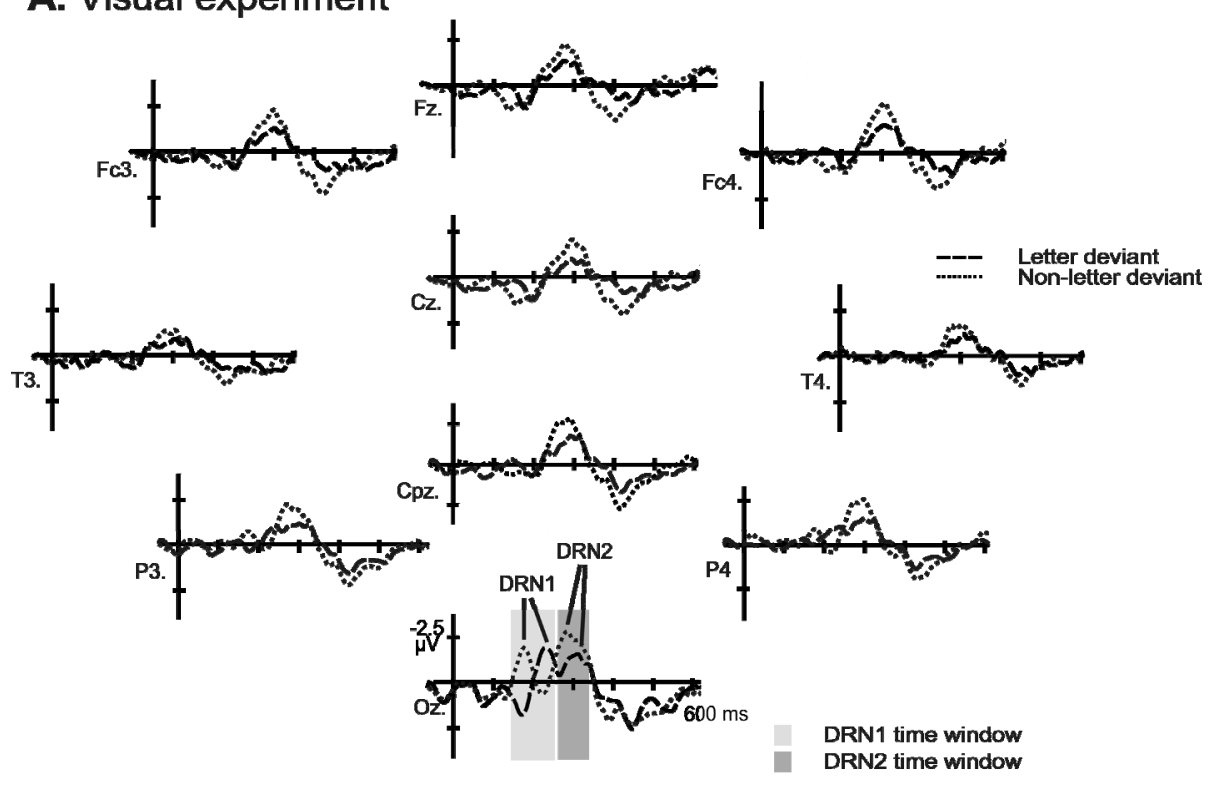

B. Audiovisual experiment
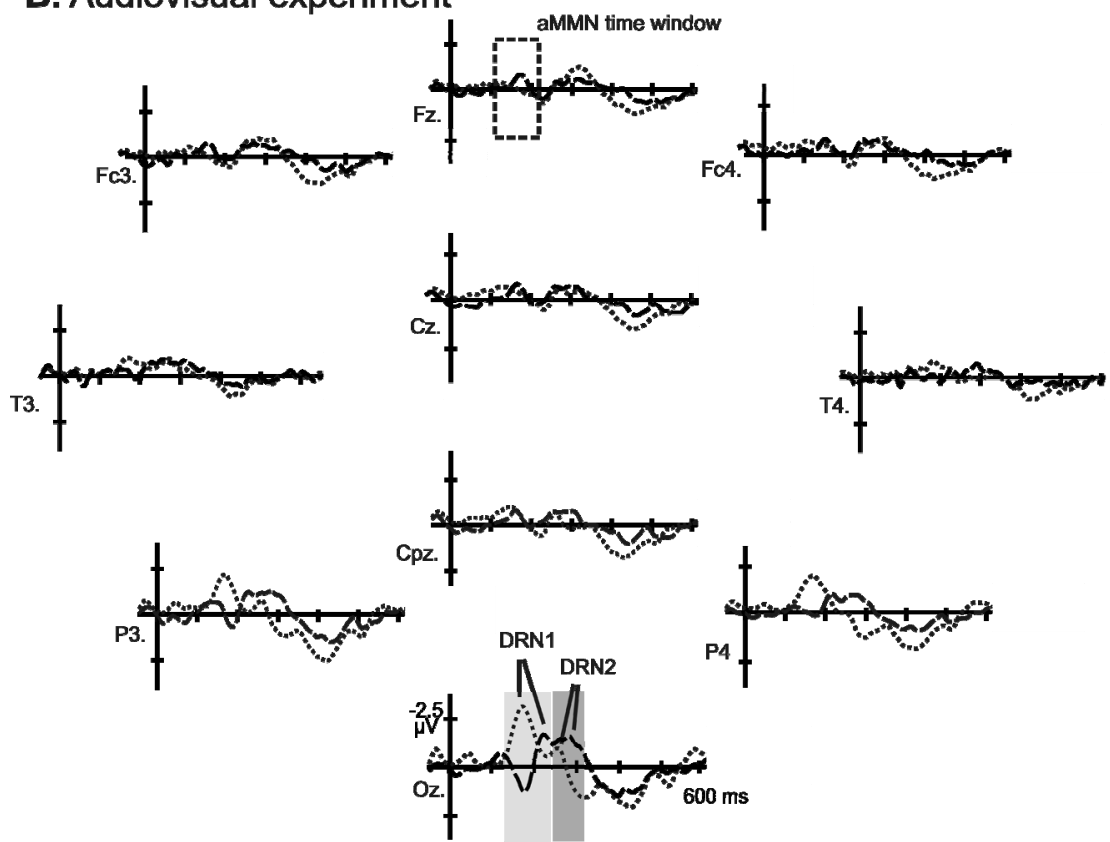

Figure 3. Difference waves observed at ten electrodes ( $\mathrm{Fz}, \mathrm{Fc} 3, \mathrm{Fc} 4, \mathrm{Cz}, \mathrm{T} 3, \mathrm{~T} 4, \mathrm{Cpz}, \mathrm{P} 3, \mathrm{P} 4 \mathrm{and} \mathrm{Oz}$ ) in the visual experiment $(A)$ and the audiovisual experiment $(B)$. 
A 3 by 11 by 2 repeated measures ANOVA of the latencies revealed a marginally significant three-way interaction, $F(20,440)=1.98, p=.052$. A subsequent analysis with only the three occipital electrodes revealed no three-way interaction, $F(4,88)<$ 1 , and no two-way interactions; $F(4,88)<1$, for condition $x$ electrode sites, $F(4,88)$ $<1$, for electrode sites $\mathrm{x}$ experiment, and $F(2,44)=2.99, p=.060$, for condition $\mathrm{x}$ experiment. There was a main effect of condition, $F(2,44)=4.17, p=.022$. Post hoc comparisons revealed only a difference between the standard and the letter deviant condition, $p=.032$, and not between the standard and the non-letter deviant condition, $p=.214$, or the letter and the non-letter condition, $p=.956$. There was no significant main effect of experiment, $F(1,22)<1$, indicating no effect of speech sounds on latency of either letter or non-letter processing.

Table 2. Mean area amplitude and peak latency measures of the grand average waves and the difference waves with standard errors of the mean for DRN1 and DRN2 averaged over the three occipital electrodes $\mathrm{Oz}, \mathrm{O} 1$ and $\mathrm{O} 2$.

\begin{tabular}{|c|c|c|c|c|}
\hline \multirow[t]{2}{*}{ Condition } & \multicolumn{2}{|c|}{ grand average waves } & \multicolumn{2}{|c|}{ difference waves } \\
\hline & Amplitude & latency & amplitude & latency \\
\hline \multicolumn{5}{|l|}{ DRN1: } \\
\hline \multicolumn{5}{|l|}{ Visual experiment } \\
\hline Standard letter & $-1.2 \pm 0.6$ & $172 \pm 6$ & & \\
\hline Letter deviant & $-1.2 \pm 0.5$ & $188 \pm 9$ & $-2.1 \pm 0.4$ & $217 \pm 4$ \\
\hline Non-letter deviant & $-2.8 \pm 0.8$ & $173 \pm 7$ & $-1.9 \pm 0.4$ & $168 \pm 6$ \\
\hline \multicolumn{5}{|l|}{ Audiovisual experiment } \\
\hline Standard letter & $-1.1 \pm 0.6$ & $173 \pm 6$ & & \\
\hline Letter deviant & $-0.7 \pm 0.6$ & $203 \pm 8$ & $-2.6 \pm 0.8$ & $204 \pm 8$ \\
\hline Non-letter deviant & $-3.9 \pm 1.1$ & $168 \pm 5$ & $-3.2 \pm 0.7$ & $155 \pm 6$ \\
\hline \multicolumn{5}{|l|}{ DRN2: } \\
\hline \multicolumn{5}{|l|}{ Visual experiment } \\
\hline Standard letter & $0.9 \pm 0.5$ & $319 \pm 8$ & & \\
\hline Letter deviant & $0.02 \pm 0.4$ & $294 \pm 7$ & $-1.9 \pm 0.4$ & $291 \pm 6$ \\
\hline Non-letter deviant & $-1.0 \pm 0.7$ & $292 \pm 8$ & $-3.2 \pm 0.5$ & $277 \pm 7$ \\
\hline \multicolumn{5}{|l|}{ Audiovisual experiment } \\
\hline Standard letter & $0.02 \pm 0.3$ & $307 \pm 6$ & & \\
\hline Letter deviant & $-0.6 \pm 0.4$ & $295 \pm 6$ & $-2.4 \pm 0.8$ & $263 \pm 6$ \\
\hline Non-letter deviant & $1.6 \pm 0.7$ & $311 \pm 7$ & $-0.6 \pm 0.6$ & $243 \pm 6$ \\
\hline
\end{tabular}

\section{Difference waves}

The mean area amplitudes of the difference waves were analyzed using repeated measures ANOVA with two within subject factors (condition: Letter and non-letter deviant, and occipital electrode sites: $\mathrm{O} 1, \mathrm{Oz}$ and 02 ) and one between subject factor: visual and audiovisual experiment. There was a significant three-way interaction between condition, electrode sites and experiment, $F(2,44)=3.59,=.045$. Because the three-way interaction indicated differential effects of the visual versus the audiovisual experiment for the letter versus the non-letter deviant, a two way repeated measures ANOVA (electrode and experiment) was conducted per condi- 
tion. For the letter deviant no main effect of experiment was found, $F(1,22)<1$. For the non-letter deviant, however, difference wave amplitudes in the audiovisual experiment were significantly lower in comparison with those in the visual only experiment, $F(1,22)=11.09,=.003$ (Figure 4), corroborating the results obtained from the grand average waves' analysis.

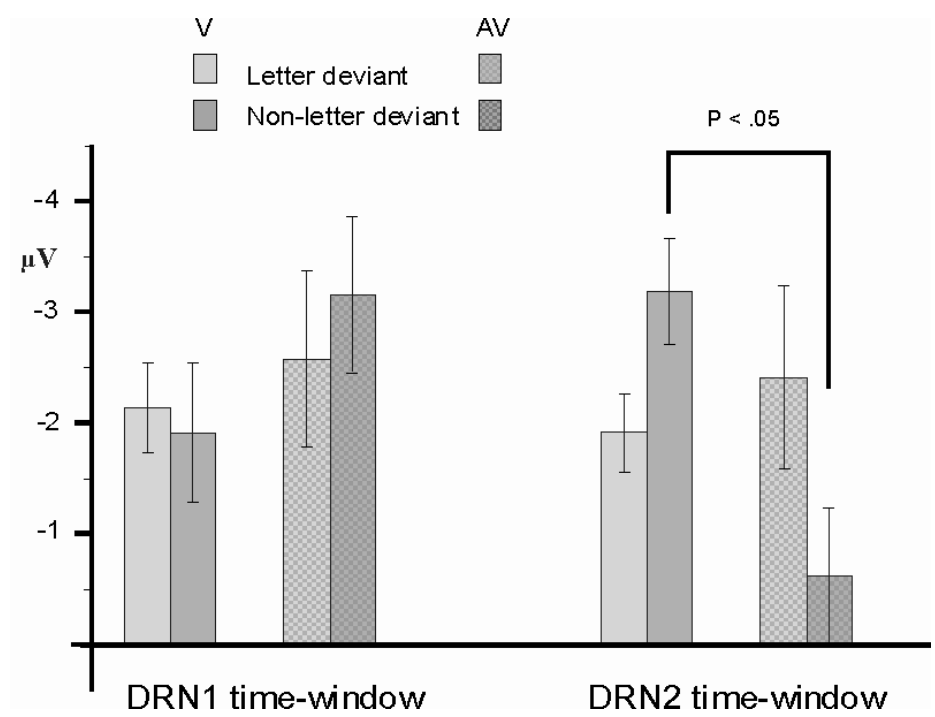

Figure 4. Mean area amplitudes of the difference waves to letters and non-letters in DRN1 time window (left) and DRN2 time window (right) obtained in the visual experiment (V) versus the audiovisual experiment (AV), averaged over the three occipital electrodes $\mathrm{Oz}, \mathrm{O} 1$ and $\mathrm{O} 2$.

\section{Discussion}

To gain insight in the involvement of low level visual processing during letter speech sound integration, we investigated the automaticity and time-course of the influence of speech sounds on letter processing. The visual counterpart of the auditory MMN was measured to letter and non-letter deviants presented either in isolation (visual only experiment) or simultaneously with speech sounds (audiovisual experiment). In the visual only experiment, a posterior negativity in both the letter and non-letter difference waves was observed between 150 and 320 ms, supporting the literature in favour of a deviance detection mechanism in the visual modality (Czigler, 2007; Pazo-Alvarez, Cadaveira, \& Amenedo, 2003). In agreement with previous vMMN-studies (Alho, Woods, Algazi, \& Naatanen, 1992; Kimura, Katayama, Ohira, \& Schröger, 2009; Maekawa et al., 2005; Tales \& Butler, 2006; Tales, Haworth, Wilcock, Newton, \& Butler, 2008; Wei, Chan, \& Luo, 2002), we found two time windows of interest: An early time window between 150 and $220 \mathrm{~ms}$ after stimulus onset and a second time window between 240 and 320 ms. Kimura and 
coauthors (2009) recently investigated the difference in functionality of the two negativities: the early negativity (DRN1) was suggested to reflect a refractory effect, i.e., lower adaptation of neurons specifically responsive to features of the infrequent deviant stimulus, while the later negativity (DRN2) was suggested to reflect a memory-comparison-based deviancy detection effect and to represent the visual counterpart of the auditory MMN.

There were no influences of the speech sound on the morphology, amplitude or latency of DRN1 to letter or non-letter processing in the early time window. However, the non-letter deviant evoked a higher negativity in comparison with the letter deviant in both experiments. This might be explainable by the hypothesized functionality of the early negativity (Kimura, Katayama, Ohira, \& Schröger, 2009). That is because the non-letter deviant, ' ${ }^{\prime}$ ', deviates more in basic visual features from the standard letter, ' $a$ ', in comparison with the letter deviant, ' $o$ ', which resembles the standard letter in basic visual features. Consequently, the non-letter deviant shares a lower amount of responsive neurons with the standard letter, leaving the neuronal population that is specifically responsive to the non-letter deviant largely unadapted during the presentation of the standard letter.

More interestingly, in the DRN2 time window, speech sounds had a differential effect on letter and non-letter processing. While the speech sound had no effect on either standard or deviant letter processing, the grand average and difference wave amplitude to non-letter processing decreased significantly if accompanied with a speech sound. The latency of this second time window, between 240 and $320 \mathrm{~ms}$ after stimulus onset, actually coincides with the latency of the letter - speech sound congruency effect, i.e. between 250 and $500 \mathrm{~ms}$, reported in the MEG-study by Herdman and coauthors (2006). We therefore propose that the latency as well as the broad topographical distribution (Figure 3 ) of this late negativity effect point to a content-related processing mechanism.

However, this late effect of speech sounds on letter processing is not a reversal of the effects of letters on speech sounds that we reported previously (Froyen, Bonte, van Atteveldt, \& Blomert, 2009; Froyen, van Atteveldt, Bonte, \& Blomert, 2008). In these studies, we found a significantly increased amplitude of the auditory MMN in the audiovisual experiments in response to the double, cross-modal violation of the deviant letter with respect to both the standard letter and the standard speech sound. In the present study, the grand average waves to the standard and deviant letter as well as the difference waves to the deviant letter did not differ between the visual and the audiovisual experiment. This indicates that speech sounds do not automatically influence standard or deviant letter processing in a way comparable to the automatic modulation of speech sound processing by letters. The present results therefore shed more light on the role of low level visual processing during letter - speech sound integration. Whereas low level auditory processing is automatically involved in letter - speech sound integration (Froyen, van Atteveldt, 
Bonte, \& Blomert, 2008), this does not seem to hold for low level visual processing as was also indicated by previous $\mathrm{fMRI}$ studies using a passive task design (Van Atteveldt, Formisano, Blomert, \& Goebel, 2007; Van Atteveldt, Formisano, Goebel, \& Blomert, 2004). Despite the use of a high temporal resolution method in the present study no automatic influences of speech sounds on letter processing were found, thus supporting an asymmetry in the involvement of low level auditory and visual areas during letter - speech sound integration.

It might be that only when subjects have to actively process letter - speech sound pairs, interaction effects in low level visual areas can be recorded with a high temporal resolution method (Herdman et al., 2006). The task used in the MEG study by Herdman and colleagues required the subjects to make a congruency decision on the presented letters and speech sounds. This active task design promotes both congruent and incongruent letters to immediate relevance, thereby not only recruiting low level auditory areas, but also involving low level visual processing areas. Interestingly, in an ERP-study with newly learned meaningless audiovisual stimuli, similar task effects were reported (Fort \& Giard, 2004). During a stimulus identification task, requiring active processing of the auditory and the visual features of the stimulus, integration effects in visual areas were observed in three time windows, i.e. between $50-100,100-150$ and $150-200$ ms. However, when subjects were asked to simply detect each stimulus, not requiring active processing of the stimulus properties, only early interactions $(50-100 \mathrm{~ms})$ were observed. Alternatively, attention might play a crucial role in finding cross-modal integration effects in the low level visual system. In an audiovisual ERP-study with, again, meaningless stimuli in which attention was systematically manipulated, integration effects on the early P50 component amplitude were strongly reduced during the unattended condition (Talsma, Doty, \& Woldorff, 2007). A superadditive integration effect was observed when both auditory and visual stimuli were simultaneously attended, while in the unattended condition the audiovisual P50 was smaller than the sum of the two unimodal P50s. In sum, it seems that processing arbitrarily linked audiovisual stimuli does not automatically involve low level visual processing. Note that these studies are either MEG (Herdman et al., 2006) or EEG (Fort \& Giard, 2004; Talsma, Doty, \& Woldorff, 2007) studies, supporting our suggestion that the usage of a high temporal resolution method might be critical for revealing the involvement of low level visual processing areas during audiovisual integration of arbitrarily related stimuli, like letters and speech sounds.

These indications for an asymmetric involvement of low level sensory areas during letter - speech sound processing, and presumably also during audiovisual processing of other arbitrarily linked stimuli, are in contrast with the indications for a symmetric involvement of both low level sensory cortices during audiovisual speech processing (Calvert et al., 1999; Calvert, Campbell, \& Brammer, 2000; Macaluso, George, Dolan, Spence, \& Driver, 2004). A plausible explanation for the difference in 
the involvement of the low level visual areas is that audiovisual speech, but not letter - speech sound processing, is a natural product of phylogenetic and ontogenetic spoken language development. Consequently, a naturally evolved neural system for processing and integrating letters and speech sounds is unlikely to exist (Gleitman and Rozin 1977; Liberman 1992). It has been suggested that reading parasites on speech (Mattingly 1972), making it plausible that information from the parasitic mechanism, in this case visual letter information, travels to the speech processing system and not vice versa (see also van Atteveldt et al., 2007a). Alternatively, the nature of the link between the two modalities of a stimulus may be critical for the automaticity of the involvement of both low level sensory cortices (Calvert, 2001). When we see and hear speech being spoken, the auditory speech signal shares time varying aspects with the concurrent lip movements (Amedi, von Kriegstein, Van Atteveldt, Beauchamp, \& Naumer, 2005; Calvert, Brammer, \& Iversen, 1998; Munhall \& Vatikiotis-Bateson, 1998). These shared time varying aspects constitute a strong natural cross-modal binding factor. Letters, however, are culturally defined symbols without any natural relation with their corresponding speech sounds. A recent investigation of the development of letter - speech sound processing indicated that it takes years of reading instruction before letter - speech sound pairs are automatically integrated (Froyen, Bonte, van Atteveldt, \& Blomert, 2009), which stands in sharp contrast with the indications for audiovisual speech integration very early in development (Burnham \& Dodd, 2004). The latter more general explanation hypothesizes that our neural system is well adapted to integrate naturally linked cross-modal properties of a stimulus, while arbitrarily linked audiovisual properties probably recruit a different neural mechanism.

Another indication for a different neural mechanism for letter - speech sound integration is the fact that the letter deviant in the present study does not evoke an auditory MMN when presented simultaneously with speech sounds. Previous audiovisual $\mathrm{MMN}$ studies revealed that a visual deviant can evoke an auditory MMN, even if the physical properties of the auditory stimulus remain unaltered (Colin et al., 2002; de Gelder, Bocker, Tuomainen, Hensen, \& Vroomen, 1999; Möttönen, Krause, Tiippana, \& Sams, 2002; Sams et al., 1991; Stekelenburg, Vroomen, \& de Gelder, 2004). In order to check for this, we compared the negativities to the letter deviant, observed in a typical aMMN time window at the frontocentral electrodes, in the present audiovisual experiment (see $\mathrm{Fz}$ in figure $3 \mathrm{~B}$ ) with the aMMN evoked by a speech sound deviant in a previous auditory only experiment (Froyen, van Atteveldt, Bonte, \& Blomert, 2008). This comparison revealed that the morphology of the difference waves in the audiovisual experiment closely resembled those in the visual only experiment of the present study and not the aMMN in the previous auditory only experiment. The similarities between the difference waves at the frontocentral electrodes in the aMMN time window evoked in both the visual and the audiovisual experiment indicate that the observed negativities probably 
reflect visual evoked potentials from occipital generators, instead of visual influences on auditory processing. The letter deviants in the present experiment thus did not invoke an aMMN. As pointed out by Besle and coauthors, all studies in which an aMMN was evoked by merely deviating the visual part of an audiovisual stimulus used an auditory illusion (Besle, Fort, \& Giard, 2005). An aMMN has been evoked by the McGurk-illusion (Colin et al., 2002; Möttönen, Krause, Tiippana, \& Sams, 2002; Sams et al., 1991), the ventriloquist-illusion (Stekelenburg, Vroomen, \& de Gelder, 2004), and in a face-voice emotion identification study (de Gelder, Bocker, Tuomainen, Hensen, \& Vroomen, 1999). Our results support the suggestion that an illusion is necessary to evoke an aMMN by merely deviating the visual part in an audiovisual stimulus (Besle, Fort, \& Giard, 2005). The present evidence that a letter cannot evoke an aMMN furthermore supports the suggested asymmetric involvement of low level visual processing during passive letter - speech sound integration. Interestingly, it has been reported that subjects' percept of the number of flashes can be strongly influenced by the number of concurrent beeps, indicating a strong auditory influence on vision (Andersen, Tiippana, \& Sams, 2004). Remarkably, in contrast with for example the McGurk illusion in which vision dominates audition (McGurk \& MacDonald, 1976), the flashes evoked no illusory change in the percept of the number of beeps when the task was to focus on the beeps. Again, this study used arbitrarily linked stimuli, supporting our suggestion that the nature of the link between the two modalities of a stimulus may be critical for the automaticity of the involvement of both low level sensory cortices. And again, the task employed was found to be critical, since in another condition in which the task required to focus on both the beeps and flashes illusions were reported in both modalities.

The present findings, together with previous findings from our group, address the question posed by Naumer and colleagues in a study on the neural network involved in processing object familiarity and semantic congruency (Hein et al., 2007). The authors wandered in the conclusions on $\mathrm{p} 7886$ "....whether even sensory specific regions become involved in audiovisual integration of artificial object features if the respective associations are explicitly trained". While low level visual processing seems not to be automatically involved during integration of artificially linked audiovisual stimuli (present study; Van Atteveldt, Formisano, Goebel, \& Blomert, 2004; Van Atteveldt, Formisano, Blomert, \& Goebel, 2007), low level auditory processing is (Blau, van Atteveldt, Formisano, Goebel, \& Blomert, 2008; Froyen, van Atteveldt, Bonte, \& Blomert, 2008; Van Atteveldt, Formisano, Blomert, \& Goebel, 2007; Van Atteveldt, Formisano, Goebel, \& Blomert, 2004), but requires multiple years of explicit training (Froyen, Bonte, van Atteveldt, \& Blomert, 2009).

Moreover, the presently described difference in the involvement of low level visual areas during letter - speech sound processing is interesting for further exploration in the light of normal and abnormal reading development. In developmental dyslexia, for example, it is remarkable how specific reading and writing skills are defi- 
cient while other skills are largely unaffected (Vellutino, Fletcher, Snowling, \& Scanlon, 2004). The uniqueness of written language, considering its phylogenetic and ontogenetic development as well as its arbitrary link with phonology, might contribute to the specificity of the reading and writing deficit in people with dyslexia.

The auditory MMN has been shown to be a valuable tool to investigate, noninvasively and without the requirement of a task, auditory and phonological processing (Bishop, 2007; Bonte, Poelmans, \& Blomert, 2007; Csépe, 2003; Kujala \& Naatanen, 2001) as well as letter - speech sound processing (Froyen, Bonte, van Atteveldt, \& Blomert, 2009; Froyen, Willems, \& Blomert, submitted) in normal and reading impaired children. For the same reasons, the visual MMN might contribute to investigations of visual or crossmodal processing in normal or impaired reading adults or children.

In closing it should be noted that, while there was no direct effect of speech sounds on letter processing, the speech sounds suppressed non-letter processing. One reason might be that, because the experimental stimulus context existed almost entirely of letter - speech sound pairs, an attention set was build up in which nonletter processing in this context was suppressed as distracting. In another audiovisual MMN-study with meaningless stimuli, the visual deviant in the audiovisual experiment was also found to evoke a lower visual MMN amplitude in comparison with that same visual deviant in the visual only experiment (Besle, Fort, \& Giard, 2005). Although a content-related feedback mechanism is not a likely explanation for this effect, considering their use of meaningless stimuli, it at least implies that such audiovisual interactions can be measured with the vMMN. This re-assures that the visual $\mathrm{MMN}$ is an appropriate tool to investigate the involvement of lower level visual processing during passive audiovisual integration.

Independent of the exact nature of the suppression mechanism, our findings imply that whereas low level auditory processing seems to constitute a standard ingredient in letter - speech sound processing (Froyen, Bonte, van Atteveldt, \& Blomert, 2009; Froyen, van Atteveldt, Bonte, \& Blomert, 2008; Van Atteveldt, Formisano, Blomert, \& Goebel, 2007; Van Atteveldt, Formisano, Goebel, \& Blomert, 2004), this does not hold for low level visual processing. This does not mean that speech sounds cannot influence letter processing indirectly as evidenced in the present suppression effects for non-letter processing, but emphasizes that speech sound processing does not automatically impact on letter processing and therefore does not seem to constitute an integral part of the letter - speech sound integration network. 


\section{Conclusion}

Although previous research revealed systematic influences of letters on speech sound processing, we did not find the reverse pattern; i.e., influences of speech sounds on letter processing in a cross-modal context. The results reveal that in contrast to well characterized natural audiovisual association processes like audiovisual speech processing, the network for letter - speech sound integration is characterized by an asymmetric role of low level sensory areas; auditory areas are automatically recruited whereas low level visual processing is not directly involved. Only nonletter processing was suppressed, when simultaneously presented with speech sounds, possibly pointing to regulatory feedback from audiovisual integration sites to visual cortex. The fact that written language is not naturally related to speech, may account for the finding that only low level auditory, but not low level visual cortex is involved in the automatic integration of letters and speech sounds, a necessary prerequisite for developing reading fluency.

\section{Acknowledgements}

This work was supported by grant 608/002/2005 of the Dutch Board of Health Care Insurance (College voor Zorgverzekeringen) awarded to L.B. We thank Hanne Poelmans for assistance in data acquisition.

\section{References}

Ahlo, K. (1995). Cerebral Generators of mismatch negativity (MMN) and its magnetic counterpart (MMNm) elicited by sound changes. Ear \& Hearing, 16(1), 38-51.

Alho, K., Woods, D., Algazi, A., \& Naatanen, R. (1992). Intermodal selective attention. II. Effects of attentional load on processing of auditory and visual stimuli in central space. Electroencephalography and clinical Neurophysiology, 82, 356-368.

Amedi, A., von Kriegstein, K., Van Atteveldt, N. M., Beauchamp, M. S., \& Naumer, M. J. (2005). Functional imaging of human crossmodal identification and object recognition. Experimental Brain Research, 166, 559-571.

Andersen, T. S., Tiippana, K., \& Sams, M. (2004). Factors influencing audiovisual fission and fusion illusions. Cognitive Brain Research, 21(3), 301-308.

Astikainen, P., Ruusuvirta, T., Wikgen, J., \& Korjonen, T. (2007). Visual mismatch negativity. Journal of psychophysiology, 21, 224-230.

Berti, S., \& Schröger, E. (2003). Distraction effects in vision: Behavioral and event-related potential effects. Neuroreport, 15, 665-669.

Besle, J., Fort, A., \& Giard, M. (2005). Is the auditory memory sensitive to visual information. Experimental Brain Research, 166, 337-344.

Bishop, D. V. M. (2007). Using mismatch negativity to study central auditory processing in developmental language and literacy impairments: Where are we, and where should we be going? Psychological bulletin, 133, 651-672.

Blau, V., Atteveldt, N., Ekkebus, M., Goebel, R., \& Blomert, L. (2009). Reduced neural integration of letters and speech sounds links phonological and reading deficits in adult dyslexia. Current Biology, $19,503-508$. 
Blau, V., van Atteveldt, N., Formisano, E., Goebel, R., \& Blomert, L. (2008). Task-irrelevant visual letters interact with the processing of speech sounds in heteromodal and unimodal cortex. European journal of neuroscience, 28(3), 500-509.

Boersma, P., \& Weenink, D. (2002). Praat 4.0: a system for doing phonetics with the computer. Amsterdam: Universiteit van Amsterdam.

Bonte, M., Poelmans, H., \& Blomert, L. (2007). Deviant neurophysiological responses to phonological regularities in speech in dyslexic children. Neuropsychologia, 45, 1427-1437.

Burnham, D., \& Dodd, B. (2004). Auditory - visual speech integration by prelinguistic infants: Perception of an emergent consonant in the McGurk effect. developmental psychobiology, 45, 204 - 220.

Calvert, G. A. (2001). Crossmodal processing in the human brain: insights from functional neuroimaging studies. Cereb Cortex, 11(12), 1110-1123.

Calvert, G. A., Brammer, M. J., Bullmore, E. T., Campbell, R., Iversen, S. D., \& David, A. S. (1999). Response amplification in sensory-specific cortices during crossmodal binding. Neuroreport, 10(12), 26192623.

Calvert, G. A., Brammer, M. J., \& Iversen, S. D. (1998). Crossmodal identification. Trends in Cognitive Sciences, 2, 247-253.

Calvert, G. A., Campbell, R., \& Brammer, M. J. (2000). Evidence from functional magnetic resonance imaging of crossmodal binding in the human heteromodal cortex. Current Biology, 10(11), 649-657.

Colin, C., Radeau, M., Soquet, A., Demolin, D., Colin, F., \& Deltenre, P. (2002). Mismatch negativity evoked by the McGurk-MacDonald effect: a phonetic representation within short-term memory. Clin Neurophysiol, 113(4), 495-506.

Csépe, V. (2003). Dyslexia: different Brain, Different Behavior. New York: Kluwer Academic/ Plenum Publishers.

Czigler, I. (2007). Visual mismatch negativity; Violation of nonattended environmental regularities. Journal of Psychophysiology, 21, 224-230.

Czigler, I., Balazs, L., \& Pato, L. (2004). Visual change detection: event-related potentials are dependent on stimulus location in humans. Neuroscience letters, 364, 149-153.

de Gelder, B., Bocker, K. B., Tuomainen, J., Hensen, M., \& Vroomen, J. (1999). The combined perception of emotion from voice and face: early interaction revealed by human electric brain responses. Neuroscience Letters, 260(2), 133-136.

Dijkstra, T., Frauenfelder, U. H., \& Schreuder, R. (1993). Bidirectional grapheme-phoneme activation in a bimodal detection task. Journal of Experimental Psychology: Human Perception and Performance, 19(5), 931-950.

Dijkstra, T., Schreuder, R., \& Frauenfelder, U. H. (1989). Grapheme Context Effects on Phonemic Processing. Language and Speech, 32, 89-108.

Ehri, L. C. (2005). Development of sight word reading: phases and findings. In M. J. Snowling \& C. Hulme (Eds.), The science of reading: A handbook (pp. 135 - 145). Oxford: Blackwell Publishing.

Fort, A., \& Giard, M. (2004). Multiple electrophysiological mechanisms of audiovisual integration in human preception. In G. A. Calvert, C. Spence \& B. E. Stein (Eds.), The handbook of multisensory processes (pp. 503 - 513). London: The Mitt Press.

Froyen, D., Bonte, M., van Atteveldt, N., \& Blomert, L. (2009). The long road to automation: Neurocognitive development of letter-speech sound processing. Journal of Cognitive Neuroscience, 21, 567 580.

Froyen, D., van Atteveldt, N., Bonte, M., \& Blomert, L. (2008). Cross-modal enhancement of the MMN to speech sounds indicates early and automatic integration of letters and speech sounds. Neuroscience Letters, 430, 23-28.

Froyen, D., Willems, G., \& Blomert, L. (submitted). Evidence for a specific cross-modal binding deficit in dyslexia: An MMN-study of letter - speech sound processing.

Giard, M. H., Perrin, F, Pernier J, Bouchet P. (1990). Brain generators implicated in the processing of auditory stimulus deviance: a topographic event-related potential study. Psychophysiology, 27, 627640. 
Hardy, M. H., Smythe, P. C., Stennet, R. G., \& Wilson, H. R. (1972). Developmental patterns in elemental reading skills: phoneme-grapheme and grapheme-phoneme correspondences. Journal of Educational Psychology, 63, 433-436.

Hashimoto, R., \& Sakai, K. L. (2004). Learning letters in adulthood: direct visualization of cortical plasticity for forming a new link between orthography and phonology. Neuron, 42(2), 311-322.

Hein, G., Doehrmann, O., Müller, N. G., Kaiser, J., Muckli, L., \& Naumer, M. J. (2007). Object familiarity and semantic congruency modulate responses in cortical audiovisual integration areas. The Journal of Neuroscience, 27, 7881-7887.

Herdman, A. T., Fujioka, T., Chau, W., Ross, B., Pantev, C., \& Picton, T. W. (2006). Cortical oscillations related to processing congruent and incongruent grapheme-phoneme pairs. Neuroscience Letters, 399, $61-66$.

Heslenfeld, D. J. (2003). Visual mismatch negativity. In J. Polich (Ed.), Detection of change: Event related potential and fMRI findings. Boston: Kluver Academic Press.

Kenemans, J. L., Grent-'t Jong, T., \& Verbaten, M. N. (2003). Detection of visual change: Mismatch or rareness? NeuroReport, 14, 1239-1242.

Kimura, M., Katayama, J., Ohira, H., \& Schröger, E. (2009). visual mismatch negativity: New evidence from the equiprobable paradigm. Psychophysiology, 46, 402-409.

Kujala, T., \& Naatanen, R. (2001). The mismatch negativity in evaluating central auditory dysfunction in dyslexia. Neurosci Biobehav Rev, 25(6), 535-543.

Macaluso, E., George, N., Dolan, R., Spence, C., \& Driver, J. (2004). Spatial and temporal factors during processing of audiovisual speech: a PET study. Neurolmage, 21, 725-732.

Maekawa, T., Goto, Y., Kinukawa, N., Taniwaki, T., Kanba, S., \& Tobimatsu, S. (2005). Functional characterization of mismatch negativity to a visual stimulus. Clinical neurophysiology, 116, 2392-2402.

McCandliss, B. D., \& Noble, K. G. (2003). The development of reading impairment: a cognitive neuroscience model. Ment Retard Dev Disabil Res Rev, 9(3), 196-204.

McGurk, H., \& MacDonald, J. (1976). Hearing lips and seeing voices. nature, 263, 747.

Möttönen, R., Krause, C. M., Tiippana, K., \& Sams, M. (2002). Processing of changes in visual speech in the human auditory cortex. Brain Res Cogn Brain Res, 13(3), 417-425.

Munhall, K., \& Vatikiotis-Bateson, E. (1998). The moving face during speech communication. In R. Campbell, B. Dodd \& D. Burnham (Eds.), Hearing by Eye, Part 2: The psychology of speechreading and audiovisual speech (pp. 123-139). London, UK: Taylor \& Francis, Psychology Press.

Näätänen, R. (1995). The Mismatch Negativity: A powerful tool for cognitive neuroscience. Ear and Hearing, 16, 6-18.

Pazo-Alvarez, P., Cadaveira, F., \& Amenedo, E. (2003). MMN in the visual modality: a review. Biol Psychol, 63(3), 199-236.

Raij, T., Uutela, K., \& Hari, R. (2000). Audiovisual integration of letters in the human brain. Neuron, 28, 617-625.

Sams, M., Aulanko, R., Hamalainen, M., Hari, R., Lounasmaa, O. V., Lu, S. T., et al. (1991). Seeing speech: visual information from lip movements modifies activity in the human auditory cortex. Neurosci Lett, 127(1), 141-145.

Schröger, E. (1998). Measurement and interpretation of the mismatch negativity. Behavior Research Methods Instruments \& Computers, 30, 131-145.

Stekelenburg, J. J., Vroomen, J., \& de Gelder, B. (2004). Illusory sound shifts induced by the ventriloquist illusion evoke the mismatch negativity. Neuroscience Letters, 357, 163-166.

Tales, A., \& Butler, S. (2006). Visual mismatch negativity highlights abnormal preattentive visual processing in Alzheimer's disease. Neuroreport, 17, 887-890.

Tales, A., Haworth, J., Wilcock, G., Newton, P., \& Butler, S. (2008). Visual mismatch negativity highlights abnormal pre-attentive visual processing in mild cognitive impairment and Alzheimer's disease. Neuropsychologia, 46, 1224-1232.

Tales, A., Newton, P., Troscianko, T., \& Butler, S. (1999). Mismatch negativity in the visual modality. Neuroreport, 10(16), 3363-3367. 
Talsma, D., Doty, T. J., \& Woldorff, M. G. (2007). Selective attention and audiovisual integration: Is attenting to both modalities a prerequisite for early integration. Cerebral Cortex, 17, 679-690.

Van Atteveldt, N., Formisano, E., Blomert, L., \& Goebel, R. (2007). The effect of temporal asynchrony on the multisensory integration of letters and speech sounds. Cerebral Cortex, 13, 962-974.

Van Atteveldt, N., Formisano, E., Goebel, R., \& Blomert, L. (2004). Integration of letters and speech sounds in the human brain. Neuron, 43, 271-282.

Van Atteveldt, N., Formisano, E., Goebel, R., \& Blomert, L. (2007). Top-down task effects overrule automatic multisensory responses to letter-sound pairs in auditory association cortex. Neurolmage, 36, 1345-1360.

Vellutino, F. R., Fletcher, J. M., Snowling, M. J., \& Scanlon, D. M. (2004). Specific reading disability (dyslexia): what have we learned in the past four decades? J Child Psychol Psychiatry, 45(1), 2-40.

Wei, J. H., Chan, T. C., \& Luo, Y. J. (2002). A modified oddball paradigm "cross-modal delayed response" and the research on mismatch negativity. Brain Res Bull, 57(2), 221-230. 
Chapter 6

\section{Letter-specificity of previously reported cross-modal effects on MMN to speech sounds}

Froyen, D., de Doelder, N., \& Blomert, L. (in preparation). Letter-specificity of previously reported crossmodal effects on MMN to speech sounds 


\begin{abstract}
Recently, a cross-modal MMN paradigm has been proposed to measure, preattentively and with high temporal resolution, neural correlates of letter - speech sound processing. When letters were presented simultaneously with speech sounds, MMN amplitude to speech sounds was found to be enhanced in comparison with the MMN to speech sounds presented in isolation, indicating early and automatic letter - speech sound integration. Although there were strong indications for the letter - specificity of the previously reported effects, alternative explanations related to the difference in context, auditory only versus audiovisual stimulus presentation, cannot be excluded.

In the present study we carefully controlled for differences in visual stimulation by including an audiovisual non-letter condition besides an audiovisual letter condition. Furthermore, different letters and speech sounds were chosen to allow generalization of the previous results. The present results revealed a clear enhancement of MMN amplitude when speech sounds were presented with letters as well as nonletters. Additionally, MMN amplitude in the letter condition differed significantly from MMN amplitude in the non-letter condition. Remarkably, considering that the participants were all fluently reading adults without any history of reading problems, a correlation was found between the letter-specific MMN enhancement effect and reading fluency.

These results clearly indicate that the previously reported letter - speech sound integration effect was genuine, can be generalized to other letter - speech sound pairs and, moreover, that it is strongly related with reading fluency.
\end{abstract}




\section{Introduction}

In the present society, fluent reading and writing skills are crucial to obtain social and economic success. Most models describing the process of learning to read emphasize the acquisition of the association of letters with their corresponding speech sounds as a basic requirement for learning to read (Ehri, 2005; Frith, 1985). Recently, the interest in letter - speech sound processing has increased dramatically. Several functional magnetic resonance imaging ( $f M R I)$ studies have found corroborating results indicating multisensory integration areas in the temporal lobes as well as low level auditory areas to be involved during letter - speech sound integration (Blau, van Atteveldt, Formisano, Goebel, \& Blomert, 2008; Hashimoto \& Sakai, 2004; Van Atteveldt, Formisano, Blomert, \& Goebel, 2007; Van Atteveldt, Formisano, Goebel, \& Blomert, 2004; Van Atteveldt, Formisano, Goebel, \& Blomert, 2007). Two magnetoencephalography (MEG) studies confirmed the involvement of these areas and provided high temporal resolution information, indicating letter - speech sound integration effects within 250 to 300 ms after stimulus onset (Herdman et al., 2006; Raij, Uutela, \& Hari, 2000). However, these MEG studies used an active task to ensure participants constant focus on the stimuli, i.e., subjects had to judge the congruency of the letter - speech sound pair presented. In a recent fMRI study, it was shown that such an active congruency judgment task overruled the congruency effects found in previous studies using a passive design. This task effect was interpreted as a consequence of the fact that congruent as well as incongruent stimuli became equally relevant given the task. (Van Atteveldt, Formisano, Goebel et al., 2007). This study indicated that the neural network involved in active letter - speech sound processing, might differ from passive/automatic letter - speech sound processing.

Recently, a cross-modal MMN paradigm was proposed to investigate neural correlates of letter - speech sound processing with a high temporal resolution method, but without an attention demanding task (Froyen, van Atteveldt, Bonte, \& Blomert, 2008). The MMN is known to reflect an automatic and purely auditory deviance detection mechanism (Alho, 1995; Giard, 1990). It is evoked between 100 and 200 ms after stimulus onset when in a sequence of auditory stimuli a rarely presented sound (the deviant) deviates in one or more aspects from a frequently presented sound (the standard) (Näätänen, 1995; Schröger, 1998). In Froyen and coauthors (2008), the MMN evoked by a deviant speech sound violating a standard speech sound (auditory only condition) was compared with the MMN evoked by the same deviant speech sound but violating both the standard speech sound and the simultaneously presented standard letter (audiovisual condition). The MMN amplitude evoked in the audiovisual condition was found to be enhanced in comparison with the MMN amplitude in the auditory only condition. Given the early and automatic evocation of the $M M N$, this enhancement was interpreted to reflect early and automatic letter - speech sound integration in experienced adult readers. It was 
suggested that the enhancement was caused by the double, cross-modal deviation of the deviant speech sound from the standard speech sound and the standard letter. Later, the exact same paradigm was successfully employed to investigate letter - speech sound processing in typically reading children (Froyen, Bonte, van Atteveldt, \& Blomert, 2009) as well as in children with developmental dyslexia (Froyen, Willems, \& Blomert, submitted).

All MMN studies revealed robust effects of letters on speech sound processing, thus indicating that the observed modulation of the MMN very likely was letter-specific. It can however not been ruled out that these effects occurred as a consequence of other task or stimulus related aspects. In the previous fMRI studies employing a passive 'task' design it was shown that the congruency of the letter - speech sound pairs systematically modulated the brain response to speech sounds: i.e., when the pair was congruent the response was enhanced, when the pair was incongruent the response was suppressed (e.g. Van Atteveldt et al, 2004). The congruency manipulation thus ensured the validity of the interpretation of the observed effects as letterspecific. In contrast to such a design, the MMN paradigm does not allow a comparable congruency manipulation. Indications for the effects of letters on speech sounds are based on the observation that the amplitude of the mismatch to speech sounds is modulated by the also presented deviant letter. The crucial evidence in this design thus consists of the comparison of the $\mathrm{MMN}$ in a unimodal auditory only condition with the MMN in a cross-modal audiovisual condition. Although the crossmodal MMN paradigm appeared to be suitable for the investigation of letter speech sound integration (Froyen et al., 2009; Froyen et al., 2008), it cannot be excluded that the MMN amplitude was affected by the presence of a visual stimulus as such. While in the auditory only condition participants were watching a silent movie, in the audiovisual condition letters were simultaneously presented with speech sounds. Furthermore, to ensure that the subjects were focusing the screen in the cross-modal condition, subjects had to press a button whenever a colored picture of a present appeared on the screen. It is possible that the unrelated task or the difference in visual stimulation had an unspecific effect on the MMN to speech sounds. An argument against such an explanation was the linear effect of stimulus onset asynchrony (SOA) observed in the adult study (Froyen et al., 2008). To investigate the time window of integration, in the audiovisual condition the letter was presented either simultaneously or 100 or $200 \mathrm{~ms}$ before the speech sound. Although the task was the same for all three SOA conditions, we observed a linear decrease in MMN amplitude with increasing SOA. At 200 ms SOA, the MMN did not differ in amplitude, or in any other aspect, from the MMN observed in the auditory only condition in which no task was required. We furthermore checked the brain waves in the audiovisual experiments before the onset of the auditory stimulus, to control for possible contaminations of the auditory evoked potentials by visual evoked onset potentials. The presented letter was identical in the standard and 
deviant conditions and accordingly elicited comparable visual evoked responses, resulting in the absence of a difference potential prior to auditory stimulus onset at SOA 100 and 200 ms. This similarity in evoked responses in the standard and the deviant condition indicated the absence of a general modulation by overlapping visual and auditory evoked potentials at frontocentral electrodes at which the MMN was measured. Although these observations underline our interpretation of the MMN amplitude enhancement as letter-specific, a more direct investigation of the letter-specificity of the MMN effects seems warranted.

\section{The present study}

In the present study we aimed at explicitly investigating the letter-specificity of the previously found cross-modal amplitude enhancement of the MMN to speech sounds. Speech sounds were presented either in isolation or simultaneously with letters or non-letters, which were a scrambled version of the letters. Since in the previous studies always the speech sounds /a/ and /o/ were used (Froyen et al., 2009; Froyen et al., 2008), we now used as a standard the speech sound /e/ and as a deviant the speech sound $/ \mathrm{u} /$ in order to be able to generalize the previously reported effect to other letter - speech sound pairs. Furthermore, since the previous MMN studies employed a between subject design we now presented all conditions within subject to ensure optimal comparability of the conditions. This also made it possible to calculate a letter effect measure for each subject and to test for correlations of this letter effect measure with behavioral reading measures.

We expect first an enhancement of the MMN to speech sounds whenever a letter is presented with the speech sound and thus a replication of our earlier results with different stimuli. Furthermore, if this MMN modulation effect is letter-specific, we do not expect a comparable modulation if the non-letter is presented.

\section{Methods}

\section{Participants}

Twenty-one subjects participated in the present experiment ( 14 female, range 19 to 33 , mean age 23 years and 4 months). Subjects gave informed written consent and were paid for their participation. All subjects were undergraduate university students without any history of hearing or reading problems and with normal or corrected to normal vision. Two data sets were discarded due to excessive head movements. 


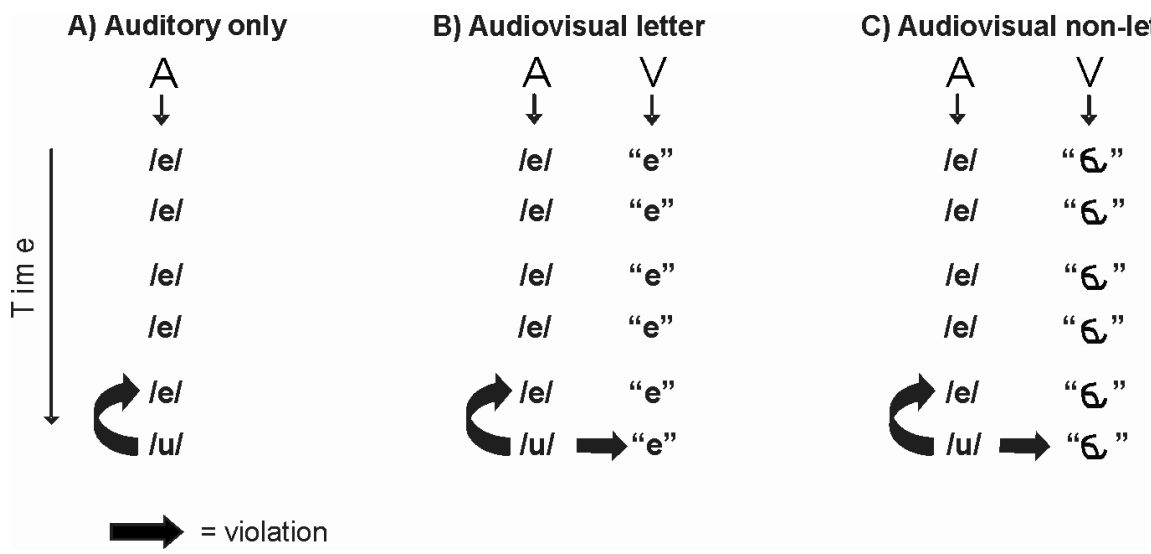

Figure 1. Designs of the auditory only (A), the audiovisual letter (B) and the audiovisual non-letter (C) condition. " $A$ " represents auditory stimulus presentation, " $V$ " represents visual stimulus presentation. The arrow indicates the violation of the standard speech sound in the auditory only condition $(A)$ and the double cross-modal violation in the audiovisual conditions ( $\mathrm{B}$ and $\mathrm{C}$ ).

\section{Stimuli}

Stimuli were natural speech sounds, /e/ and / $\mathrm{u} /$, the visually presented single letter "e", and the visually presented non-letter " $\sigma$ ". speech sounds were digitally recorded (sampling rate $44.1 \mathrm{kHz}, 16$ bit quantization) from a female speaker. Recordings were band-pass filtered (180-10.000 Hz), resampled at $22.05 \mathrm{kHz}$ and matched for loudness with Praat software (Boersma \& Weenink, 2002). The sounds were presented binaurally through loudspeakers at about $65 \mathrm{~dB}$ SPL and had a duration of $300 \mathrm{~ms}$. The visual stimuli were presented in white on a black background in the centre of a computer screen for $500 \mathrm{~ms}$, printed in lower case font "Arial" at letter size 40 . The non-letter was a scrambled version of the letter "e". Separate psychophysical tests were performed before the execution of the present experiment in order to choose the optimal scrambled letter out of eight different scrambled versions of " $\mathrm{e}$ " and " $\mathrm{u}$ ". Between the presentations of the stimuli a white fixation cross was presented in the centre of the screen.

\section{Procedure}

Each condition consisted of 12 experimental blocks with 400 trials. Trial length was always $1250 \mathrm{~ms}$. There were three types of blocks: The auditory blocks (A) in which participants were watching a silent movie while the speech sounds /e/ (90\%) and $/ \mathrm{u} /(10 \%)$ were presented, or one of the two audiovisual blocks in which simultaneously with the speech sounds /e/ (90\%) and /u/ (10\%) either the letter " $\mathrm{e}$ " (AVL) or the non-letter " 6 " (AVNL) was presented. To ensure that subjects were at all times focusing the screen during the audiovisual blocks, we interspersed the experimental trials with a non-related color picture. Subjects had to press a button when they saw 
the picture. Trials within the blocks were randomly presented. Blocks were pseudo randomly presented.

\section{Recording}

EEG data were recorded with NeuroScan 4.2 from 0.01 to $50 \mathrm{~Hz}$ with a sampling rate of $250 \mathrm{~Hz}$ in a sound-attenuating and electrically shielded room from 30 electrode positions (Extended International 10-20 system) relative to a nose reference. Eye-movements and blinks were measured with bipolar VEOG/HEOG channels. All electrode impedance levels (EEG and EOG) were kept below $5 \mathrm{k} \Omega$. EEG data were band pass filtered $(1-30 \mathrm{~Hz})$ with NeuroScan 4.2, epoched from -100 to $1000 \mathrm{~ms}$ relative to stimulus onset and baseline corrected (100 ms pre-stimulus interval). Epochs containing data exceeding a maximum voltage criterion of $75 \mu \mathrm{V}$ were rejected. Standards immediately following deviants were not included in the analyses, as were the trials with target pictures. The raw EEG data were corrected for vertical eye-movements (i.e. blink artifacts). In the auditory condition we included 176 (88\%) of the standard trials and $173(86.5 \%)$ of the deviant trials. In AVL we retained respectively 174 (87\%) and 178 (89\%), and in AVNL 178 (89\%) and 180 (90\%) of the trials. Epochs were averaged separately for each condition and each participant from the onset of the auditory stimulus. Difference waves were calculated by subtracting the ERPs to the standard speech sound from ERPs to the deviant speech sound.

\section{Statistical analysis}

Amplitude measures were obtained by taking the mean amplitude of the grand average and difference waves from the $50 \mathrm{~ms}$ time window around the individual peak latency. The individual peak latency was determined separately for each subject, condition and electrode site between 50 and $250 \mathrm{~ms}$ after stimulus onset, the time-window typically reported in MMN-studies (Schröger, 1998).

First the mean area amplitudes of the grand average waves in the three conditions were investigated to assure differences between standards and deviants reflecting a MMN-like response to deviants. Statistical analyses were performed first on the grand average waves of eleven electrode sites covering the fronto-central ( $\mathrm{Fz}, \mathrm{Cz}$, FC3 and FC4), temporal (T3 and T4), parietal (CP3, CP4), and occipital (O1, O2 and $\mathrm{Oz}$ ) regions of the brain. When there was an interaction with electrode sites, subsequent analyses were performed on the four frontocentral electrodes ( $F z, C z, F C 3$ and FC4) where the MMN effect was most prominent.

Subsequently, difference waves' amplitudes were analyzed with a repeated measures ANOVA with the frontocentral electrodes ( $\mathrm{Fz}, \mathrm{Cz}, \mathrm{FC} 3$ and $\mathrm{FC} 4$ ) and condition (A, AVL and AVNL) as within subject factors to check for an effect of the visual stimuli on MMN amplitude and latency. All results are Greenhouse-Geiser corrected. 
Because we had a-priori expectations about the direction of the differences in MMN amplitude between the conditions, we calculated contrasts to analyze these differences in detail.

Finally, since we collected behavioral reading scores to ensure that all subjects have proficient reading skills, it was possible to check for correlations with reading fluency and an individually calculated letter - specific MMN enhancement measure. In a previous study of letter - speech sound processing in dyslexic children, a strong correlation was found with word reading fluency and a somewhat lower correlation with non-word reading fluency (Froyen et al., 2009). Since we have strong predictions about the direction of this correlation, namely a stronger effect of letters on speech sound processing in subjects with better word and non-word reading fluency, a one-tailed correlation was performed.

\section{Results}

\section{Typical MMN in all conditions}

In all three conditions ( $\mathrm{A}, \mathrm{AVL}$ and $\mathrm{AVNL}$ ) the deviant speech sound stimulus elicited a negativity around $200 \mathrm{~ms}$ after auditory stimulus onset with a typical topographical distribution of the MMN (Figures 2 and 3). Average peak latencies (averaged over the four frontocentral electrodes) were $177 \mathrm{~ms}$ (range: 167 - $187 \mathrm{~ms}$ ) for $\mathrm{A}$, $202 \mathrm{~ms}$ (range: 195 - $210 \mathrm{~ms}$ ) for AVL, and $206 \mathrm{~ms}$ (range: 199 - $212 \mathrm{~ms}$ ) for AVNL after the onset of the auditory stimulus deviation.

A repeated measures ANOVA was performed with three within subject factors; stimulus (standard versus deviant), condition ( $A, A V L$ and $A V N L)$ and electrode sites ( $F z, C z, F C 3, F C 4, T 3, T 4, C P 3, C P 4, O 1, O 2$ and Oz). There was a trend towards a three-way interaction, $F(20,360)=2.4, p=0.083$. Since we expected an interaction due to differential effects of stimulus type depending on the electrode measured, a subsequent analysis was performed on only the four fronto-central electrodes ( $F z$, $\mathrm{Cz}, \mathrm{FC} 3$ and FC4) were the MMN is expected to be most pronounced. A repeated measures analysis with stimulus, condition and only the four fronto-central electrode sites revealed no three way interaction, $F(6,108)=1.5, p=0.199$, no twoway interaction between condition and electrode sites, $F(6,108)=1.5, p=0.220$ and no two-way interaction between stimulus and electrode sites, $F(3,54)=1.6, p$ $=0.196$. Interestingly, there was a significant interaction between stimulus and condition, $F(2,36)=13.6, p<.001$, indicating a different effect of speech sound deviants depending on the condition. 


\section{A. auditory only}
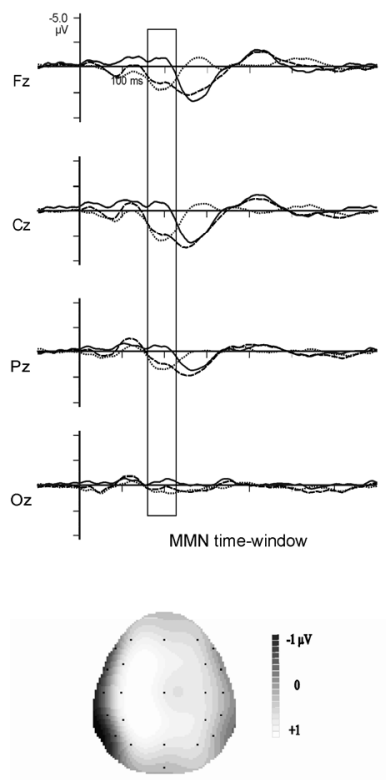

$180 \mathrm{~ms}$
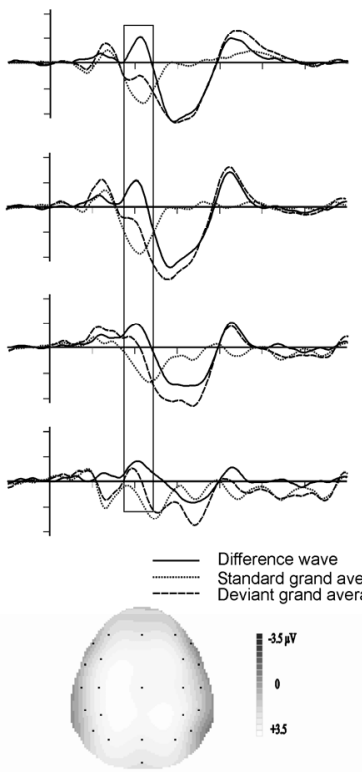

$200 \mathrm{~ms}$
C. audiovisual non-letter

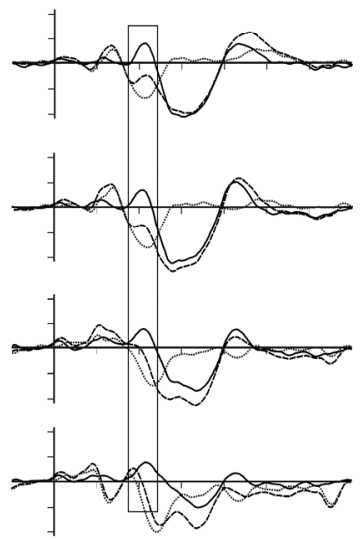

Figure 2. Grand average waves to the standard and deviant sounds and difference waves as observed on the midline electrodes $(\mathrm{Fz}, \mathrm{Cz}, \mathrm{Pz}$ and $\mathrm{Oz})$ in the auditory only condition $(\mathrm{A})$, the audiovisual letter condition (B) and the audiovisual non-letter condition (C). The MMN time window is indicated with a rectangle. Below, topographical maps at the peak of the difference wave in the MMN time window.

A repeated measures ANOVA with stimulus (standard versus deviant) and electrode sites ( $F z, C z, F C 3$ and FC4) performed per condition separately revealed no two-way interaction and a significant main effect of stimulus in each condition; $F(3,54)=1.8$, $p=.158$ and $F(1,18)=10.4, p=.005$, for the auditory only condition $F(3,54)=2.1$, $p=.117$ and $F(1,18)=29.3, p<.001$, for the audiovisual letter condition, and, $F(3$, $54)<1$ and $F(1,18)=4.6, p=.045$, for the audiovisual non-letter condition. The speech sound deviant evoked more negative grand average wave amplitudes in comparison with the standard speech sound in each condition separately, indicating a typical MMN in all conditions. 


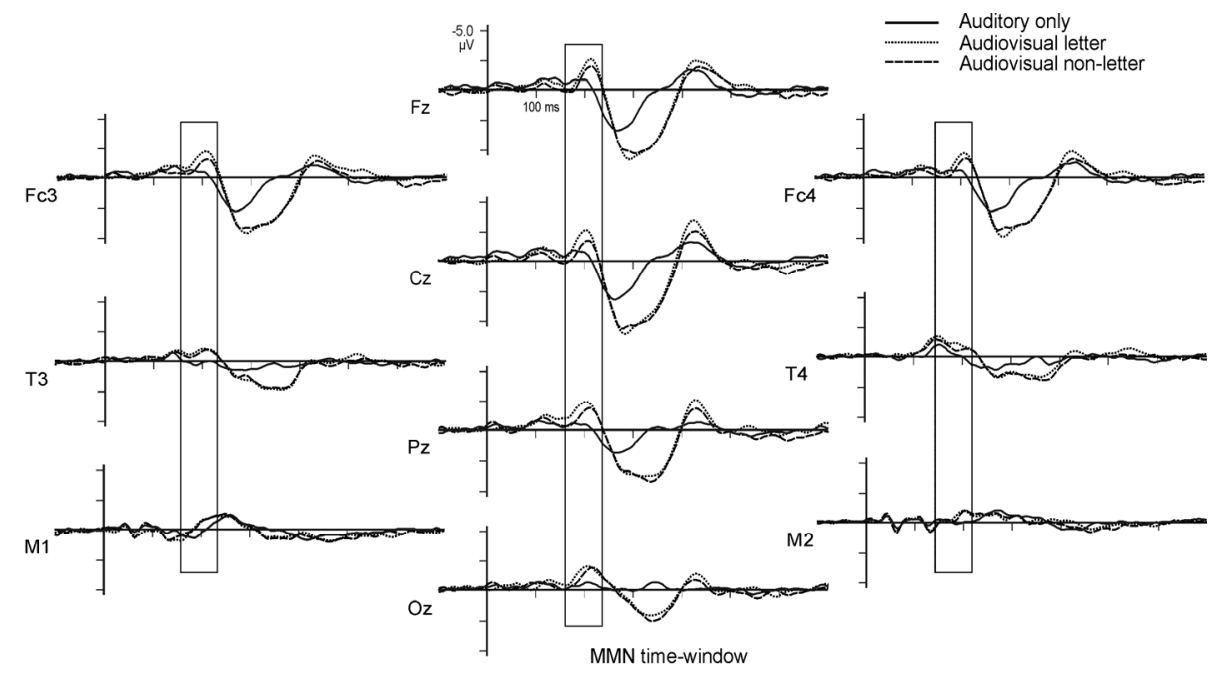

Figure 3. Difference waves as observed on $\mathrm{Fz}, \mathrm{Cz}, \mathrm{Pz}, \mathrm{Oz}, \mathrm{Fc} 3, \mathrm{Fc} 4, \mathrm{~T} 3, \mathrm{~T} 4, \mathrm{M} 1$ and $\mathrm{M} 2$ in the auditory only condition (solid line), the audiovisual letter condition (dotted line) and the audiovisual non-letter condition (dashed line). The MMN time window is indicated with a rectangle.

\section{Effect of visual stimulus on $M M N$ to speech sounds}

In order to check for an effect of letters on early speech sound processing, the MMN evoked in the auditory condition was compared with the MMN in both audiovisual conditions ( $A V L$ and $A V N L)$. The mean area amplitudes of the difference waves were analyzed with a repeated measures ANOVA with condition ( $A, A V L$ and $\mathrm{AVNL}$ ) and electrode sites ( $\mathrm{Fz}, \mathrm{Cz}, \mathrm{FC} 3$ and $\mathrm{FC} 4)$ as within subject factors. There was a significant main effect of condition, $F(2,36)=9.3, p=.001$. Predefined contrasts revealed a significant difference considering the difference wave amplitude between A and AVL, $t(18)=3.9, p=.001$, but also between A and AVNL, $t(18)=2.4, p$ $=.027$, and between AVL and AVNL, $t(18)=-2.1, p=.048$. This indicates a general effect of the visual stimulus on speech sound processing, but additionally a letterspecific effect when this visual stimulus is a letter as compared to a scrambled letter.

\section{Correlation with reading fluency}

Single measures of the letter and the non-letter effect on MMN amplitude were calculated per individual to allow checking for correlations with reading fluency. The amplitude of the MMN in the audiovisual letter condition was subtracted from the amplitude of the $\mathrm{MMN}$ in the audiovisual non-letter condition, to control for nonspecific audiovisual effects and thus obtain a letter effect measure reflecting purely the letter-specific effect on speech sound processing. A correlation was found between the letter effect measure and word reading as measured by a word reading 
test (Brus \& Voeten, 1973), $r=-.421 ; p=.041$, and by a computerized word reading test (Blomert \& Vaessen, 2009), $r=-.400 ; p=.050$ (Figure 4). A slightly lower correlation was found with non-word reading (KLEPEL), $r=-.254 ; p=.155$. Lower MMN amplitude means stronger effect of letter on speech sound processing. Consequently, a negative correlation with reading performance indicates that a high reading level coincides with stronger letter - speech sound integration effects.
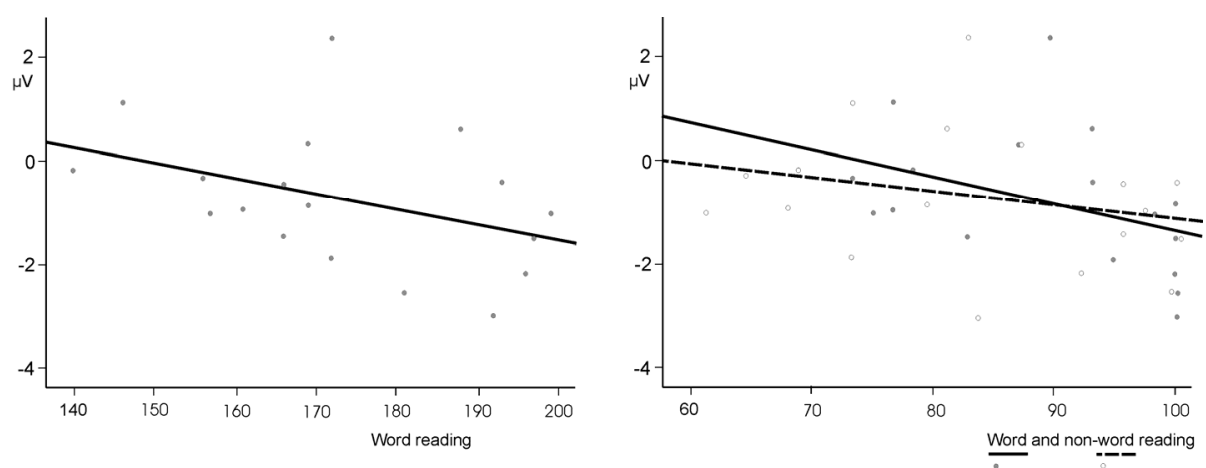

Figure 4. Correlation of the performance on a combined word/pseudoword reading task (left), and an additional word reading (right, solid line, full dots) and pseudoword reading (right, dashed line, empty dots) with the individually calculated letter-specific effect.

\section{Discussion}

The validity of the claim that the modulation of the auditory $\mathrm{MMN}$ to speech sounds found in previous cross-modal MMN studies (Froyen et al., 2009; Froyen et al., 2008) was attributed to the influence of a simultaneously presented letter was investigated. This claim is central to the interpretation of these effects as reflecting letter - speech sound integration processes. More specifically, the letter-specificity of the amplitude enhancement of the MMN to speech sounds was tested by including a condition in which a scrambled version of the same letter were presented. Additionally, all conditions were presented within subject, making it possible to calculate an individual letter-specific effect measure and to investigate its relation with reading fluency. First we investigated if the earlier reported MMN modulation was replicated with a different letter - speech sound pair. To this aim the MMN to speech sounds presented in isolation was compared with the MMN to speech sounds presented simultaneously with letters. Secondly, we tested for a letterspecific influence on the MMN by comparing the effects of the letter with the effects of the non-letter.

We replicated our earlier results and found a significant MMN amplitude enhancement if the speech sound oddball was combined with a letter. Although both letters and non-letters seemed to have an effect on the MMN amplitude to speech sound 
deviants, MMN amplitude to speech sounds presented simultaneously with letters was significantly higher in comparison with MMN amplitude to speech sounds presented with non-letters. This is a strong indication for a letter-specific, in stead of a more general, cross-modal effect of visual stimuli on speech sound processing. This interpretation of a letter-specific effect was further supported by the finding of significant correlations between the individual letter effect measures and reading fluency: higher reading fluency was clearly positively related with a higher letterspecific effect on the MMN to speech sounds. This is all the more remarkable, since the participants were all fluently reading adults without any history of reading problems. That is because it is a founding assumption of the Dual Route theory of reading that letter - speech sound processing is no longer relevant for adult fluent reading (Coltheart, Rastle, Perry, Langdon, \& Ziegler, 2001). Together, these results strongly support the previously reported effects of letters on speech sound processing and the claim that these effects reflect basic features of genuine letter - speech sound integration. The fact that we now used different letters than in the previous studies further supports this generalization. Therefore, the previous conclusion still holds: Letters and speech sounds are automatically integrated in the brain of fluently reading adults within $200 \mathrm{~ms}$ after stimulus onset (Froyen et al., 2008).

\section{Acknowledgements}

This work was partly supported by grant 608/002/2005 of the Dutch Board of Health Care Insurance (College voor Zorgverzekeringen) awarded to L.B. We thank Jeannette Boschma for recruiting participants and assistance in testing.

\section{References}

Alho, K. (1995). Cerebral Generators of mismatch negativity (MMN) and its magnetic counterpart (MMNm) elicited by sound changes. Ear \& Hearing, 16(1), 38-51.

Blau, V., van Atteveldt, N., Formisano, E., Goebel, R., \& Blomert, L. (2008). Task-irrelevant visual letters interact with the processing of speech sounds in heteromodal and unimodal cortex. European journal of neuroscience, 28(3), 500-509.

Blomert, L., \& Vaessen, A. (2009). 3DM Differential diagnostics for dyslexia: Cognitive analysis of reading and spelling. Amsterdam: Boom Test Publishers.

Boersma, P., \& Weenink, D. (2002). Praat 4.0: a system for doing phonetics with the computer. Amsterdam: Universiteit van Amsterdam.

Brus, B. T., \& Voeten, M. J. M. (1973). Een-minuuttest, vorm A en B. Nijmegen: Berkhout.

Coltheart, M., Rastle, K., Perry, C., Langdon, R., \& Ziegler, J. (2001). DRC: A dual route cascaded model of visual word recognition and reading aloud. Psychological review, 108, 204 - 256.

Ehri, L. C. (2005). Development of sight word reading: phases and findings. In M. J. Snowling \& C. Hulme (Eds.), The science of reading: A handbook (pp. 135 - 145). Oxford: Blackwell Publishing.

Frith, U. (1985). Beneath the surface of developmental dyslexia. In K. E. Patterson, J. C. Marshall \& M. Coltheart (Eds.), Surface dyslexia. London: Routledge \& Kegan-Paul. 
Froyen, D., Bonte, M., van Atteveldt, N., \& Blomert, L. (2009). The long road to automation: Neurocognitive development of letter-speech sound processing. Journal of Cognitive Neuroscience, 21, 567 580.

Froyen, D., van Atteveldt, N., Bonte, M., \& Blomert, L. (2008). Cross-modal enhancement of the MMN to speech sounds indicates early and automatic integration of letters and speech sounds. Neuroscience Letters, 430, 23-28.

Froyen, D., Willems, G., \& Blomert, L. (submitted). Evidence for a specific cross-modal binding deficit in dyslexia: An MMN-study of letter - speech sound processing.

Giard, M. H., Perrin, F, Pernier J, Bouchet P. (1990). Brain generators implicated in the processing of auditory stimulus deviance: a topographic event-related potential study. Psychophysiology, 27, 627640.

Hashimoto, R., \& Sakai, K. L. (2004). Learning letters in adulthood: direct visualization of cortical plasticity for forming a new link between orthography and phonology. Neuron, 42(2), 311-322.

Herdman, A. T., Fujioka, T., Chau, W., Ross, B., Pantev, C., \& Picton, T. W. (2006). Cortical oscillations related to processing congruent and incongruent grapheme-phoneme pairs. Neuroscience Letters, 399, 61 - 66.

Näätänen, R. (1995). The Mismatch Negativity: A powerful tool for cognitive neuroscience. Ear and Hearing, 16, 6-18.

Raij, T., Uutela, K., \& Hari, R. (2000). Audiovisual integration of letters in the human brain. Neuron, 28, 617-625.

Schröger, E. (1998). Measurement and interpretation of the mismatch negativity. Behavior Research Methods Instruments \& Computers, 30, 131-145.

Van Atteveldt, N., Formisano, E., Blomert, L., \& Goebel, R. (2007). The effect of temporal asynchrony on the multisensory integration of letters and speech sounds. Cerebral Cortex, 13, 962-974.

Van Atteveldt, N., Formisano, E., Goebel, R., \& Blomert, L. (2004). Integration of letters and speech sounds in the human brain. Neuron, 43, 271-282.

Van Atteveldt, N., Formisano, E., Goebel, R., \& Blomert, L. (2007). Top-down task effects overrule automatic multisensory responses to letter-sound pairs in auditory association cortex. Neurolmage, 36, 1345-1360. 



\section{Summary}

Written language is used to communicate in an exact manner across boundaries of place and time. In this way information and knowledge is preserved much longer than when passed on by oral communication. Thanks to written language, our knowledge of the world grows exponentially. In the present knowledge based society, reading and writing becomes crucial to obtain social and economic success. Reading and writing is not learned spontaneously. That is because the relation between letters and their corresponding speech sounds is not natural, but is based on an arbitrary consensus. Nevertheless do most children learn to read and write within a few years. About four percent of all children, however, never manage to read and/or write fast and accurately. If there is no external cause for these reading problems, for example a history of psychological problems, a low IQ or insufficient education, those children are considered to be dyslexic.

In order to obtain more insight in the process of normal and abnormal reading, it is necessary to investigate all aspects of reading. In this thesis a very basic aspect, namely the automaticity and time course of letter - speech sound processing, is investigated in adults, in normally developing readers and in dyslexic children. Neural correlates of letter - speech sound processing were measured with event related potentials (ERP), a method which records brain activity with a millisecond precision. More specifically, the mismatch negativity (MMN), a well established ERPcomponent, was used which is known to be evoked pre-attentively and without the requirement of a task.

In chapter 2, a cross-modal MMN paradigm, used in all studies presented in this thesis, is proposed as a tool to investigate automatic letter - speech sound processing with high temporal resolution. The MMN is known to reflect a specifically auditory deviance detection mechanism when in a train of standard sounds infrequently a deviant sound is presented. Experienced adult readers were presented with speech sound standards and deviants appearing either in isolation (auditory) or with a standard letter (audiovisual). Rationale was that if letters and speech sounds are automatically integrated, this should be represented in an effect of letters on the MMN to speech sounds due to a double cross-modal violation of the deviant speech sound /o/ towards the standard speech sound /a/ and the standard letter "a". To furthermore investigate the temporal window of integration, letters were presented either simultaneously or preceded the speech sounds by 100 or 200 ms. Results showed that the MMN amplitude to the speech sound deviant presented with letters was found to be enhanced in comparison with the same speech sound deviant presented in isolation, however only when letters and speech sounds were presented simultaneously. The MMN amplitude enhancement by letters diminished 
linearly with increasing stimulus onset asynchrony (SOA). Considering the early and automatic evocation of the MMN, these results strongly indicate that letters and speech sounds are early and automatically integrated in the experienced readers' brain, within a narrow temporal window of integration.

Since in the previous study (chapter 2) speech sounds were only presented either in isolation or simultaneously with letters, it cannot be excluded that merely the presence of a visual stimulus evoked the previously observed effect on the MMN amplitude. We therefore aimed at explicitly investigating the letter-specificity of the previously found cross-modal MMN amplitude enhancement. In a replication study (Chapter 6) speech sounds were presented in isolation or simultaneously with letters as well as non-letters. The non-letter was a scrambled version of the letter, and thus contained the same basic visual features as the letter but not its letter-specific content. Results revealed that in both audiovisual conditions the MMN amplitude was enhanced in comparison with the auditory only condition. However, the MMN amplitude in the audiovisual letter condition was enhanced even more in comparison with the audiovisual non-letter condition. Additionally, a correlation was found between reading fluency and the letter-specific MMN enhancement. These results clearly indicate that the previously reported letter - speech sound integration effect was genuine and, moreover, that it is strongly related with reading.

The validation of this cross-modal MMN paradigm, suited to investigate preattentive letter - speech sound processing with high temporal resolution, provided us with a tool to investigate the development of letter - speech sound processing during the first years of reading instruction (chapter 3 ). Learning the correspondences between letters and their corresponding speech sounds is considered a crucial step during reading acquisition. However, neural correlates of letter - speech sound processing in developing readers have not been reported before. Eight and eleven year old children, with respectively one and four years of reading instruction, were presented with the exact same letter - speech sound MMN paradigm as used with adults (chapter 1 ). Results reveal that in eight year old beginner readers, despite having full letter - speech sound knowledge, no early integration effect was observed. Surprisingly, even in eleven year old advanced readers this early integration effect was only present when the letter preceded the speech sound by $200 \mathrm{~ms}$ and not, like in adults, at simultaneous letter - speech sound presentation. Together, these results indicate that early and automatic letter - speech sound integration takes years to develop. Interestingly, whenever there was no early integration effect, there was an influence of letters on speech sound processing at $650 \mathrm{~ms}$ after speech sound onset. Considering the fact that, in a semi-transparent language like Dutch, children learn all letter - speech sound associations within a few months of reading instruction, it was suggested that this late effect reflects less automated letter - speech sound association. In sum, these results indicate a transition from mere association in beginner readers to more automatic, but still not "adult-like", 
integration in advanced readers. In contrast to general assumptions, the present study provides evidence for an extended development of letter - speech sound processing.

Unstable letter - speech sound associations are assumed to be the missing link between evident phonological problems and reading problems in people with developmental dyslexia. Therefore, in chapter 4, once more the same cross-modal MMN paradigm is employed to investigate letter - speech sound processing, now in a group of eleven year old dyslexics. Their results were compared with those of the advanced readers (age matched controls) as well as beginner readers (reading level controls) who participated in the developmental study (chapter 3). Remarkably, even after four years of reading instruction, children with dyslexia showed no sign of early and automatic integration. Although they showed letter - speech sound association effects in a later time window (around $650 \mathrm{~ms}$ ), this effect resembled that of the reading level controls and not that of their age matched peers. To control for letter - specificity of this late effect, we calculated correlations with behavioural reading measures. We found strong correlations of the late letter effect with word reading, somewhat lower correlations with pseudo word reading, but no correlation with rapid naming or phoneme deletion. Interestingly, the dyslexics showed very similar speech sound processing as their age matched controls when speech sounds where presented in isolation. In sum, this indicates that dyslexics do exhibit problems when processing letter - speech pairs, however, in the context of intact processing of the same speech sounds when processed in isolation. Consequently, in the present study, letter - speech sound processing problems cannot be explained by phonological problems only. We therefore advance the possibility that severe letter - speech sound processing problems may actually constitute one of the generic and proximal causes of reading problems in dyslexia.

Letter - speech sound pairs are multisensory, audiovisual objects. However, their visual and auditory properties are merely linked by an arbitrary, culturally defined consensus. This stands in contrast with for example audiovisual speech, in which visual and auditory information share time varying aspects. Recent brain imaging results have indicated that, like in audiovisual speech processing, heteromodal areas in superior temporal cortex and modality-specific auditory cortex are involved in letter - speech sound processing. Although low level visual areas are found to be involved during audiovisual speech processing as well, their roll during letter speech sound processing remains unclear. In chapter $\mathbf{5}$ the visual counterpart of the auditory mismatch negativity is used to investigate the influences of speech sounds on letter processing. Letter and non-letter deviants were infrequently presented in a train of standard letters, either in isolation or simultaneously with a speech sound. Although the findings reported in the previous chapters showed that letters systematically modulate speech sound processing, the reverse does not hold: speech sounds do not automatically influence letter processing. This asymmetric recruit- 
ment of low level sensory processing during letter - speech sound integration, contrasts with the symmetric involvement in, for example, audiovisual speech. The present results establish that low level visual processes do not constitute a basic ingredient of the cortical network responsible for the automatic integration of letters and speech sounds, probably due to the arbitrary, culturally defined link between them.

In all studies presented in this thesis a crossmodal MMN paradigm is used. Although the $\mathrm{MMN}$ is considered a purely auditory deviance detection mechanism, it has been used before to investigate neural correlates of audiovisual integration. Until now, however, these studies investigated audiovisual processing in which a deviant visual stimulus evoked an MMN by illusorily changing the percept of the standard sound. In the studies presented in this thesis the MMN amplitude enhancement reflects a more subtle crossmodal influence on speech sound processing. While a letter deviant did not evoke an auditory MMN in a series of standard speech sounds (no illusory change in auditory perception), a standard letter did influence the MMN evoked by a deviant speech sound in a series of standard speech sounds. The advantage of this crossmodal MMN paradigm is that it enables us to investigate letter speech sound processing directly, without participants having to perform a task. In this way task-related disturbing effects are avoided and it is ascertained that we do not measure, for example, the retrieval of phonological codes or the articulation of the sounds. In order to increase our insights in this crossmodal MMN, it would be interesting to investigate the exact location of the crossmodal MMN generator mechanism. It is known that deviations in different auditory attributes evoke MMNs with partially different generator mechanisms in auditory cortex. Possibly the location of the crossmodal letter - speech sound MMN partly converges with the brain areas in which $\mathrm{fMRI}$ studies revealed letter - speech sound integration effects.

To conclude, the work presented in this thesis demonstrates a tool to investigate, pre-attentively and with a high temporal resolution, letter - speech sound processing in adults, in developing readers and in dyslexics. Main conclusions are that fluent readers automatically integrate letters with speech sounds when these are presented in close temporal proximity. Secondly, development towards adult letter - speech sound processing takes multiple years and evolves from mere association into automatic integration of letter - speech sound pairs. And finally, letter - speech sound processing problems, and not (solely) phonological problems, may constitute one of the generic and proximal causes of dyslexic reading problems. 


\section{Samenvatting}

Geschreven taal laat toe om over de grenzen van de tijd heen op een exacte manier te communiceren met elkaar. Hierdoor gaat opgedane kennis minder snel verloren dan wanneer deze enkel mondeling werd doorgegeven. Dit toont zich in onze huidige kennismaatschappij die exponentieel ontwikkeld. Bijgevolg wordt lezen en schrijven steeds belangrijker om succesvol te worden in onze samenleving. Kinderen leren niet spontaan lezen en schrijven. Dat is omdat de relatie tussen letters en spraakklanken niet natuurlijk is, maar berust op een onnatuurlijke consensus. Toch ondervinden de meeste kinderen hier weinig problemen mee en kunnen zij na enkele jaren vlot lezen en schrijven. Echter, ongeveer vier procent van alle kinderen hebben het moeilijk om snel en accuraat te leren lezen en/of schrijven, zonder dat ze een lager IQ of psychologische problemen hebben of zonder dat ze minder goede scholing genieten. Hier is sprake van dyslexie.

Om meer te weten te komen over de normale en abnormale leesontwikkeling is het nuttig alle aspecten van het lezen te onderzoeken. In deze thesis heb ik me gericht op een zeer basaal aspect, namelijk hoe snel en automatisch letters aan spraakklanken gekoppeld worden in de hersenen van ervaren, minder ervaren en dyslectische lezers. Letters en spraakklanken zijn de bouwstenen van onze taal. Als het reeds misgaat in het vlot gebruik van letter - spraakklank koppelingen, heeft dat bijgevolg verstrekkende gevolgen voor de verdere leesontwikkeling.

In hoofdstuk 2 wordt een methode beschreven waarmee de snelheid en het automatisme van letter - spraakklank koppelingen kan worden onderzocht. Deze methode werd succesvol getest op een groep volwassen en dus ervaren lezers. Met behulp van event related potentials (ERP) kunnen hersensignalen gemeten worden die ontstaan na aanbieding van een bepaalde stimulus of tijdens het uitvoeren van een taak, en dit met een zeer nauwkeurige tijdsindicatie. Een welomschreven ERP component is de mismatch negativity (MMN). Deze component treedt op als er in een reeks van steeds dezelfde klanken af en toe een afwijkende klank wordt aangeboden. Het brein detecteert deze afwijking snel (binnen $250 \mathrm{~ms}$ ) en automatisch, dus zonder dat er een taak hoeft te worden uitgevoerd door de proefpersoon. In het experiment dat gepresenteerd wordt in hoofdstuk 2, werd een reeks van spraakklanken aangeboden zonder meer, of samen met de letter "a". De reeks spraakklanken bestond uit steeds dezelfde, standaard spraakklank/a/, sporadisch afgewisseld met de afwijkende spraakklank /o/. Als nu letter - spraakklank paren automatisch geïntegreerd worden in het brein (hetgeen we verwachtten bij een ervaren lezer), dan zou dit te zien moeten zijn in een effect op de MMN door de dubbele, crossmodale afwijking van de spraakklank /o/ ten opzichten van de spraakklank /a/ én de letter "a". Om ook nog na te gaan hoe groot de tijdsspanne is 
waarbinnen letters en spraakklanken automatisch worden geïntegreerd, werd de letter ofwel gelijktijdig, of 100 of 200 ms voor de spraakklanken aangeboden. Resultaten toonden dat de MMN amplitude gemeten bij letter - spraakklank paren hoger was dan wanneer gemeten bij spraakklanken alleen, maar dit was enkel het geval als de letters gelijktijdig met de spraakklanken gepresenteerd werden. Het effect op de MMN amplitude verminderde lineair wanneer de tijdsspanne tussen de letter en spraakklank aanbieding vergrootte. Gezien het bekend is dat de MMN altijd vroeg en automatisch optreedt, kunnen we uit deze resultaten afleiden dat letters en spraakspraakklanken vroeg en automatisch geïntegreerd worden in het brein van de ervaren lezer, zolang de tijdsspanne tussen beiden zeer beperkt is.

Vermits in de studie in hoofdstuk 2 spraakklanken slechts gepresenteerd werden of samen met letters of in isolatie, kon niet uitgesloten worden dat het gewoon de aanwezigheid was van een visuele stimulus waardoor er een effect gemeten werd op de MMN. Als het effect op de MMN veroorzaakt kan worden door eender welke visuele stimulus, zou dit niets zeggen over de letter - spraakklank koppelingen waarin we geïnteresseerd zijn. Er werd daarom een controle studie uitgevoerd om de letter specificiteit van de invloed van letters op de MMN te testen. In de studie beschreven in hoofdstuk 6 werden spraakklanken ofwel in isolatie ofwel gelijktijdig met letters of pseudoletters gepresenteerd. De pseudoletter was een onherkenbare versie van de letter, en had dus dezelfde basale visuele eigenschappen zonder de letter specifieke inhoud. De resultaten lieten zien dat er in beide audiovisuele condities een verhoging van de $M M N$ amplitude te zien was in vergelijking met wanneer enkel de spraakklanken aangeboden werden. Maar de MMN verhoging was groter wanneer de spraakklank samen met een letter werd aangeboden dan wanneer de spraakklank samen met de pseudoletter werd aangeboden. Verder werd er ook een correlatie gevonden tussen de letter specifieke verhoging en leesvaardigheid. Deze resultaten laten duidelijk zien dat het voorheen gevonden letter - spraakklank integratie effect echt letter specifiek is en zelfs bij ervaren lezers samenhangt met leesvaardigheid.

De validatie van deze crossmodale MMN methode, geschikt om letter - spraakklank verwerking te meten zonder de noodzakelijkheid van een taak en met een hoge tijdsresolutie, liet toe de ontwikkeling van letter - spraakklank koppelingen te onderzoeken bij schoolgaande kinderen (hoofdstuk 3). Het leren welke letters bij welke spraakklanken horen wordt verondersteld cruciaal te zijn in de leesontwikkeling. Desondanks heeft niemand ooit onderzocht hoe deze letter - spraakklank koppelingen verwerkt worden in het brein van beginnende lezers. Kinderen van acht en elf jaar oud, met respectievelijk een en vier jaar leeservaring, werden onderzocht met exact dezelfde crossmodale MMN methode als eerder beschreven in hoofdstuk 2 . De resultaten lieten zien dat er in acht jaar oude beginnende lezers geen vroege integratie effecten te zien waren, ondanks het feit dat ze op die leeftijd reeds alle letters van het alfabet aan de juiste spraakklank kunnen koppelen. Nog verrassen- 
der was de bevinding dat er in elf jaar oude gevorderde lezers wel een effect van de letter op spraakklank verwerking te zien was, echter niet wanneer beiden gelijktijdig werden aangeboden zoals bij volwassenen, maar enkel wanneer de letter 200 ms eerder kwam dan de letter. Deze twee bevindingen tonen aan dat het jaren duurt voordat letter - spraakklank verwerking zo snel en automatisch plaatsvindt als bij ervaren lezers. Verder werd er, zowel bij de beginnende als de gevorderde lezers, een interessant effect van de letter op spraakklank verwerking waargenomen later in tijd, namelijk rond $650 \mathrm{~ms}$ na stimulus aanbieding. Gezien in een semitransparante taal zoals het Nederlands (dit wil zeggen: met weinig ambigue letter - spraakklank koppelingen), de meeste kinderen alle koppelingen tussen letters en spraakklanken binnen enkele maanden kennen, werd er verondersteld dat dit late effect een soort minder geautomatiseerde letter - spraakklank associatie representeerde. Alles samen duiden deze resultaten op een overgang van associatie in beginnende lezers naar automatische, maar nog steeds niet volledig ontwikkelde, integratie bij gevorderde lezers. In tegenstelling tot eerdere aannames, lijkt de ontwikkeling naar een volwassen letter - spraakklank verwerking jarenlang te duren.

Zoals eerder aangegeven hebben moeilijkheden met lezen en schrijven een grote impact op het functioneren in onze huidige kennismaatschappij. Bijgevolg trachten we in hoofdstuk 4 om meer inzicht te verwerven in de oorzaak van dyslexie. Er wordt verondersteld dat dyslexie veroorzaakt wordt door een afwijkende spraakklankverwerking. Hierdoor zou de koppeling van spraakklanken aan letters negatief beïnvloed worden, hetgeen tot gevolg heeft dat de leesontwikkeling verstoord wordt. Hoe deze letter - spraakklank koppeling precies gebeurt in de hersenen van kinderen met dyslexie is nog niet eerder onderzocht. In hoofdstuk 4 werd er een studie uitgevoerd met exact dezelfde MMN methode als eerder beschreven (hoofdstuk 2), maar nu met een groep van elf jaar oude dyslectische kinderen. De resultaten werden vervolgens vergeleken met die van de beginnende (zelfde leesniveau) en gevorderde (zelfde leeftijd) lezers uit de ontwikkelingsstudie beschreven in hoofdstuk 3. Zelfs na vier jaar leeservaring vertoonden de dyslectische kinderen geen enkele indicatie van vroege en automatische integratie. Hoewel ze letter spraakklank associatie effecten vertoonden rond $650 \mathrm{~ms}$ na stimulus presentatie, leek dit effect op dat van de kinderen met hetzelfde leesniveau als henzelf en niet op dat van hun leeftijdsgenoten. Om na te gaan of dit latere associatie effect letter specifiek was, werden correlaties berekend met gedragsmaten van leesvaardigheid. Er werd een sterke correlatie gevonden met woord lezen, een minder sterke correlatie met pseudowoord lezen, maar geen correlatie met scores op de benoemtaken of de foneem deletie taak. Verder vertoonden dyslectische kinderen eenzelfde MMN als hun leeftijdsgenoten wanneer de spraakklanken in isolatie werden aangeboden. Dit duidt erop dat dyslectische kinderen problemen ondervinden met het verwerken van letter - spraakklank paren, terwijl het verwerken van deze spraakklanken wanneer aangeboden zonder de letters niet problematisch is. Bijgevolg 
kunnen de afwijkingen in letter - spraakklank koppelingen zoals geobserveerd in deze studie niet enkel verklaard worden door spraakklankverwerkings problemen. Daarom indiceren deze resultaten dat moeilijkheden met letter - spraakklank koppelingen, en dus niet alleen moeilijkheden met spraakklankverwerking, tot de echte oorzaken van de leesproblemen bij mensen met dyslexie behoren.

De link tussen een letter en zijn bijhorende spraakklank is gebaseerd op een onnatuurlijke, maatschappelijke afspraak. Toch zien we dat letter - spraakklank paren bij ervaren lezers automatisch geïntegreerd worden tot een audiovisueel samengesteld object (hoofdstuk 2 en 6). Andere audiovisuele stimuli, zoals audiovisuele spraak (bestaande uit lip bewegingen en spraakklanken), delen echter natuurlijke eigenschappen. De bron van een spraakklank (de mond) beweegt namelijk op een manier die samenhangt met onder andere het ritme en het volume van de spraakklank. Het is daarom interessant om na te gaan of en hoe de verwerking van letter - spraakklank paren in onze hersenen verschilt van de verwerking van andere audiovisuele stimuli. Recente functional magnetic resonance ( $\mathrm{fMRI}$ ) studies hebben aangetoond dat de hersengebieden die ingeschakeld worden bij het verwerken van letter spraakklank paren gedeeltelijk overeenkomen met de hersengebieden die actief worden bij het waarnemen van audiovisuele spraak. Er is echter nog discussie over de betrokkenheid van de visuele gebieden bij de verwerking van letter - spraakklank paren, terwijl de betrokkenheid van deze gebieden bij audiovisuele spraak verwerking algemeen aanvaard wordt. In hoofdstuk $\mathbf{5}$ werd daarom de invloed van spraakklanken op letter verwerking onderzocht met behulp van de visuele MMN. Dit is een soortgelijke ERP component als de MMN, maar dan in het visuele domein. Afwijkende letters en pseudoletters werden gepresenteerd in een stroom van standaard letters, ofwel in isolatie of gelijktijdig met spraakklanken. Hoewel de studies in alle voorgaande hoofdstukken letter specifieke effecten vonden van letters op spraakklanken, blijkt het omgekeerde niet het geval: spraakklanken beïnvloeden niet automatisch de visuele MMN veroorzaakt door letters. De asymmetrische betrokkenheid van basale hersengebieden (auditief en niet visueel) bij letter - spraakklank integratie, verschilt dus van de symmetrische betrokkenheid van basale auditieve én visuele hersengebieden zoals die terug te vinden is bij audiovisuele spraak verwerking. De vroege visuele verwerking is niet noodzakelijk betrokken bij de automatische integratie van letter - spraakklank paren, waarschijnlijk door de onnatuurlijke, maatschappelijk bepaalde relatie tussen beiden.

In deze thesis werd gebruik gemaakt van een crossmodaal MMN paradigma. Hoewel ervan uitgegaan wordt dat de MMN enkel puur auditieve verwerking representeert, werd deze component ook al eerder gebruikt om audiovisuele verwerking te onderzoeken. Maar tot nu toe werd enkel gerapporteerd dat er een MMN optrad doordat het percept van de auditieve stimulus denkbeeldig veranderde onder invloed van een afwijkende visuele stimulus. In deze thesis wordt aangetoond dat de MMN ook meer subtiele crossmodale invloeden op spraakklank verwerking kan 
representeren. Terwijl de afwijkende letter geen auditieve MMN veroorzaakte in een serie van standaard spraakklanken (dus geen denkbeeldige verandering in auditieve perceptie), werd er wel gevonden dat een standaard letter de MMN, veroorzaakt door een afwijkende spraakklank in een serie van standaard spraakklanken, kan beïnvloeden. Het voordeel van deze methode is dat het toelaat letter - spraakklank verwerking rechtstreeks te onderzoeken zonder dat proefpersonen een taak dienen uit te voeren. Om meer te weten te komen over deze crossmodale MMN zou het interessant zijn om de locatie na te gaan van het neurale generator mechanisme dat eraan ten grondslag ligt. Het is immers bekend dat afwijkingen in uiteenlopende auditieve eigenschappen MMNs veroorzaken met uiteenlopende neurale generator mechanismen in de auditieve cortex. Mogelijk komt de locatie van de crossmodale MMN overeen met de hersengebieden waar eerdere fMRI studies letter - spraakklank integratie effecten hebben gevonden.

Ter conclusie: de studies die in deze thesis werden gepresenteerd demonstreren een methode om letter - spraakklank koppelingen te onderzoeken in de hersenen van ervaren en beginnende lezers alsook dyslectici. Een groot voordeel van deze methode is dat er met een hoge tijdsresolutie wordt gemeten en dat het niet nodig is een taak op te leggen aan de proefpersonen. Op die manier worden taak gerelateerde effecten uitgesloten, zodat we er zeker van kunnen zijn dat we niet het ophalen van de spraakklank of het uitspreken ervan aan het meten zijn. De belangrijkste conclusies zijn dat ervaren lezers vroeg en automatisch letter - spraakklank paren integreren wanneer ze gelijktijdig worden aangeboden. Ten tweede, de ontwikkeling tot een volwassen letter - spraakklank verwerking duurt ettelijke jaren en evolueert van associatie naar automatische integratie van letter - spraakklank paren. Ten derde, problemen met de verwerking van letter - spraakklank paren, en niet (enkel) problemen met spraakklank verwerking lijken ten grondslag te liggen aan leesproblemen van mensen met dyslexie. 


\section{Acknowledgements}

While thinking about my PhD-studentship, I realize that I had the opportunity to work in an extraordinary environment in which I met a lot of nice colleagues and made some real friends. I feel lucky to have been able to do a PhD here in Maastricht at the faculty of Psychology and Neuroscience. For creating this opportunity I have to thank my promoter Leo; thanks for your trust in me and all your contributions to this thesis.

Nienke and Milene helped me a lot with my first steps in research. Like two older sisters, they took me by the hand and contributed enormously by advising me how to analyse data, by brainstorming together with Leo en me about results and by reading and commenting each new version of the papers that we have published together. Thanks a lot for everything you two taught me!

Already during my internship, I sensed the special atmosphere at Maastricht University that I have enjoyed a lot as an employee. Although most colleagues are very busy, they are usually open for a brainstorm, helping out or a friendly chat. Seniors like Rainer, Peter, Elia, Alex, Lisa, Arie and Bernadette contribute a lot to this atmosphere. I want to thank you all for inspiring me.

I want to thank all my colleagues for the nice moments both at and after work. I have to mention some people by name: Teresa (those famous lunches, where have they gone!), Federico (for your eternal hospitality), Lars R (roommate at HBM Florence), Aline and Marin (for organizing numerous social events), Bettina (always helpful), Martin (for editing a paper and great humour), Maria (for experiencing Helsinki and numerous brainstorm sessions). While writing this I realize that I want to mention many more people, so to keep it short: Tank you all for a great working atmosphere!

During my PhD I experienced a lot of support from Annemie and Rini from the secretary office and from Jacco and Johan for technical support in the EEG labs. I furthermore want to thank the staff of primary school St. Aloysius and De Perroen as well as the Regional Institute for Dyslexia for their help in collecting subjects and of course all the children who participated in the experiments.

While thinking about with whom I've shared the room at work, I notice it is quiet a list (I hope that it's not because of me that there was such a turnover!): Hanne, Kim, Jochen, Laura, Denise, Anke and Felix. Thanks for your company and the nice times 
we have shared. I want to thank Anke and especially Hanne, for their contributions to the work presented in this thesis.

During my first years in Maastricht I rented a beautiful house together with Pim and Vera. With pleasure I think back at the parties, the summer evenings at our rooftop terrace, the exotic cooking and the warm coming home after work to you guys. Vera, I admire you for all your knowledge and your drive, but most of all I appreciate your friendly, trustworthy personality. Thanks for being my paranimph.

During the last years I have had many opportunities to travel abroad. Due to the nice colleagues I travelled with, those trips were each time an extremely motivating and joyful experience. I will never forget our road trip trough California in a camper: Anniek (you still surprise me), Petra (how much talent fits in one little person?), Noël (eitnieneoiemotmeiothio!!!), Vera and Gonny. Remember: What happened in California stays in California! Jeannette, although you were not with us on the trip, it feels like you are part of this group. Moreover I want to thank you for your contributions to the last chapter of the thesis. Gonny, unfortunately all the gossip about us is untrue. I admire you for your perseverance (CPE!) and ability to get back on your feet and make the best of every situation. Thanks for your great company and lovely chats about life. Thanks for being my paranimph.

I feel lucky to have been able to stay in touch with many friends from Genk (Jan, Tom, Roeland, Wouter, Michiel,...) and from my study time in Leuven (Anke, Annelies, Nele, Goedele en Lizzy). Thanks for your interest and accepting my slight change in accent.

Pieter, thank you for designing such a cool cover for my thesis. Since we were old enough to play together, we have enjoyed each others fantasy. This cover is again an example of how our creative thoughts come together and create a wonderful world. Thank you Pieter and Joke for one of the most impressive experiences in my life, which is giving me the wonderful honour of being the godfather of your beautiful daughter Stien.

Moeke and Vake, you are the best parents ever, seriously! I appreciate you more and more as two wonderful people with whom I would love to share many more of those beautiful moments as we have done in the past. You taught me the best lesson there is to be taught, mainly by showing how it's done: enjoy life.

Marloes, you are my girl. I love you. Thanks for being there for me, with your particular humour and advice. Thanks for all your patience and support. Now it's time to celebrate, let's dance, you and me! 


\section{Curriculum Vitae}

Dries Froyen was born in Genk (Belgium) on the fourth of April 1980. He studied Classical languages at the Sint Jan Berchmans College in Genk from 1992 until 1998. He attended one year at the Vidergoande scole in Molde (Norway) as an AFS exchange student. From 1999 until 2004 he studied Psychologische wetenschappen at the Katholieke Universiteit Leuven (Belgium). The last three years he specialized in theoretical psychology, more specific Behavioural-Neuroscience. In his Master thesis, under supervision of Prof. Dr. Johan Wagemans, he used event related fMRIadaptation to investigate the involvement of low level visual areas in object recognition. The last year of his study he went with ERASMUS to Maastricht University (The Netherlands), were he did a research internship about letter - speech sound processing under supervision of Prof. Dr. Leo Blomert at the faculty of Psychology and Neuroscience. He graduated in the summer of 2004. From the autumn 2004 until the winter 2005 he was a research assistant at the faculty of Psychology and Neuroscience, were he continued his work on letter - speech sound processing. Besides this, studies about allophonic perception were set up in cooperation with Dr. Willy Serniclaes at the CNRS in Paris (France). In the spring of 2005 he started his PhD at Maastricht University, faculty of psychology and neuroscience, under supervision of Prof. Dr. Leo Blomert. During his PhD he conducted the experiments presented in this thesis. Since the summer of 2009 he continues his work as a post-doc researcher at Maastricht University, faculty of psychology and neuroscience, under supervision of Prof. Dr. Leo Blomert.

\section{Contact information}

Dries Froyen

President Rooseveltlaan $141 \mathrm{C}$

6224 CJ, Maastricht

0031647183132

0032474938477

d.froyen@maastrichtuniversity.nl 